\title{
Rastreador linear quadrático com custo médio de longo prazo para sistemas lineares com saltos markovianos
}

\author{
Luiz Henrique Barchi Bertolucci
}

Orientador: Prof. Dr. Eduardo Fontoura Costa

Dissertação apresentada ao Instituto de Ciências Matemáticas e de Computação - ICMC-USP, como parte dos requisitos para obtenção do título de Mestre em Ciências - Ciências de Computação e Matemática Computacional. VERSÃO REVISADA.

USP - São Carlos

Junho/2011 
Ficha catalográfica elaborada pela Biblioteca Prof. Achille Bassi e Seção Técnica de Informática, ICMC/USP, com os dados fornecidos pelo(a) autor(a)

Bertolucci, Luiz Henrique Barchi

B546r Rastreador linear quadrático com custo médio de longo prazo para sistemas lineares com saltos markovianos / Luiz Henrique Barchi Bertolucci; orientador Eduardo Fontoura Costa -- São Carlos, 2011 . $83 \mathrm{p}$.

Dissertação (Mestrado - Programa de Pós-Graduação en Ciências de Computação e Matemática Computacional) -Instituto de Ciências Matemáticas e de Computação, Universidade de São Paulo, 2011.

1. Rastreador de alvos. 2. Sistemas lineares. 3. Processo markoviano. 4. Planejamento hidrotérmico. I. Costa, Eduardo Fontoura, orient. II. Título. 
Dedico este trabalho aos meus pais, Cesar e Alice. 



\section{Agradecimentos}

Não preencho este espaço apenas por me ser permitido pela ABNT. Aqui, sinto vontade de agradecer a todos os que contribuiram para minha formação. Mas, seria impossível lista-los! Muita gente que nem ao menos conheço! Assim, quero que todos se sintam representados por estes nomes aqui levantados. Agradeço em primeiro lugar todo o amor e a imensa paciência que meu pai Cesar, minha mãe Alice e minhas irmãs, Lidia e Maria, me ofereceram incondicionalmente por toda as nossas vidas! Agradeço também minha namorada Luciana por toda carinho, compreensão e ótimos conselhos durante estes anos. Ao Eduardo por toda confiança, dedicação e paciência para assuntos que extrapolaram os temas deste trabalho. Agradeço ainda ao CNPq pela bolsa concedida e as várias outras pessoas que também contribuiram muito para o desenvolvimento deste trabalho, em ordem alfabética, cito Atilio Aguilera, Ieda Hidalgo, João Brandão, tia Leninha, Luana Paiva, Marinho Andrade e Rodrigo Ramos. Por fim, agradeço também o apoio do cunhado Faustinho, tio José, tia Silvia, além, é claro, dos inúmeros amigos e amigas de todas as repúblicas e de todas as vidas. A todos vocês, mestres em minha vida, meus sinceros agradecimentos! 

Neste trabalho estudamos um controlador denominado rastreador linear quadrático (RLQ) com custo médio de longo prazo (CMLP) para sistemas lineares com saltos markovianos (SLSM). Mostramos que o conceito de detetabilidade uniforme, juntamente com a hipótese de que o regulador linear quadrático associado ao RLQ tenha custo uniformemente limitado, são suficientes para que o controle obtido seja estabilizante em um certo sentido. A partir deste resultado, e considerando as mesmas hipóteses, demonstramos a existência do CMLP. Com isto, estendemos os resultados dispostos na literatura desde que consideramos um sistema variante no tempo e uma estrutura mais geral para a cadeia de Markov. Além disto, avaliamos a aplicação deste controlador no planejamento da operação de um sistema hidrotérmico. Para isto, utilizamos o sistema de usinas do rio São Francisco, em dois casos de estudo, para comparar o desempenho do controlador estudado em relação à solução ótima para o problema, encontrada com o uso da programação dinâmica estocástica, e em relação à solução obtida via programação dinâmica determinística. Os resultados sugerem que o RLQ pode representar uma alternativa interessante para o problema de planejamento hidrotérmico. 

In the present work we study the reference tracking controller (RTC) for the long run average cost (LRAC) problem for Markov jump linear systems. We show that uniform detectability and an hypothesis that the linear quadratic regulator associated with the RTC has uniformly bounded cost, together, are sufficient conditions for the obtained control be exponentially stabilizing in a certain sense. This result allows us to demonstrate the existence of the LTAC under the same hypotheses. The results can be regarded as an extension of previous works, since we have considered a more general framework with time-varying systems and quite general Markov chains. As an applicatioin, we consider the operational planning of hydrothermal systems. We have considered some power plants of the São Francisco river, in two different scenarios, and we have compared the performances of the RTC and standard controls obtained by deterministic and stochastic dynamic programming, indicating that the RTC may be an interesting alternative for the hydrothermal planning problem. 

1 Introdução 1

1.1 Considerações Gerais . . . . . . . . . . . . . . . . . 1

1.2 RLQ com CMLP para SLSM . . . . . . . . . . . . . . 2

1.3 Aplicação no Planejamento da Operação de um Sistema Hidrotérmico . 5

2 RLQ com CMLP sujeito a um SLSM $\quad 9$

2.1 Notações e Operadores . . . . . . . . . . . . . . . . . . 9

2.2 Definição do Problema . . . . . . . . . . . . . . . . . 11

2.3 Estabilidade Exponencial do Regulador Linear Quadrático . . . . . . . 15

2.4 Existência do CMLP . . . . . . . . . . . . . . . . . . 20

3 Planejamento da Operação de um Sistema Hidrotérmico 25

3.1 Definição do Problema . . . . . . . . . . . . . . . 25

3.2 Métodos e Casos de Estudos . . . . . . . . . . . . . . . . . . 29

3.2.1 Simulações . . . . . . . . . . . . . . . . . 32

3.2.2 Soluções para $\mathbf{P}_{\mathbf{d}}^{\mathcal{H}}, \check{\mathbf{P}}_{\mathbf{d}}^{\mathcal{H}}, \mathbf{P}_{\mathbf{v}}^{\mathcal{H}}$ e $\check{\mathbf{P}}_{\mathbf{v}}^{\mathcal{H}} \ldots \ldots . \ldots . \ldots . \ldots . \ldots 33$

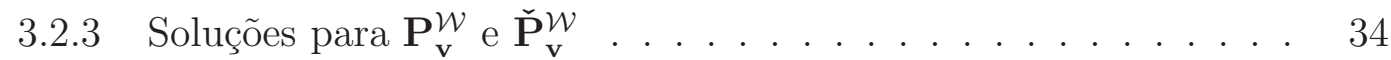

3.3 Resultados e Discussão . . . . . . . . . . . . . . . . . . 37

$\begin{array}{lll}4 \text { Conclusões } & 47\end{array}$

A Resultados auxiliares para RLQ com CHF 53

A.1 Prova para o RLQ com CHF para SLSM . . . . . . . . . . . . . . 53

A.2 Expressões Alternativas para o $\mathrm{CHF}$. . . . . . . . . . . . . . 59 
$\begin{array}{ll}\text { B Planejamento Hidrotérmico } & 61\end{array}$

B.1 Construção do Modelo . . . . . . . . . . . . . . . . . . 61

B.2 Dados dos Reservatórios . . . . . . . . . . . . . . . 63

B.3 Modelo Markoviano para a Vazão Afluente de Sobradinho . . . . . . . . 65

B.4 Demais Resultados - Figuras . . . . . . . . . . . . . . 67 


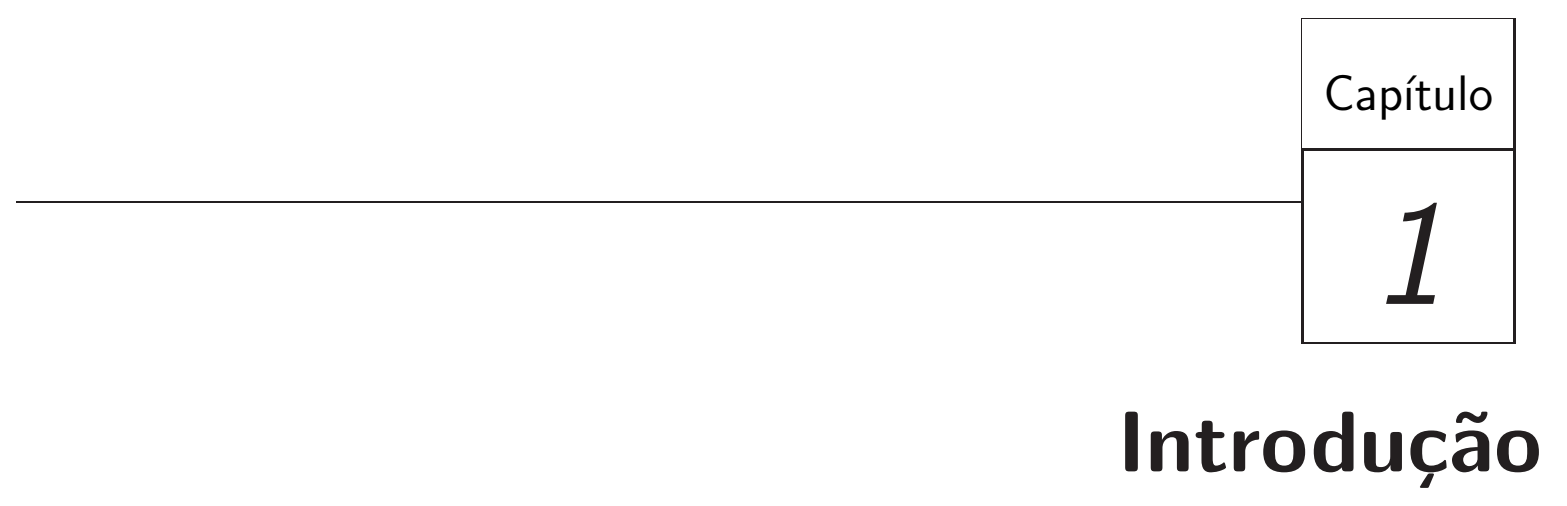

\subsection{Considerações Gerais}

O principal objetivo da área de controle ótimo é operar um sistema dinâmico enquanto minimiza um certo funcional de custo. Se este sistema dinâmico está sujeito a mudanças abruptas que seguem um processo estocástico markoviano e pode ser representado por uma equação linear, então pertence à classe dos sistemas lineares com saltos markovianos (SLSM). Neste trabalho estudamos o controlador denominado rastreador linear quadrático (RLQ) aplicado em um SLSM a tempo discreto. Os RLQ são projetados para aproximar os valores do estado e o próprio controle à valores pré-estabelecidos, denominados alvos ou curvas guia. Nosso principal objetivo neste estudo foi apresentar condições suficientes para a existência de um custo com horizonte de tempo infinito associado ao RLQ, denominado custo médio de longo prazo (CMLP). Além disto, mostramos que estas condições são também suficientes para a estabilidade do SLSM em um

certo sentido. É importante mencionar que assumimos parâmetros variantes no tempo e definimos a cadeia de Markov como finita e não homogênea no tempo, ou seja, não assumimos ergodicidade para cadeia. Os detalhes deste desenvolvimento estão dispostos na seção seguinte e no Capítulo 2.

Um tópico de estudo secundário deste trabalho consistiu em aplicar o RLQ com CMLP no problema de planejamento de longo prazo da operação de um sistema hidro- 
térmico. A solução para o problema de planejamento hidrotérmico consiste em definir regras para a turbinagem de um sistema de usinas hidroelétricas que promovam o menor custo de complementação energética por termoelétricas. Os rastreadores de alvo são importantes neste contexto, principalmente considerando o múltiplo uso da água [28]. A estratégia é aproximar o volume armazenado e a turbinagem dos reservatórios à valores pré-definidos que, além de produzir uma quantidade de potência elétrica satisfatória, mantenham boas condições de navegação, qualidade de água e proteção contra inundações na bacia, por exemplo. Além disto, como apontado por [6], é aconselhável adotar custos na forma de CMLP para o gerenciamento de reservatórios, principalmente considerando o uso da água como fator chave em um ecossistema. Os únicos trabalhos que utilizaram o RLQ neste contexto são [34, 19, 23]. Nestes, os autores empregaram o RLQ para o controle a curto prazo de um ou mais reservatórios com finalidades de gerar energia ou promover o controle de cheias. Apesar de relatarem bons resultados, estes trabalhos não apresentam comparações do desempenho do RLQ frente a outras técnicas disponíveis para o problema. Assim, neste trabalho pretendemos tratar desta lacuna na medida que empregamos o RLQ no planejamento de longo prazo e comparamos seus resultados com os obtidos via programação dinâmica estocástica (PDE) e determinística (PDD). O objetivo desta vertente de pesquisa foi mostrar que este controlador, apesar de resolver um problema simplificado, é uma alternativa interessante para o controle de sistemas hidrotérmicos com múltiplos reservatórios. Os detalhes desta implementação estão apresentados na seção final desta Introdução, bem como no Capítulo 3.

\subsection{RLQ com CMLP para SLSM}

Os SLSM podem ser utilizados como modelo para uma grande variedade de sistemas, dos quais destacamos: receptores térmicos solares [31], modelos macroeconômicos [14], sistemas robóticos [27], processamentos de sinais [15], gerenciamento de carteira [11], controle de despacho de energia [32], dentre outros. Estes sistemas vêm sendo estudados há pelo menos trinta anos, de modo que boa parte dos resultados condizentes aos sistemas lineares clássicos, já possuem equivalentes para o caso com saltos [26]. Como exemplo de um SLSM, considere a seguinte dinâmica que trataremos neste trabalho,

$$
x(t+1)=A_{\theta(t)}(t) x(t)+B_{\theta(t)}(t) u(t)+e_{\theta(t)}(t)+H_{\theta(t)}(t) \nu(t), t \geq 0,
$$


$\operatorname{com} x(0)=x_{0}$ e $\theta(0)=\theta_{0}$ dados. Nesta equação, o par $\{x(t) ; \theta(t)\}$ é definido como o estado do sistema no instante $t, u(t)$ é a variável de controle, $\nu(t)$ é um ruido aditivo e o termo $e_{\theta(t)}(t)$ representa uma entrada exógena conhecida. Observe que os parâmetros $A(t), B(t), H(t)$ e $e(t)$ estão condicionados aos valores de $\theta(t)$, definido como o estado de uma cadeia de Markov conhecida. Neste trabalho assumimos observação completa do estado de (1.1), ou seja, as variáveis $x(t)$ e $\theta(t)$ podem ser observadas em todo instante $t$.

Para projetar o controlador ótimo de um SLSM, devemos definir um funcional de custo, também denominado critério de desempenho, que representa uma avaliação da trajetória do estado e, possivelmente, dos valores de $u(t)$. Assim, consideramos um funcional de custo dado por

$$
\begin{aligned}
z(t)=(x(t) & \left.-\bar{x}_{\theta(t)}(t)\right) Q_{\theta(t)}(t)\left(x(t)-\bar{x}_{\theta(t)}(t)\right) \\
& +\left(u(t)-\bar{u}_{\theta(t)}(t)\right) R_{\theta(t)}(t)\left(u(t)-\bar{u}_{\theta(t)}(t)\right),
\end{aligned}
$$

sendo $\bar{x}_{\theta(t)}(t)$ e $\bar{u}_{\theta(t)}(t)$, nesta ordem, os alvos pré-estabelecidos para o estado e controle, e $Q_{\theta(t)}(t)$ e $R_{\theta(t)}(t)$ matrizes de ponderação, ou também denominadas matrizes de peso, pré-definidas. Note que o índice $z(t)$ pode ser compreendido como uma medida do afastamento das variáveis $x(t)$ e $u(t)$ em relação aos seus respectivos alvos. Neste trabalho, denominamos como RLQ um controlador que procura minimizar um funcional de custo baseado neste índice. Observe que fazendo $\bar{x}_{\theta(t)}(t)=0$ e $\bar{u}_{\theta(t)}(t)=0$ para todo $t \geq 0$ e assumindo (1.1), obtemos um funcional de custo referente ao regulador linear quadrático, um problema bem conhecido na área de controle e que desempenha um papel importante neste trabalho.

De acordo com o horizonte de tempo considerado, podemos definir dois funcionais de custo baseados em $z(t)$. Para um horizonte finito, consideramos

$$
\mathcal{W}_{0}^{T}=E\left\{\sum_{t=0}^{T} z(t)\right\}
$$

denominado custo de horizonte finito ( $\mathrm{CHF})$, em que $T$ é o horizonte de tempo e $E\{$. a representação do valor esperado. O controlador que minimiza (1.3) para quando se assume observação completa do estado do SLSM, está bem descrito na literatura e pode ser obtido em [14]. Dada sua importância neste trabalho, apresentamos estes resultados em detalhes na Seção 2.2, bem como uma versão da prova para o controle ótimo na Seção A.1. 
Devido ao ruido aditivo e à estocasticidade dos alvos, é comum obtermos $\mathcal{W}_{0}^{T} \rightarrow \infty$ para um horizonte de tempo infinito. Por isto, neste caso considera-se geralmente o custo na forma de CMLP, como o seguinte

$$
\mathcal{W}=\lim \sup _{T \rightarrow \infty} \frac{\mathcal{W}_{0}^{T}}{T+1}
$$

Este funcional foi utilizado em [10], em que os autores mostram a existência do CMLP definido para o regulador linear quadrático sujeito a um SLSM, assumindo entrada exógena nula em (1.1). Em [26], os autores generalizam estes resultados ao considerarem $\bar{x}_{\theta(t)}(t)=\bar{x}$, para todo $t \geq 0$. Em ambos os casos, os autores mostram a existência de $\mathcal{W}$ em contextos com cadeia ergódica e sistemas invariantes no tempo.

Diante deste contexto, pretendemos neste trabalho estudar o RLQ com CMLP para SLSM em um cenário mais amplo que os abordados em [10, 26]. Assim, consideramos os parâmetros variantes no tempo, como em (1.1), e alvos também submetidos a saltos como podemos observar em (1.2). Além disto, assumimos que a cadeia de Markov seja apenas finita, o que inclui cadeias não-ergódicas, periódicas e etc. Uma das principais contribuições foi mostrar que, o conceito de detetabilidade uniforme [1,9] juntamente com a hipótese de que o regulador linear quadrático associado ao problema tenha custo uniformemente limitado, são suficientes para que (1.4) esteja bem definido e para que o controle obtido seja estabilizante num certo sentido.

Os resultados deste trabalho podem ser compreendidos como uma generalização de [10, Theorem 4.6], uma vez que naquele texto é considerado ergodicidade para a cadeia de Markov e existência de uma solução estabilizante para Riccati, ou seja, sistema MS-Estabilizável. De fato, a hipótese de existência de um limitante para o custo do regulador linear quadrático é garantida no caso particular em que há ergodicidade, invariância dos parâmetros e o sistema é MS-estabilizável. Detetabilidade também é necessária em [10] para assegurar que a solução estabilizante da Riccati corresponda à solução mínima do problema de controle. Em relação a [26], podemos fazer comparações semelhantes, desde que os autores consideraram a existência e unicidade das soluções de Riccati no contexto de sistemas invariantes e ergódicos que, similarmente ao exposto acima, implica nas mesmas condições consideradas neste trabalho. 


\subsection{Aplicação no Planejamento da Operação de um Sistema Hidrotérmico}

O problema do planejamento da operação de um conjunto de usinas hidroelétricas visa estabelecer regras que determinem a turbinagem de cada reservatório de acordo com o volume de água presente no sistema e com os valores observados para as vazões afluentes. Esta é uma tarefa de alta complexidade, uma vez que envolve inúmeras restrições, relacionadas à capacidade de armazenamento e turbinagem dos reservatórios; dependência de variáveis estocásticas, considerando principalmente a aleatoriedade das vazões afluentes; e utilização de equações não lineares, como, por exemplo, para o cálculo da potência gerada pelas usinas. A dificuldade torna-se ainda maior quando consideramos o múltiplo uso da água, que inclui, a navegação, recreação, aquicultura; e as diversas funções de um sistema de barragens, como por exemplo, a geração de energia, o controle de cheias na bacia e a manutenção da qualidade da água. Durante os últimos 50 anos vários métodos foram propostos para tratar deste problema, dos quais destacamos a programação linear, programação não linear e a programação dinâmica $[37,18]$.

Podemos categorizar este problema em curto, médio e longo prazo [24], de acordo com o horizonte de tempo considerado. Para o planejamento de longo prazo, adota-se em geral um modelo obtido via balanço hídrico com discretização mensal [18]. Neste trabalho, assim como em $[17,3,2,33,20,16]$ e muitos outros, utilizamos uma cadeia de Markov para representar o comportamento estocástico da vazão afluente. Com isto, para um sistema com $N$ reservatórios, é possível obter o seguinte modelo,

$$
x(t+1)=A(t) x(t)+B(t) u(t)+e_{\theta(t)}(t)+C(t) s(t)
$$

com $x(0)=x_{0} \in \mathbb{R}^{N}$ dado, sendo $x(t) \in \mathbb{R}^{N}$ o vetor de estado contínuo, que denominaremos simplesmente de vetor de estados, cujo componente $[x(t)]_{i}$ representa o volume armazenado de água no reservatório $i$ no início do $t$-ésimo intervalo de tempo, $u(t) \in \mathbb{R}^{M}$ o vetor de controle, em que $[u(t)]_{i}$ indica a turbinagem de água para o mesmo reservatório durante o intervalo de tempo $t, e_{\theta(t)}(t) \in \mathbb{R}^{N}$ o vetor de entradas exógenas que representa, conjuntamente, a vazão incremental dos reservatórios e perdas, tais como, por usos consuntivos e pelo fenômeno evaporação, e $s(t)$ a função que determina o vertimento dos reservatórios. As matrizes $A(t), B(t)$ e $C(t)$ se relacionam com a rota d'água dentro do sistema e a evaporação, quando este fenômeno não é desprezível. A variável $\theta$ representa o estado da vazão incremental e é dada por uma cadeia 
Capítulo 1 - Introdução

de Markov conhecida. À equação anterior devemos ainda impor restrições às variáveis $x(t)$ e $u(t)$, referentes aos limites mínimos e máximos de armazenagem e turbinagem. Observe que neste modelo desprezamos o tempo de deslocamento do volume d'água entre os reservatórios, como em geral adotado para esta escala de tempo [3].

Para a otimização do problema, podemos adotar alguma das diversas funções objetivo sugeridas na literatura, tais como, minimização da perda de energia potencial armazenada, maximização da energia total produzida, minimização da quantidade de água vertida, dentre outras [35, 37]. Neste trabalho consideramos uma função objetivo relacionada com a minimização dos custos de produção de energia em um sistema hidrotérmico $[24,30]$. A produção de energia hidroelétrica, além de menos poluente, apresenta um custo muitas vezes menor que a termoelétrica. Assim, uma boa estratégia é utilizar prioritariamente hidroelétricas, enquanto termoelétricas devem cumprir um papel de complementação para garantir o atendimento à demanda energética. No Brasil, com a criação do Sistema Interligado Nacional (SIN) facilitou-se o intercâmbio de energia elétrica entre as regiões geográficas brasileiras, favorecendo a implementação desta estratégia, comumente denominada minimização da energia complementar [4]. Com isto, passamos a denominar este problema como planejamento da operação de um sistema hidrotérmico, cujo objetivo é a minimização do seguinte custo,

$$
\mathcal{H}_{u}^{T}\left(x_{0}\right)=\sum_{t=0}^{T}[D(t)-P(t)]^{2},
$$

sujeito a (1.5), em que $T$ é o horizonte de planejamento, $D(t)$ é a demanda de potência a ser atendida no período $t$ e $P(t)$ é a potência total gerada pelo sistema hidrotérmico. Neste trabalho, utilizamos esta equação para, dentre outros, avaliar o desempenho dos controladores e obter os alvos e matrizes de peso para o RLQ.

A principal vantagem da aplicação do RLQ é a possibilidade de se obter soluções analíticas para o problema. Isto significa que as variáveis $x(t)$ e $u(t)$ não precisam ser discretizadas, de modo que o método não sofre do denominado "mal da dimensionalidade", como ocorre com a programação dinâmica [37]. Como consequência, podemos considerar modelos mais complexos, ou realistas, para a dinâmica da vazão afluente ao incluirmos correlações temporais ou espaciais, como mencionado por [19]. Por outro lado, podemos apontar a falta de garantia quanto à obtenção da solução ótima no sentido de (1.6), como a principal desvantagem na utilização desta técnicas no planejamento hidroelétrico. Além de utilizar uma função objetiva que não reflete explicitamente o custo do problema, o modelo linear utilizado pelo RLQ, equação (1.1), não admite a inclusão do termo não linear $s(t)$, como em (1.5), e não contempla as demais 
restrições para as variáveis de volume armazenado e turbinagem, o que pode levar a soluções impraticáveis e à necessidade de aproximá-las para ações factíveis durante a operação, conduzindo, invariavelmente, a soluções sub-ótimas [6].

Outras dificuldades se referem à obtenção das matrizes de ponderação $Q$ e $R$, e dos alvos $\bar{x}(t)$ e $\bar{u}(t)$. Encontrar tais matrizes e vetores de modo a obter boas soluções para os problemas que envolvem geração hidroelétrica pode ser uma tarefa tão complexa quanto resolvê-lo em sua forma original [6]. Para contornar estas dificuldades, propomos neste trabalho utilizar a solução de um cenário mais simples, em que as vazões são determinísticas. A idéia básica é induzir a minimização do custo (1.6) pelo RLQ com o uso da solução obtida via programação dinâmica determinística (PDD). Para isto, como sugerido por [18], utilizamos esta solução para encontrar as curvas guia para o rastreador de alvos. Além disto, apresentamos um método que utiliza também a PDD para obter as matrizes de ponderação para o RLQ.

Devido às desvantagens apontadas, a aplicação do RLQ para o controle de reservatórios tem sido pouco motivada, principalmente considerando o planejamento da operação de longo prazo. O primeiro trabalho a considerar o RLQ no gerenciamento de reservatórios foi [34]. Neste, a função dos reservatórios era fornecer proteção contra inundações na bacia. Apesar dos autores apresentarem bons resultados para o controle em tempo real, não apresentaram um método para obter a curva guia e as matrizes de ponderação. Já em [19], os autores apresentaram a primeira aplicação do RLQ no planejamento da geração hidroelétrica, para o controle em tempo real de dois reservatórios. As matrizes de peso foram obtidas diretamente da função de custo a partir de uma aproximação de Taylor de primeira ordem. Para encontrar os alvos, os autores empregaram a solução do problema para um cenário determinístico, similar ao procedimento que utilizamos neste trabalho. Em [23], os autores mostraram que a operação diária real de um reservatório pode ser bem aproximada pela solução obtida a partir de um controlador RLQ. Para isto, a partir dos dados de operação real de um certo reservatório, encontraram as matrizes de peso via minimização do erro quadrático em um problema de identificação do modelo. Apesar dos trabalhos citados acima relatarem bons resultados, nenhum destes compararam desempenho do RLQ com técnicas alternativas ou utilizaram-no no planejamento de longo prazo de um sistema hidroelétrico.

Diante disto, o objetivo deste trabalho foi mostrar que o RLQ pode produzir resultados satisfatórios para o planejamento de longo prazo de um sistema com múltiplos reservatórios. Para isto, comparamos, além de outras variáveis de interesse, os custos hidrotérmicos do RLQ com os custos obtidos por PDE e com o uso da PDD. É 
importante apresentar os custos relativos à PDE pois, desconsiderando a necessária discretização das variáveis, a solução dada por esta técnica pode ser considerada como ótima para o problema [22]. Por outro lado, é também interessante utilizar a PDD como técnica comparativa uma vez que, por ser menos suscetível ao "mal da dimensionalidade", esta é também bastante empregada neste contexto. Para avaliar o desempenho do RLQ, utilizamos as usinas do rio São Francisco em dois casos de estudo que, por suas características, fazem com que as restrições de volume armazenado sejam ativadas de maneira distintas, levando a resultados claramente contrastantes. Para estas comparações, utilizamos um conjunto de séries sintéticas de vazões incrementais, obtidas via simulação de Monte Carlo, como também a série histórica real de vazões. 


$\frac{-4}{2}$

\section{RLQ com CMLP sujeito a um SLSM}

Neste capítulo, mostraremos a existência do CMLP para o RLQ sujeito a um SLSM. Para isto, assumimos que o sistema atende ao conceito de detetabilidade uniforme e que o custo do regulador linear quadrático, associado a este mesmo sistema, seja uniformemente limitado. A idéia básica foi aplicar o controlador de [14] em um horizonte de tempo infinito e mostrar que, nas condições propostas, o CMLP é limitado. Diante disto, apresentaremos na Seção 2.1 algumas notações e operadores básicos que serão utilizados neste desenvolvimento. Após, na Seção 2.2, definiremos o problema a ser tratado e também os conceitos de detetabilidade uniforme e custo uniformemente limitado, além da definição de estabilidade exponencial empregada. Mostraremos ainda nesta seção a solução para o RLQ com CHF, obtido de [14]. Na Seção 2.3, será apresentado essencialmente um resultado relacionado com a estabilidade do sistema. Finalmente, na Seção 2.4, apresentaremos o principal resultado deste capítulo, a prova da existência do CMLP.

\subsection{Notações e Operadores}

Seja $\mathcal{O}:=\{1, \ldots, \eta\}$ um conjunto finito e $\mathcal{M}^{n, s}$ o espaço linear formado por famílias de $\eta$ matrizes reais pertencentes ao espaço $\mathbb{R}^{n, s}$, ou seja, $\mathcal{M}^{n, s}=\left\{U=\left(U_{1}, \ldots, U_{\eta}\right)\right.$ : $\left.U_{i} \in \mathbb{R}^{n, s}, i \in \mathcal{O}\right\}$. Considere as operações matemáticas envolvendo elementos de $\mathcal{M}^{n, s}$ 
definidas matriz a matriz. Como ilustração, para $U, V \in \mathcal{M}^{n, s}$, temos $U+V=\left\{U_{1}+\right.$ $\left.V_{1}, \ldots, U_{\eta}+V_{\eta}\right\}$. Seja $\mathcal{M}^{n}$ a representação do subespaço linear de $\mathcal{M}^{n, n}$ constituído por matrizes simétricas. Considere também $\mathcal{M}^{n 0}\left(\mathcal{M}^{n+}\right)$ o cone fechado (aberto) de matrizes semi-definidas (definidas) positivas de $\mathcal{M}^{n}$. Considere a norma 2 definida como

$$
\|U\| \triangleq\left(\sum_{i \in \mathcal{O}} \operatorname{tr}\left\{U_{i}^{\prime} U_{i}\right\}\right)^{\frac{1}{2}},
$$

para $U \in \mathcal{M}^{n, s}$, sendo $\operatorname{tr}\{$.$\} o operador traço, e U_{i}^{\prime}$ o transposto de $U_{i}$. Sabe-se que $\mathcal{M}^{n, s}$ com a norma acima é um espaço de Hilbert, com o produto interno dado, para $U, V \in \mathcal{M}^{n, s}$, por

$$
\langle U, V\rangle \triangleq \sum_{i \in \mathcal{O}} \operatorname{tr}\left\{U_{i}^{\prime} V_{i}\right\}
$$

Seja ainda $\Theta:=\{\theta(t) ; t=0,1, \ldots\}$ uma Cadeia de Markov a tempo discreto e nãohomogênea, tomando valores no conjunto $\mathcal{O}$ e tendo $\mathbb{P}(t) \in \mathbb{R}^{\eta \times \eta}, \forall t \geq 0$, como matriz de probabilidade de transição, sendo $\sum_{j \in \mathcal{O}}[\mathbb{P}(t)]_{i, j}=1, \forall i \in \mathcal{O}$ e $t \geq 0$. Denotamos $[G]_{i, j}$ para referirmo-nos ao elemento $(i, j)$ de uma dada matriz $G$, ou simplesmente $[G]_{i}$ caso $G$ seja um vetor. O vetor $\mu_{t} \in \mathbb{R}^{\eta}$ é definido como a distribuição de probabilidade da variável $\theta(t)$, assim temos que $\left[\mu_{t}\right]_{i}=\operatorname{Pr}(\theta(t)=i)$ e que, para $t \geq k$, a distribuição de $\theta(t)$ condicionada a $\mu_{k}$, denotada por $\mu_{t \mid k}$, pode ser obtida por $\mu_{t \mid k}=\left[\prod_{z=k}^{t-1} \mathbb{P}(z)\right]^{\prime} \mu_{k}$.

Considere também os operadores $\mathcal{E}^{t}(),. \mathcal{J}^{t}($.$) e \mathcal{V}^{t}\left(\right.$.) que levam elementos de $\mathcal{M}^{n, s}$ em $\mathcal{M}^{n, s}$, e $\mathcal{L}^{t}($.$) e \mathcal{T}^{t}\left(\right.$.) que mapeiam elementos de $\mathcal{M}^{n 0}$ em $\mathcal{M}^{n 0}$. Este operadores são utilizados de forma semelhante, como por exemplo, para $\mathcal{E}^{t}($.$) , temos \mathcal{E}^{t}(.) \triangleq$ $\left\{\mathcal{E}_{1}^{t}(),. \ldots, \mathcal{E}_{\eta}^{t}().\right\}$. Seja $A_{i}^{t} \triangleq A_{i}(t)+B_{i}(t) K_{i}^{t} \operatorname{com} A(t) \in \mathcal{M}^{n, n}, B(t) \in \mathcal{M}^{n, m} \mathrm{e}$ $K^{t} \in \mathcal{M}^{m, n}, \forall t \geq 0$, então, para $U \in \mathcal{M}^{n, s}, V \in \mathcal{M}^{n 0}$ e $i, j \in \mathcal{O}$, podemos definir os operadores acima como

$$
\begin{aligned}
& \mathcal{E}_{i}^{t}(U) \triangleq \sum_{j \in \mathcal{O}}[\mathbb{P}(t)]_{i j} U_{j}, \\
& \mathcal{J}_{i}^{t}(U) \triangleq\left(A_{i}^{t}\right)^{\prime} \mathcal{E}_{i}^{t}(U), \\
& \mathcal{V}_{i}^{t}(U) \triangleq \sum_{j \in \mathcal{O}}[\mathbb{P}(t)]_{j i} A_{j}^{t} U_{j}, \\
& \mathcal{L}_{i}^{t}(V) \triangleq\left(A_{i}^{t}\right)^{\prime} \mathcal{E}_{i}^{t}(V) A_{i}^{t} \quad \mathrm{e} \\
& \mathcal{T}_{i}^{t}(V) \triangleq \sum_{j \in \mathcal{O}}[\mathbb{P}(t)]_{j i} A_{j}^{t} V_{j}\left(A_{j}^{t}\right)^{\prime} .
\end{aligned}
$$

Cabe destacar que empregamos uma forma simplificada para representar a aplicação sucessiva destes operadores, por exemplo, considerando o operador $\mathcal{T}^{t}$, definimos $\mathcal{T}^{k, j} \triangleq$ 
$\mathcal{T}^{k}\left(\mathcal{T}^{k-1}\left(\mathcal{T}^{k-2}\left(\ldots \mathcal{T}^{j}(U) \ldots\right)\right)\right)$ quando $k \geq j$ e $\mathcal{T}^{k, j} \triangleq \mathcal{T}^{k}\left(\mathcal{T}^{k+1}\left(\mathcal{T}^{k+2}\left(\ldots \mathcal{T}^{j}(U) \ldots\right)\right)\right)$ para $k<j$.

Denotamos também os operadores lineares $\ddot{\phi}$ e $\hat{\phi}$ definidos abaixo. Para $U \in \mathcal{M}^{n, s}$, com $U_{i}=\left[\begin{array}{llll}u_{1}^{i} & u_{2}^{i} & \ldots & u_{s}^{i}\end{array}\right]$ sendo $u_{j}^{i} \in \mathbb{R}^{n, 1}$ a $j$-ésima coluna de $U_{i}$, então,

$$
\ddot{\phi}\left(U_{i}\right) \triangleq\left[\begin{array}{c}
u_{1}^{i} \\
u_{2}^{i} \\
\vdots \\
u_{s}^{i}
\end{array}\right] \in \mathbb{R}^{n \times s, 1} \quad \text { e } \hat{\phi}(U) \triangleq\left[\begin{array}{c}
\ddot{\phi}\left(U_{1}\right) \\
\ddot{\phi}\left(U_{2}\right) \\
\vdots \\
\ddot{\phi}\left(U_{\eta}\right)
\end{array}\right] \in \mathbb{R}^{n \times s \times \eta, 1}
$$

Além disto, utilizamos a função indicadora $\mathbb{1}_{\mathcal{A}}$, dada por

$$
\mathbb{1}_{\mathcal{A}}= \begin{cases}1, & \text { se a expressão } \mathcal{A} \text { é verdadeira } \\ 0, & \text { caso contrário, }\end{cases}
$$

para definir o que denominamos, respectivamente, variáveis de primeiro e segundo momento condicionado, como segue,

$$
\begin{aligned}
(i) \quad \chi_{i}(t) & \triangleq E\left\{x(t) \mathbb{1}_{\theta(t)=i}\right\} \\
(i i) \quad \mathcal{X}_{i}(t) & \triangleq E\left\{x(t) x(t)^{\prime} \mathbb{1}_{\theta(t)=i}\right\}
\end{aligned}
$$

Finalmente, consideramos o elemento $u_{k}^{T-1} \triangleq\{u(k), \ldots, u(T-1)\}$, para $0 \leq k \leq$ $T-1$, definido como uma sequência de leis controles $u(t) \in \mathcal{U}_{t}, k \leq t \leq T-1$, em que $\mathcal{U}_{t}$ é o conjunto de todas as funções vetoriais na forma $u(\theta(0), \ldots, \theta(t), x(0) \ldots, x(t), t)$.

\subsection{Definição do Problema}

Para compreender o problema tratado, vamos apresentar primeiramente o RLQ para SLSM com CHF e a solução disponível obtida de [14]. Para isto, considere o sistema a tempo discreto $\Psi$, definido em um espaço fundamental de probabilidade $\left(\Omega, \mathcal{F},\left\{\mathcal{F}_{t}\right\}, \mathcal{P}\right)$, dado por

$$
\Psi:\left\{\begin{array}{l}
x(t+1)=A_{\theta(t)}(t) x(t)+B_{\theta(t)}(t) u(t)+e_{\theta(t)}(t)+H_{\theta(t)}(t) \nu(t), t \geq 0 \\
x(0)=x_{0} \in \mathbb{R}^{n} \text { e } \theta(0)=\theta_{0} \in \mathcal{O}
\end{array}\right.
$$

em que o par $\left\{x(t) \in \mathbb{R}^{n} ; \theta(t) \in \mathcal{O}\right\}$ define o estado do sistema, $u(t) \in \mathbb{R}^{m}$ é o vetor de controle, $e_{i}(t) \in \mathbb{R}^{n}$ representa uma entrada exógena previamente conhecida e $\nu(t) \in \mathbb{R}^{l}$ 
é uma sequência de ruido independente e identicamente distribuído (i.i.d.) com média nula e, sem perda de generalidade, matriz de covariância dada por $E\left\{\nu(t) \nu(t)^{\prime}\right\}=\mathbb{I}_{l}$, em que $\mathbb{I}_{l}$ representa uma matriz identidade de ordem $l$. Considere também que o processo $\nu(t)$ seja independente de $x(0)$ de modo que $E(\nu(0) x(0))=0$. Seja ainda, a matriz $A_{i}(t)$, e similarmente para $B_{i}(t)$ e $H_{i}(t)$, conhecida, com dimensões apropriadas e uniformemente limitada, no sentido que $\exists \tilde{A} \in \mathbb{R}^{+}$tal que $\|A(t)\| \leq \tilde{A}$ para todo $t \geq 0$. Finalmente, considere a variável $\theta(t)$ como o estado de uma cadeia de Markov conhecida, de dimensões finitas e representada por $\mathbb{P}(t)$.

Além disto, considere também dois funcionais de custo, $p(t)$ e $q(t)$, definidos por

$$
\begin{aligned}
p(t)=( & \left.x(t)-\bar{x}_{\theta(t)}(t)\right)^{\prime} Q_{\theta(t)}(t)\left(x(t)-\bar{x}_{\theta(t)}(t)\right) \\
& +\left(u(t)-\bar{u}_{\theta(t)}(t)\right)^{\prime} R_{\theta(t)}(t)\left(u(t)-\bar{u}_{\theta(t)}(t)\right)
\end{aligned}
$$

e

$$
q(t)=\left(x(t)-\bar{x}_{\theta(t)}(t)\right)^{\prime} F_{\theta(t)}(t)\left(x(t)-\bar{x}_{\theta(t)}(t)\right)
$$

sendo $\bar{x}_{i}(t) \in \mathbb{R}^{n}$ e $\bar{u}_{i}(t) \in \mathbb{R}^{m}$, respectivamente, os alvos do estado e controle, conhecidos para todo $t \geq 0$ e $i \in \mathcal{O}$ e uniformemente limitados. Similarmente, sejam as matrizes $Q_{i}(t) \geq 0, R_{i}(t)>0$ e $F_{i}(t) \geq 0$ com dimensões apropriadas, conhecidas e uniformemente limitadas para todo $t \geq 0$ e $i \in \mathcal{O}$. Sejam ainda os seguintes funcionais de custo, conhecidos por custos de continuação,

$$
\mathcal{W}_{k}^{T}\left(\theta(k), x(k), u_{k}^{T-1}\right) \triangleq E\left\{\sum_{t=k}^{T-1} p(t)+q(T) \mid \mathfrak{G}_{k}\right\},
$$

para $k \in\{0, \ldots, T\}$, sendo $\mathfrak{G}_{k}$ o conjunto $\{x(t), \theta(t) ; t=0, \ldots, k\}$.

A função que fornece o custo de continuação ótimo (2.7), sujeito à $\Psi$, é denominada função valor e pode ser expressa por

$$
\overline{\mathcal{W}}_{k}^{T}(\theta(k), x(k)) \triangleq \inf _{u_{k}^{T-1}}\left[\mathcal{W}_{k}^{T}\left(\theta(k), x(k), u_{k}^{T-1}\right)\right]
$$

Assim, definimos como solução do problema intermediário, ou sequência de controle ótima, o conjunto de leis de controle $u_{k}^{* T-1}$, para o qual é válido

$$
\overline{\mathcal{W}}_{k}^{T}(\theta(k), x(k))=\mathcal{W}_{k}^{T}\left(\theta(k), x(k), u_{k}^{* T-1}\right) .
$$

O teorema seguinte apresenta as funções que definem $u_{k}^{* T-1} \mathrm{e} \overline{\mathcal{W}}_{k}^{T}(\theta(k), x(k))$ para o sistema $\Psi$. A prova pode ser obtida considerando o mesmo desenvolvimento apresen- 
tado em [14], contudo, para efeito didático, optamos por incluir no Apêndice A.2 uma versão mais estendida, no formato comum de programação dinâmica e com parâmetros variantes no tempo.

Teorema 2.2.1. A solução ótima para o problema intermediário associado ao sistema $\Psi$ pode ser dada por $u_{k}^{* T-1}=\left(u^{*}(k), \ldots, u^{*}(T-1)\right)$, em que $u^{*}(t)=r_{i}^{t}+K_{i}^{t} x$, para $0 \leq k \leq t \leq T-1$, sempre que $i=\theta(t)$ e $x=x(t)$, cujos parâmetros $r_{i}^{t}$ e $K_{i}^{t}$ são dados por

$$
\begin{gathered}
r_{i}^{t}=-\left(\Lambda_{i}^{t+1}\right)^{-1}\left[B_{i}(t)^{\prime}\left(\mathcal{E}_{i}^{t}\left(V^{t+1}\right) e_{i}(t)+\frac{1}{2} \mathcal{E}_{i}^{t}\left(v^{t+1}\right)\right)-R_{i}(t) \bar{u}_{i}(t)\right] e \\
K_{i}^{t}=-\left(\Lambda_{i}^{t+1}\right)^{-1} B_{i}(t)^{\prime} \mathcal{E}_{i}^{t}\left(V^{t+1}\right) A_{i}(t),
\end{gathered}
$$

com

$$
\Lambda_{i}^{t+1}=R_{i}(t)+B_{i}(t)^{\prime} \mathcal{E}_{i}^{t}\left(V^{t+1}\right) B_{i}(t)
$$

O custo de continuação incorrido por esta sequência de controle pode ser expresso pela função valor que segue,

$$
\overline{\mathcal{W}}_{k}^{T}(i, x)=x^{\prime} V_{i}^{k} x+x^{\prime} v_{i}^{k}+w_{i}^{k}
$$

com funções $V_{i}^{t}, v_{i}^{t}$ e $w_{i}^{t}$ atendendo à seguinte formação

$$
\begin{aligned}
V_{i}^{t}=Q_{i}(t)+ & \left(K_{i}^{t}\right)^{\prime} R_{i}(t) K_{i}^{t}+\left(A_{i}(t)+B_{i}(t) K_{i}^{t}\right)^{\prime} \mathcal{E}_{i}^{t}\left(V^{t+1}\right)\left(A_{i}(t)+B_{i}(t) K_{i}^{t}\right), \\
v_{i}^{t}= & \left(A_{i}(t)+B_{i}(t) K_{i}^{t}\right)^{\prime} \mathcal{E}_{i}^{t}\left(v^{t}\right)+2\left(A_{i}(t)+B_{i}(t) K_{i}^{t}\right)^{\prime} \mathcal{E}_{i}^{t}\left(V^{t}\right) e_{i}(t) \\
& -2\left(K_{i}^{t}\right)^{\prime} R(t) \bar{u}_{i}(t)-2 Q_{i}(t) \bar{x}_{i}(t), \quad e \\
w_{i}^{t}= & \mathcal{E}_{i}^{t}\left(w^{t+1}\right)+\bar{x}_{i}(t)^{\prime} Q_{i}(t) \bar{x}_{i}(t)+\bar{u}_{i}^{\prime}(t) R_{i}(t) \bar{u}_{i}(t)+e_{i}^{\prime}(t) \mathcal{E}_{i}^{t}\left(V^{t+1}\right) e_{i}(t) \\
& +\mathcal{E}_{i}^{t}\left(v^{t+1}\right)^{\prime} e_{i}(t)-\left(r_{i}^{t}\right)^{\prime} \Lambda_{i}^{t+1} r_{i}^{t}+\operatorname{tr}\left[H_{i}(t) H_{i}(t)^{\prime} \mathcal{E}_{i}^{t}\left(V^{t+1}\right)\right] \\
\operatorname{com} V_{i}^{T}= & F_{i}(T), v_{i}^{T}=-2 F_{i}(T) \bar{x}_{i}(T) e w_{i}^{T}=\bar{x}_{i}^{\prime}(T) F_{i}(T) \bar{x}_{i}(T) .
\end{aligned}
$$

Demonstração: Vide Seção A.1 do Apêndice.

Para facilitar a exposição das demais hipóteses e a compreensão da definição de estabilidade empregada, consideramos uma versão particularizada de $\Psi$, em que $u(t)=$ $K_{\theta(t)}^{t} x(t)$, para algum $K^{t} \in \mathcal{M}^{m, n}, e_{i}(t)=0$ e $H_{i}(t)=0$, para todo $t \geq 0$ e $i \in \mathcal{O}$, 
definido como

$$
\breve{\Psi}:\left\{\begin{array}{l}
x(t+1)=\left(A_{\theta(t)}(t)+B_{\theta(t)}(t) K_{\theta(t)}^{t}\right) x(t), t \geq 0 \\
x(0)=x_{0} \in \mathbb{R}^{n} \quad \text { e } \quad \theta(0)=\theta_{0} \in \mathcal{O}
\end{array}\right.
$$

Assim, associados ao sistema $\breve{\Psi}$, definimos o primeiro e segundo momento representados por $\breve{\chi}$ e $\breve{\mathcal{X}}$, e consideramos também um custo referente ao regulador linear quadrático, obtido de (2.7) considerando-se nulos os alvos de estado e controle, dado por

$$
\begin{gathered}
\breve{\mathcal{W}}_{k}^{T}(i, x)=E\left(\sum_{t=k}^{T-1} x(t)^{\prime}\left(Q_{\theta(t)}(t)+\left(K_{\theta(t)}^{t}\right)^{\prime} R_{\theta(t)} K_{\theta(t)}^{t}\right) x(t)\right. \\
\left.+x(T)^{\prime} F_{\theta(T)}(T) x(T) \mid \mathfrak{G}_{k}\right)
\end{gathered}
$$

para $\theta(k)=i \in \mathcal{O}$ e $x(k)=x \in \mathbb{R}^{n}$.

A proposição abaixo foi extraida de [14] e mostra um resultado bastante conhecido na literatura, sendo importante para reapresentarmos o custo (2.17) em uma forma mais conveniente à definição de detetabilidade utilizada neste trabalho.

Proposição 2.2.2. Seja uma sequência $L^{t} \in \mathcal{M}^{n 0}$, definida para $0 \leq t \leq T$ e dada por $L^{t}=Q^{t}+\mathcal{L}^{t}\left(L^{t+1}\right)$, com $Q^{t} \triangleq Q(t)+\left(K^{t}\right)^{\prime} R(t) K^{t}$ e $L^{T}=Q^{T}=F(T)$. Então, podemos reapresentar o custo (2.17) como $\breve{\mathcal{W}}_{k}^{T}(i, x)=\sum_{t=k}^{T}\left\langle Q^{t}, \breve{\mathcal{X}}^{t}\right\rangle=\left\langle L^{k}, \breve{\mathcal{X}}^{k}\right\rangle$.

Demonstração: Vide Seção A.2 do Apêndice.

Observação 2.2.3. Tendo em vista a Proposição 2.2.2, vamos estender a definição do custo (2.17) para $\breve{\mathcal{W}}_{k}^{T}(\mathcal{X})=\sum_{t=k}^{T}\left\langle Q^{t}, \breve{\mathcal{X}}^{t}\right\rangle \operatorname{com} \breve{\mathcal{X}}^{k}=\mathcal{X} \in \mathcal{M}^{n 0}$.

Na sequência, apresentamos as hipóteses de detetabilidade uniforme [1, 9], de custo uniformemente limitado e o conceito de sistema exponencialmente estável, fundamentais para as demonstrações pretendidas.

Definição 2.2.4. Definimos $(\Psi, \mathcal{W})$ como uniformemente detetável se existem inteiros $T_{d}, t_{d} \geq 0$ e escalares $0 \leq \delta<1$ e $\gamma>0$ tais que $\breve{\mathcal{W}}_{k}^{k+T_{d}}(\mathcal{X}) \geq \gamma\|\mathcal{X}\|$ sempre que $\left\|\breve{\mathcal{X}}^{k+t_{d}}\right\| \geq \delta\|\mathcal{X}\|$, para qualquer $\breve{\mathcal{X}}^{k}=\mathcal{X} \in \mathcal{M}^{n 0}$ e $k \geq 0$.

Definição 2.2.5. O custo $\breve{\mathcal{W}}$ é dito uniformemente limitado se existe um $\breve{W}^{+} \in \mathbb{R}^{+}$ para o qual $\breve{\mathcal{W}}_{k}^{T}(\mathcal{X})<\breve{\mathcal{W}}^{+}\|\mathcal{X}\|$, para todo $\breve{\mathcal{X}}^{k}=\mathcal{X} \in \mathcal{M}^{n 0}, k \geq 0$ e $T \geq k$. 
Definição 2.2.6. Dizemos que uma sequência qualquer $z^{t} \in \mathbb{R}^{+}$converge exponencialmente se existem reais $\lambda<1$ e $\phi$ tais que $z^{t} \leq \phi \lambda^{t} z^{0}$, para todo $t \geq 0$.

Definição 2.2.7. Dizemos que a sequência $K^{t} \in \mathcal{M}^{m, n}$, para $t \geq 0$, estabiliza exponencialmente o sistema $\Psi$, se a sequência $\left\|\breve{\mathcal{X}}^{t}\right\|$ converge exponencialmente para todo $\breve{\mathcal{X}}^{0} \in \mathcal{M}^{n 0}$.

Assim, considerando que $(\Psi, \mathcal{W})$ atende ao conceito de detetabilidade uniforme e que o custo $\breve{\mathcal{W}}$ seja uniformemente limitado, nosso objetivo é mostrar que o CMLP, dado por

$$
\begin{gathered}
\overline{\mathcal{W}}(\theta(0), x(0))=\inf _{u_{0}}\left[\limsup _{T \rightarrow \infty}\left\{\frac{1}{T+1} \mathcal{W}_{0}^{T}\left(\theta(0), x(0), u_{0}^{T-1}\right)\right\}\right] \\
\text { s.a. }(2.6) .
\end{gathered}
$$

está bem definido, ou seja, queremos mostrar que existe $\overline{\mathcal{W}}(\theta(0), x(0))$, para todo $x(0)$ e $\theta(0)$. Para isto, demonstramos primeiramente na Seção 2.3 que, nas condições propostas, a sequência $K^{t} \in \mathcal{M}^{m, n}$ estabiliza exponencialmente o sistema $\Psi$.

\subsection{Estabilidade Exponencial do Regulador Linear Qua- drático}

O Teorema 2.3.3 abaixo utiliza a definição de detetabilidade uniforme e o limitante uniforme para $\breve{\mathcal{W}}_{k}^{T}(\mathcal{X})$ para concluir sobre a convergência exponencial da série $\left\|\breve{\mathcal{X}}^{t}\right\|$. $\mathrm{Na}$ literatura, podemos encontrar resultados semelhantes para SLSM invariantes no tempo, vinculados à MS-estabilidade [7]. Entretanto, estes resultados não são suficientes para a demonstração do Lema 2.4.4, apresentado na Seção 2.4. Assim, obtivemos a prova adaptando diretamente as idéias de [9, Teorema 1], em que se apresenta a convergência exponencial do estado $x(t)$ para sistemas não-lineares e sem saltos. Para isto, iniciamos apresentando a Proposição 2.3.1, necessária para a demonstração do Teorema 2.3.3, e, principalmente, para a obtenção dos demais resultados dispostos na Seção 2.4. Esta proposição foi adaptada de [10] e relaciona a evolução das sequências $\breve{\chi}_{i}^{t}$ e $\breve{\mathcal{X}}_{i}^{t}$ aos operadores $\mathcal{V}^{t}($.$) e \mathcal{T}^{t}($.$) . A demonstração será apresentada pois, neste caso,$ trata-se de um sistema variante no tempo e, além disto, decidimos por uma versão um pouco mais detalhada da prova.

Proposição 2.3.1. As sequências $\breve{\chi}^{t}$ e $\breve{\mathcal{X}}^{t}$, podem ser calculados de forma recursiva por (i) $\breve{\chi}^{t+1}=\mathcal{V}^{t}\left(\breve{\chi}^{t}\right) e\left(\right.$ ii) $\breve{\mathcal{X}}^{t+1}=\mathcal{T}^{t}\left(\breve{\mathcal{X}}^{t}\right)$. 
Demonstração: (i) Por definição temos que

$$
\breve{\chi}_{i}^{t+1}=E\left\{x(t+1) \mathbb{1}_{\theta(t+1)=i}\right\} .
$$

Logo, podemos escrever

$$
\begin{aligned}
& =\sum_{j \in \mathcal{O}} E\left\{\left(A_{j}(t)+B_{j}(t) K_{j}^{t}\right) x(t) \mathbb{1}_{\theta(t+1)=i} \mathbb{1}_{\theta(t)=j}\right\} \\
& =\sum_{j \in \mathcal{O}} A_{j}^{t} E\left\{x(t) \mathbb{1}_{\theta(t)=j} \mathbb{1}_{\theta(t+1)=i}\right\} \\
& =\sum_{j \in \mathcal{O}} A_{j}^{t} E\{x(t) \mid \theta(t)=j, \theta(t+1)=i\} \mathcal{P}(\theta(t)=j, \theta(t+1)=i) \\
& \left.=\sum_{j \in \mathcal{O}} A_{j}^{t} E\{x(t) \mathcal{P}(\theta(t)=j)\} \mathcal{P}(\theta(t+1)=i) \mid \theta(t)=j\right) \\
& =\sum_{j \in \mathcal{O}}[\mathbb{P}(t)]_{j i} A_{j}^{t} E\left\{x(t) \mathbb{1}_{\theta(t)=j}\right\} \\
& =\sum_{j \in \mathcal{O}}[\mathbb{P}(t)]_{j i} A_{j}^{t} \breve{\chi}_{j}^{t} \\
& =\mathcal{V}_{i}^{t}\left(\breve{\chi}^{t}\right) .
\end{aligned}
$$

Portanto, $\breve{\chi}^{t+1}=\mathcal{V}^{t}\left(\breve{\chi}^{t}\right)$.

(ii) A prova pode ser obtida de maneira análoga à apresentada em (i). Para o caso invariante no tempo, consulte também [10], Proposição 3.1.

O lema seguinte é uma adaptação direta de [9, Lema 1]. A prova envolve o fato que a sequência $\breve{\mathcal{X}}^{t}$ visita uma bola de raio arbitrario, dentro de um intervalo de tempo $[0, T]$ dependente de $r$ e $\breve{\mathcal{W}}^{+}[8]$.

Lema 2.3.2. Seja o sistema $(\Psi, \mathcal{W})$ uniformemente detetável e $\breve{\mathcal{W}}$ uniformemente limitado. Considere também que exista um real $\iota>0$ de modo que $\left\|\breve{\mathcal{X}}^{k+1}\right\| \leq \iota\left\|\breve{\mathcal{X}}^{k}\right\|$ para qualquer $\breve{\mathcal{X}}^{k} \in \mathcal{M}^{n 0}$ e $k \geq 0$. Então, para $r>0$ e $T_{1}$ satisfazendo

$$
T_{1} \geq T_{0}\left(\breve{\mathcal{W}}^{+}, r\right)=\bar{T}\left(\frac{\breve{\mathcal{W}}^{+}}{\gamma^{r}}\left(1-\bar{T} \log _{\delta}(\iota)\right)+\log _{\delta}(r)+1\right)
$$

em que $\bar{T}=\max \left\{T_{d}, t_{d}\right\}$, temos que a desigualdade

$$
0<\breve{\mathcal{W}}_{k}^{T_{2}+k}(\breve{\mathcal{X}})-\breve{\mathcal{W}}_{k}^{T_{1}+k}(\breve{\mathcal{X}}) \leq r \breve{\mathcal{W}}^{+}\|\breve{\mathcal{X}}\|
$$


é válida para qualquer $T_{2} \geq T_{1}$ e $\forall \breve{\mathcal{X}} \in \mathcal{M}^{n 0}$ e $k \geq 0$.

Teorema 2.3.3. Seja o sistema $(\Psi, \mathcal{W})$ uniformemente detetável e $\breve{\mathcal{W}}$ uniformemente limitado. Então, a sequência $\left\|\breve{\mathcal{X}}^{t}\right\|$ é exponencialmente convergente $\forall \breve{\mathcal{X}}^{0} \in \mathcal{M}^{n 0}$.

Demonstração: A prova que segue é uma adaptação de [9, Teorema 1]. Em suma, mostramos que a função de Lyapunov $J_{k}^{T}: \mathcal{M}^{n 0} \rightarrow \mathbb{R}^{+}$, definida para $k \geq 0$ e dada por

$$
J_{k}^{T}(\mathcal{X}) \triangleq \sigma\|\mathcal{X}\|+\breve{\mathcal{W}}_{k}^{k+T}(\mathcal{X})
$$

sempre que $\breve{\mathcal{X}}^{k}=\mathcal{X} \in \mathcal{M}^{n 0}$, atende à desigualdade abaixo,

$$
J_{k_{n+1}}^{T}\left(\breve{\mathcal{X}}^{k_{n+1}}\right)<\alpha J_{k_{n}}^{T}\left(\breve{\mathcal{X}}^{k_{n}}\right)
$$

em que $0<\alpha<1$, para $T \geq T_{0}\left(\breve{\mathcal{W}}^{+}, r\right)$ e sequência de tempo $k_{n}$ crescente dada por $k_{0}=0$ e $k_{n+1}=k_{n}+t_{d}$ ou $k_{n+1}=k_{n}+\bar{T}+1$. A partir deste resultado, mostramos $\left\|\mathcal{X}^{t}\right\|$ é exponencialmente convergente. Primeiramente, note, com o auxílio da Proposição 2.3.1, que existe um real $\iota>0$ para o qual $\left\|\breve{\mathcal{X}}^{t+1}\right\| \leq \iota\left\|\breve{\mathcal{X}}^{t}\right\|$ para qualquer $\breve{\mathcal{X}}^{t} \in \mathcal{M}^{n 0} \mathrm{e}$ $t \geq 0$. Esta desigualdade, que denominamos condição de crescimento, é válida pois as coleções de matrizes $A(t)$ e $B(t)$ são uniformemente limitadas e é possível verificar, a partir de $R(t)>0$ e da existência de um limitante para $\left\|V^{0}\right\|$ (vide prova do Teorema 2.4.6), que a sequência $K^{t}$ também é limitada. Por outro lado, de [9, Observação 3], sabemos que existe um escalar $r>0$, cujo papel nesta prova será elucidado adiante, que atende a,

$$
r<\frac{\gamma}{\breve{\mathcal{W}}^{+}}\left[\left(\sum_{l=1}^{\bar{T}} \iota^{l}\right)+\left(\frac{1-\iota^{\bar{T}}}{\delta-1}\right)\left(\sum_{l=1}^{t_{d}} \iota^{l}\right)\right]^{-1} .
$$

A partir da equação (2.19) e da Proposição 2.2.2, podemos escrever

$$
\begin{aligned}
J_{k}^{T}\left(\breve{\mathcal{X}}^{k}\right)-J_{k+1}^{T}\left(\breve{\mathcal{X}}^{k+1}\right)=\sigma \| & \breve{\mathcal{X}}^{k}\|-\sigma\| \breve{\mathcal{X}}^{k+1} \|+\left\langle Q^{k}, \breve{\mathcal{X}}^{k}\right\rangle \\
& +\breve{\mathcal{W}}_{k+1}^{T+k}\left(\breve{\mathcal{X}}^{k+1}\right)-\breve{\mathcal{W}}_{k+1}^{T+k+1}\left(\breve{\mathcal{X}}^{k+1}\right)
\end{aligned}
$$

Mas observando o Lema 2.3.2 sabemos que é válida a seguinte relação,

$$
\breve{\mathcal{W}}_{k+1}^{T+k+1}\left(\breve{\mathcal{X}}^{k+1}\right)-\breve{\mathcal{W}}_{k+1}^{T+k}\left(\breve{\mathcal{X}}^{k+1}\right) \leq r \breve{\mathcal{W}}^{+}\left\|\breve{\mathcal{X}}^{k+1}\right\| \leq r \breve{\mathcal{W}}^{+} \iota\left\|\breve{\mathcal{X}}^{k}\right\|
$$

Assim, avaliamos dois casos distintos que seguem. Caso a) $\left\|\breve{\mathcal{X}}^{k+t d}\right\| \geq \delta\left\|\breve{\mathcal{X}}^{k}\right\|$. Substituindo (2.23) em (2.22) é possível obter, para qualquer $k \leq l \leq k+\bar{T}$, as desigualdades

$$
J_{l}^{T}\left(\breve{\mathcal{X}}^{l}\right)-J_{l+1}^{T}\left(\breve{\mathcal{X}}^{l+1}\right)>\sigma\left\|\breve{\mathcal{X}}^{l}\right\|-\sigma\left\|\breve{\mathcal{X}}^{l+1}\right\|+\left\langle Q^{l}, \breve{\mathcal{X}}^{l}\right\rangle-r \breve{\mathcal{W}}^{+} \iota\left\|\breve{\mathcal{X}}^{l}\right\|
$$


Somando em $l$ as desigualdades acima e utilizando a condição de crescimento é possível escrever,

$$
J_{k}^{T}\left(\breve{\mathcal{X}}^{k}\right)-J_{k+\bar{T}+1}^{T}\left(\breve{\mathcal{X}}^{k+\bar{T}+1}\right)>\sigma\left\|\breve{\mathcal{X}}^{k}\right\|-\sigma\left\|\breve{\mathcal{X}}^{k+\bar{T}+1}\right\|+\sum_{l=k}^{k+\bar{T}}\left\langle Q^{l}, \breve{\mathcal{X}}^{l}\right\rangle-r \breve{\mathcal{W}}^{+}\left[\sum_{l=1}^{\bar{T}+1} \iota^{l}\right]\left\|\breve{\mathcal{X}}^{k}\right\| .
$$

Considerando a definição de $\iota$ podemos verificar que $\sigma\left\|\breve{\mathcal{X}}^{k}\right\|-\sigma\left\|\breve{\mathcal{X}}^{k+\bar{T}+1}\right\| \geq \sigma(1-$ $\left.\iota^{\bar{T}+1}\right)\left\|\breve{\mathcal{X}}^{k}\right\|$. Pela definição de detetabilidade, temos que o terceiro termo de (2.25) é limitado por baixo por $\gamma\left\|\breve{\mathcal{X}}^{k}\right\|$, uma vez que $\bar{T} \geq T_{d}$. Logo, a desigualdade acima pode ser reescrita como

$$
J_{k}^{T}\left(\breve{\mathcal{X}}^{k}\right)-J_{k+\bar{T}+1}^{T}\left(\breve{\mathcal{X}}^{k+\bar{T}+1}\right)>\zeta\left\|\breve{\mathcal{X}}^{k}\right\|
$$

na qual,

$$
\zeta \triangleq\left[\sigma\left(1-\iota^{\bar{T}+1}\right)+\gamma-r \breve{\mathcal{W}}^{+} \sum_{l=1}^{\bar{T}+1} \iota^{l}\right]
$$

Observe que podemos definir,

$$
\bar{\sigma} \triangleq\left(r \breve{\mathcal{W}}^{+} \sum_{l=1}^{\bar{T}+1} \iota^{l}-\gamma\right) /\left(1-\iota^{\bar{T}+1}\right)
$$

e então obter, $\zeta=(\sigma-\bar{\sigma})\left(1-\iota^{\bar{T}+1}\right)$. Escolhendo $\sigma<\bar{\sigma}$ e um $\iota>1$ arbitrário, obtemos $\zeta>0$. Note que $\left(\sigma+\breve{\mathcal{W}}^{+}\right)\left\|\breve{\mathcal{X}}^{k}\right\| \geq \sigma\left\|\breve{\mathcal{X}}^{k}\right\|+\breve{\mathcal{W}}_{k}^{T+k}\left(\breve{\mathcal{X}}^{k}\right)=J_{k}^{T}\left(\breve{\mathcal{X}}^{k}\right)$. Substituindo esta ultima expressão em (2.26) é possível obter

$$
\alpha_{1} J_{k}^{T}\left(\breve{\mathcal{X}}^{k}\right)>J_{k+\bar{T}+1}^{T}\left(\breve{\mathcal{X}}^{k+\bar{T}+1}\right)
$$

em que $\alpha_{1}=1-\zeta /\left(\sigma+\breve{\mathcal{W}}^{+}\right)$. Utilizando o fato $\gamma\left\|\breve{\mathcal{X}}^{k}\right\| \leq \breve{\mathcal{W}}_{k}^{T+k}\left(\breve{\mathcal{X}}^{k}\right) \leq \breve{\mathcal{W}}^{+}\left\|\breve{\mathcal{X}}^{k}\right\|$ obtemos a desigualdade $\zeta<\sigma+\gamma \leq \sigma+\breve{\mathcal{W}}^{+}$para qualquer $T \geq T_{d}$. A partir desta última e lembrando que $\zeta>0$, é fácil observar que $0<\alpha_{1}<1$. Caso b) $\left\|\breve{\mathcal{X}}^{k+t_{d}}\right\|<$ $\delta\left\|\breve{\mathcal{X}}^{k}\right\|$. De modo similar ao caso anterior, substituindo (2.23) em (2.22) obtemos, para qualquer $k \leq l \leq k+t_{d}-1$, desigualdades idênticas à (2.24) e, consequentemente, o seguinte,

$$
J_{k}^{T}\left(\breve{\mathcal{X}}^{k}\right)-J_{k+t_{d}}^{T}\left(\breve{\mathcal{X}}^{k+t_{d}}\right)>\sigma\left\|\breve{\mathcal{X}}^{k}\right\|-\sigma\left\|\breve{\mathcal{X}}^{k+t_{d}}\right\|+\sum_{l=k}^{k+t_{d}-1}\left\langle Q^{l}, \breve{\mathcal{X}}^{l}\right\rangle-r \breve{\mathcal{W}}^{+}\left[\sum_{l=1}^{t_{d}} \iota^{l}\right]\left\|\breve{\mathcal{X}}^{k}\right\| .
$$


Note que $\sigma\left\|\breve{\mathcal{X}}^{k}||-\sigma\right\| \mathcal{X}^{k+t_{d}}\|\geq \sigma(1-\delta)\| \breve{\mathcal{X}}^{k} \|$ e então podemos escrever

$$
J_{k}^{T}\left(\breve{\mathcal{X}}^{k}\right)-J_{k+t_{d}}^{T}\left(\breve{\mathcal{X}}^{k+t_{d}}\right)>\zeta^{\prime}\left\|\breve{\mathcal{X}}^{k}\right\|
$$

em que

$$
\zeta^{\prime} \triangleq \sigma(1-\delta)-r \breve{\mathcal{W}}^{+}\left[\sum_{l=1}^{t_{d}} \iota^{l}\right] .
$$

Lembrando que $0 \leq \delta<1$, e tomando $\sigma>\underline{\sigma}$, em que $\underline{\sigma} \triangleq r \breve{\mathcal{W}}^{+}\left[\sum_{l=1}^{t_{d}} \iota^{l}\right] /(1-\delta)$, obtemos $\zeta^{\prime}>0$. Novamente, utilizando $\left(\sigma+\breve{\mathcal{W}}^{+}\right)\left\|\breve{\mathcal{X}}^{k}\right\| \geq \sigma\left\|\breve{\mathcal{X}}^{k}\right\|+\breve{\mathcal{W}}_{k}^{k+T}\left(\breve{\mathcal{X}}^{k}\right)=$ $J_{k}^{T}\left(\breve{\mathcal{X}}^{k}\right)$, obtemos

$$
\alpha_{2} J_{k}^{T}\left(\breve{\mathcal{X}}^{k}\right)>J_{k+t_{d}}^{T}\left(\breve{\mathcal{X}}^{k+t_{d}}\right)
$$

na qual $\alpha_{2}=1-\zeta^{\prime} /\left(\sigma+\breve{\mathcal{W}}^{+}\right)$. Utilizando os mesmos argumentos como em (2.27) é possível mostrar que $0<\alpha_{2}<1$. Note que a existência de $\sigma$ no intervalo aberto $\underline{\sigma}<\sigma<\bar{\sigma}$ é garantida por $r>0$ atendendo a (2.21). Finalmente, definindo $\alpha=\max \left\{\alpha_{1}, \alpha_{2}\right\}$, e escolhendo $k_{n+1}=k_{n}+\bar{T}+1$ se $\left\|\breve{\mathcal{X}}^{k+t_{d}}\right\| \geq \delta\left\|\breve{\mathcal{X}}^{k}\right\|$ ou $k_{n+1}=k_{n}+t_{d}$ se $\left\|\breve{\mathcal{X}}^{k+t_{d}}\right\|<\delta\left\|\breve{\mathcal{X}}^{k}\right\|$, obtemos (2.20). Para encontrarmos uma envoltória exponencialmente convergente para $\left\|\breve{\mathcal{X}}^{t}\right\|$, escrevemos, a partir de $(2.20), J_{k_{n}}^{T}\left(\breve{\mathcal{X}}^{k_{n}}\right)=\sigma\left\|\breve{\mathcal{X}}^{k_{n}}\right\|+\mathcal{W}_{k_{n}}^{T+k_{n}}\left(\breve{\mathcal{X}}^{k_{n}}\right)<\alpha^{n} J_{0}^{T}\left(\breve{\mathcal{X}}^{0}\right)$. Com isto, e lembrando que $J_{0}^{T}\left(\breve{\mathcal{X}}^{0}\right) \leq\left(\sigma+\breve{\mathcal{W}}^{+}\right)\left\|\breve{\mathcal{X}}^{0}\right\|$, obtemos

$$
\left\|\breve{\mathcal{X}}^{k_{n}}\right\|<\frac{1}{\sigma} \alpha^{n} J_{0}^{T}\left(\breve{\mathcal{X}}^{0}\right) \leq \alpha^{n} \frac{\left(\sigma+\breve{\mathcal{W}}^{+}\right)}{\sigma}\left\|\breve{\mathcal{X}}^{0}\right\|
$$

Utilizando a condição de crescimento limitado, temos que $\left\|\breve{\mathcal{X}}^{t}\right\| \leq \iota^{\bar{T}+1}|| \breve{\mathcal{X}}^{k_{n}} \|$ para $k_{n} \leq t<k_{n+1}$. Ainda, a partir da construção da sequência $k_{n}$, podemos inferir que $n \geq t /(\bar{T}+1)$ então,

$$
\left\|\breve{\mathcal{X}}^{t}\right\|<\iota^{\bar{T}+1} \alpha^{n} \frac{\left(\sigma+\breve{\mathcal{W}}^{+}\right)}{\sigma}\left\|\breve{\mathcal{X}}^{0}\right\|<\iota^{\bar{T}+1} \frac{\left(\sigma+\breve{\mathcal{W}}^{+}\right)}{\sigma}\left(\alpha^{\frac{1}{\bar{T}+1}}\right)^{t}\left\|\breve{\mathcal{X}}^{0}\right\|
$$

Observe que podemos definir $\phi=\iota^{\bar{T}+1}\left(\sigma+\breve{\mathcal{W}}^{+}\right) / \sigma$ e $\lambda=\left(\alpha^{\frac{1}{\bar{T}+1}}\right)<1$ e escrever $\left\|\breve{\mathcal{X}}^{t}\right\|<\phi \lambda^{t}\left\|\breve{\mathcal{X}}^{0}\right\|$. Portanto, observando a Definição 2.2.6, podemos concluir que a sequência $\left\|\breve{\mathcal{X}}^{t}\right\|$ é exponencialmente convergente para qualquer $\breve{\mathcal{X}}^{0} \in \mathcal{M}^{n 0}$.

Corolário 2.3.4. Seja o sistema $(\Psi, \mathcal{W})$ uniformemente detetável e $\breve{\mathcal{W}}$ uniformemente limitado. Então, a sequência $K^{t} \in \mathcal{M}^{m, n}$, para $t \geq 0$ estabiliza exponencialmente o sistema $\Psi, \forall \breve{\mathcal{X}}^{0} \in \mathcal{M}^{n 0}$. 
Demonstração: A prova é obtida diretamente combinando-se o resultado do Teorema 2.3.3 e da Definição 2.2.7.

\subsection{Existência do CMLP}

Para mostrar o principal resultado deste capítulo, apresentado pelo Teorema 2.4.6, utilizamos o Teorema 2.2.1 para reescrevermos (2.18) como o seguinte,

$$
\overline{\mathcal{W}}(\theta(0), x(0))=\lim \sup _{T \rightarrow \infty} \frac{1}{T+1}\left\{x(0)^{\prime} V_{\theta(0)}^{0} x(0)+x(0)^{\prime} v_{\theta(0)}^{0}+w_{\theta(0)}^{0}\right\}
$$

o que torna evidente a relação entre a existência de $\overline{\mathcal{W}}(\theta(0), x(0))$ e o comportamento das variáveis $V_{i}^{0}, v_{i}^{0}$ e $w_{i}^{0}$ quando $T \rightarrow \infty$. Neste sentido, apresentamos os Lemas 2.4.4 e 2.4.5 abaixo que, como veremos adiante, estão intimamente ligados à $v_{i}^{0}$ e $w_{i}^{0}$, respectivamente, para um horizonte de tempo infinito. Antes porém, apresentamos alguns resultados essenciais para a prova do Lema 2.4.4. O Lema 2.4.1 verifica a dualidade entre os operadores $\mathcal{V}^{t}($.$) e \mathcal{J}^{t}($.$) , a prova pode ser obtida utilizando passagens idênti-$ cas a [10], Proposição 3.2, porém, incluindo-se a dependência temporal dos operadores. Já o Lema 2.4.3, combina a Proposição 2.4.2, extraída de [10, Proposição 3.6] com o resultado do Teorema 2.3.3 para demonstrar a convergência exponencial de $\left\|\breve{\chi}^{t}\right\|$.

Lema 2.4.1. Os operadores $\mathcal{V}^{t}\left(\right.$.) e $\mathcal{J}^{t}($.$) são duais, no sentido de que é válida a$ seguinte propriedade,

$$
\left\langle V, \mathcal{V}^{t}(U)\right\rangle=\left\langle\mathcal{J}^{t}(V), U\right\rangle
$$

para quaisquer $U, V \in \mathcal{M}^{n, r}$.

Proposição 2.4.2. Sejam as variáveis $\chi(t)$ e $\mathcal{X}(t)$ definidas em (2.5). Então é válida a seguinte desigualdade

$$
\|\hat{\phi}(\chi(t))\|^{2} \leq n\|\mathcal{X}(t)\|
$$

Lema 2.4.3. Seja o sistema $(\Psi, \mathcal{W})$ uniformemente detetável e $\breve{\mathcal{W}}$ uniformemente limitado. Então, a sequência $\left\|\breve{\chi}^{t}\right\|$ é exponencialmente convergente $\forall \breve{\chi}^{0} \in \mathcal{M}^{n, 1}$.

Demonstração: A prova pode ser obtida combinando-se os resultados da Proposição 2.4.2 e do Lema 2.3.3. 


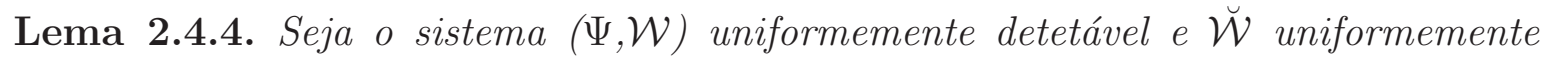
limitado. Seja ainda $l^{t} \in \mathcal{M}^{n, 1}$ definido para $0 \leq t \leq T$ e dado por $l^{t}=(A(t)+$ $\left.B(t) K^{t}\right)^{\prime} \mathcal{E}^{t}\left(l^{t+1}\right)+g(t)$, com $l^{T}=g(T)$, em que $g(t) \in \mathcal{M}^{n, 1}$ é uniformemente limitada, no sentido que $\exists m_{g} \in \mathbb{R}$ de tal modo que $\left\|g_{i}(t)\right\| \leq m_{g}, \forall t \geq 0$ e $i \in \mathcal{O}$. Então, é válido que

$$
\lim \sup _{T \rightarrow \infty}\left[\frac{\left\|l_{i}^{0}\right\|}{T+1}\right]=0, \forall i \in \mathcal{O}
$$

Demonstração: Iniciamos considerando um funcional de custo auxiliar, dado por,

$$
\mathcal{Z}^{T}=\sum_{t=0}^{T} E\left\{g_{\theta(t)}(t)^{\prime} x(t)\right\}
$$

em que $x(t)$ é o vetor de estados associado ao sistema $\breve{\Psi}$. Então,

$$
\begin{aligned}
\mathcal{Z}^{T} & =E\left\{\sum_{t=0}^{T} \sum_{i \in \mathcal{O}} \operatorname{tr}\left(g_{i}(t)^{\prime} x(t) \mathbb{1}_{\theta(t)=i}\right)\right\} \\
& =\sum_{t=0}^{T} \sum_{i \in \mathcal{O}} \operatorname{tr}\left(g_{i}(t)^{\prime} E\left\{x(t) \mathbb{1}_{\theta(t)=i}\right\}\right) \\
& =\sum_{t=0}^{T} \sum_{i \in \mathcal{O}} \operatorname{tr}\left(g_{i}(t)^{\prime} \breve{\chi}_{i}^{t}\right)=\sum_{t=0}^{T}\left\langle g(t), \breve{\chi}^{t}\right\rangle .
\end{aligned}
$$

Aplicando a desigualdade de Cauchy-Schwarz à equação anterior e utilizando o resultado do Lema 2.4.3, podemos escrever

$$
\mathcal{Z}^{T} \leq \sum_{t=0}^{T}\|g(t)\|\left\|\breve{\chi}^{t}\right\| \leq \sum_{t=0}^{T} \eta m_{g} \phi \lambda^{t}\left\|\breve{\chi}^{0}\right\| \leq \frac{1}{1-\lambda} \eta m_{g} \phi\left\|\breve{\chi}^{0}\right\|
$$

para algum escalar $\phi>0$ e $0 \leq \lambda<1$. Logo, definindo $\overline{\mathcal{Z}}\left(\breve{\chi}^{0}\right) \triangleq \frac{1}{1-\lambda} \eta m_{g} \phi\left\|\breve{\chi}^{0}\right\|$, temos que $\mathcal{Z}^{T} \leq \overline{\mathcal{Z}}\left(\breve{\chi}^{0}\right), \forall T \geq 0$. Por outro lado, com o auxílio da Proposição 2.3.1 e do Lema 2.4.1, podemos reescrever a equação (2.32) do seguinte modo,

$$
\mathcal{Z}^{T}=\sum_{t=0}^{T}\left\langle g(t), \mathcal{V}^{t-1,0}\left(\breve{\chi}^{0}\right)\right\rangle=\sum_{t=0}^{T}\left\langle\mathcal{J}^{0, t-1}(g(t)), \breve{\chi}^{0}\right\rangle=\left\langle\sum_{t=0}^{T} \mathcal{J}^{0, t-1}(g(t)), \breve{\chi}^{0}\right\rangle
$$

com a convenção $\mathcal{V}^{-1,0}(U)=\mathcal{J}^{0,-1}(U)=U$, para qualquer $U \in \mathcal{M}^{n, s}$. Observe agora que $l^{t}$ pode ser obtido por $l^{t}=\mathcal{J}^{t}\left(l^{t+1}\right)+g(t)$. Com isto, e lembrando que $l^{T}=g(T)$, 
é fácil mostrar que $l^{0}=\sum_{t=0}^{T} \mathcal{J}^{0, t-1}(g(t))$. Logo, temos que

$$
\mathcal{Z}^{T}=\left\langle l^{0}, \breve{\chi}^{0}\right\rangle \leq \overline{\mathcal{Z}}\left(\check{\chi}^{0}\right)
$$

independentemente de $T$. A partir disto, devemos então mostrar que $\left\|l^{0}\right\|$ é também limitado para qualquer horizonte de tempo. Para isto, considere uma base para o espaço $\mathcal{M}^{n, 1}$ formada pelas coleções de matrizes $s_{j}^{i} \in \mathcal{M}^{n, 1}, 0 \leq i \leq n$ e $0 \leq j \leq \eta$, em que $s_{j}^{i}$ apresenta o valor 1 na posição $i$ da matriz $j$ e zero nas demais. Para um horizonte de tempo finito, temos que $\left\|l^{0}\right\|<\infty$, de modo que é possível representar $l^{0} /\left\|l^{0}\right\|$ como combinação linear dos elementos $s_{j}^{i}$. Diante disso, utilizando (2.33), podemos escrever,

$$
\begin{aligned}
\left\|l^{0}\right\| & =\left\langle l^{0}, \frac{l^{0}}{\left\|l^{0}\right\|}\right\rangle=\left\langle l^{0}, \sum_{i=1}^{n} \sum_{j \in \mathcal{O}} \beta_{i, j} s_{j}^{i}\right\rangle=\sum_{i=1}^{n} \sum_{j \in \mathcal{O}} \beta_{i, j}\left\langle l^{0}, s_{j}^{i}\right\rangle \\
& \leq \sum_{i=1}^{n} \sum_{j \in \mathcal{O}}\left\langle l^{0}, s_{j}^{i}\right\rangle \leq \sum_{i=1}^{n} \sum_{j \in \mathcal{O}} \overline{\mathcal{Z}}\left(s_{j}^{i}\right) \leq \eta n \overline{\mathcal{Z}}^{+},
\end{aligned}
$$

$\forall T>0$, em que $\overline{\mathcal{Z}}^{+} \triangleq \max _{i, j} \overline{\mathcal{Z}}\left(s_{j}^{i}\right)$ e $\left|\beta_{i, j}\right| \leq 1$ para $0 \leq i \leq n$ e $0 \leq j \leq \eta$. Logo, obtemos um limitante para $\left\|l^{0}\right\|$ independente de $T$ e, portanto,

$$
\lim \sup _{T \rightarrow \infty}\left[\frac{\left\|l_{i}^{0}\right\|}{T+1}\right]=0, \forall i \in \mathcal{O} .
$$

Lema 2.4.5. Seja $\lambda^{t} \in \mathcal{M}^{1,1}$ definido para $0 \leq t \leq T$ e dado por $\lambda^{t}=\mathcal{E}^{t}\left(\lambda^{t+1}\right)+f(t)$, com $\lambda_{i}^{T} \in \mathbb{R}, \forall i \in \mathcal{O}$, em que $f(t) \in \mathcal{M}^{1,1}$ é uniformemente limitada, ou seja, $\exists m_{f}$ : $\left|f_{i}(t)\right| \leq m_{f}, \forall i \in \mathcal{O}$ e $t \geq 0$. Então, é fato que,

$$
\lim \sup _{T \rightarrow \infty}\left[\frac{\lambda_{i}^{0}}{T+1}\right] \leq m_{f}<\infty, \forall i \in \mathcal{O}
$$

Demonstração: Definindo $\hat{\lambda}^{t} \triangleq \hat{\phi}\left(\lambda^{t}\right)$ e $\hat{f}(t) \triangleq \hat{\phi}(f(t))$, podemos escrever,

$$
\hat{\lambda}^{t}=\mathbb{P}(t) \hat{\lambda}^{t+1}+\hat{f}(t)
$$

que nos leva à seguinte equação,

$$
\hat{\lambda}^{t}=\prod_{k=t}^{T-1} \mathbb{P}(k) \hat{\lambda}^{T}+\sum_{j=t}^{T-2} \prod_{k=t}^{j} \mathbb{P}(k) \hat{f}(j+1)+\hat{f}(t) .
$$


Definindo $\overline{\mathbb{P}}^{t, j} \triangleq \prod_{k=t}^{j} \mathbb{P}(k)$ e particularizando o resultado anterior para $t=0$, obtemos,

$$
\hat{\lambda}^{0}=\overline{\mathbb{P}}^{0, T-1} \hat{\lambda}^{T}+\sum_{j=0}^{T-2} \overline{\mathbb{P}}^{0, j} \hat{f}(j+1)+\hat{f}(0) .
$$

Utilizando a norma induzida $\|.\|_{\infty}$, definida como $\|G\|_{\infty}=\max _{i=1, \ldots, m} \sum_{j=1}^{n}\left|[G]_{i, j}\right|$, para $G \in \mathbb{R}^{m, n}$, em que |.| é definido como o valor absoluto, então, é verdade que,

$$
\begin{aligned}
\left\|\hat{\lambda}^{0}\right\|_{\infty} & =\left\|\overline{\mathbb{P}}^{0, T-1} \hat{\lambda}^{T}+\sum_{j=0}^{T-2} \overline{\mathbb{P}}^{0, j} \hat{f}(j+1)+\hat{f}(0)\right\|_{\infty} \\
& \leq\left\|\overline{\mathbb{P}}^{0, T-1}\right\|_{\infty}\left\|\hat{\lambda}^{T}\right\|_{\infty}+\sum_{j=0}^{T-2}\left\|\overline{\mathbb{P}}^{0, j}\right\|_{\infty}\|\hat{f}(j+1)\|_{\infty}+\|\hat{f}(0)\|_{\infty} .
\end{aligned}
$$

Como sabemos, o produto de matrizes de probabilidade de transição é também uma matriz de mesma classe, consequentemente, $\left\|\overline{\mathbb{P}}^{0, j}\right\|_{\infty}=1$, para $0 \leq j \leq T-1$. Com isto, obtemos,

$$
\left\|\hat{\lambda}^{0}\right\|_{\infty} \leq\left\|\hat{\lambda}^{T}\right\|_{\infty}+\sum_{j=0}^{T-1}\|\hat{f}(j)\|_{\infty} \leq\left\|\hat{\lambda}^{T}\right\|_{\infty}+T m_{f}
$$

Então,

$$
\lim \sup _{T \rightarrow \infty}\left[\frac{1}{T+1}\left\|\hat{\lambda}^{0}\right\|_{\infty}\right] \leq m_{f}
$$

e, portanto,

$$
\lim \sup _{T \rightarrow \infty}\left[\frac{\lambda_{i}^{0}}{T+1}\right] \leq m_{f}<\infty, \forall i \in \mathcal{O}
$$

Teorema 2.4.6. Seja o sistema $(\Psi, \mathcal{W})$ uniformemente detetável e $\breve{\mathcal{W}}$ uniformemente limitado. Considere ainda que o sistema $\Psi$ é controlado por $u^{*}(t)$, definido no Teorema 2.2.1. Então, o custo (2.18) existe e é dado por,

$$
\overline{\mathcal{W}}(\theta(0), x(0))=\lim \sup _{T \rightarrow \infty} \frac{1}{T+1}\left[w_{\theta(0)}^{0}\right]<\infty
$$

para qualquer $\theta(0) \in \mathcal{O}$ e $x(0) \in \mathbb{R}^{n}$.

Demonstração: Considere o custo em (2.31) e observe que a prova pode ser obtida avaliando-se o limite de cada termo entre chaves. Para o primeiro termo à esquerda, a prova por ser obtida por contradição. Assim, considere que $\left\|V^{0}\right\|>\alpha$. Então, da 
definição (2.2), temos que $\exists j \in \mathcal{O}$ tal que $\operatorname{tr}\left(V_{j}^{0} V_{j}^{0 \prime}\right) \geq \alpha / \eta$. Por outro lado, sabemos que existe um elemento $y \in \mathbb{R}^{n}$, que atende a $\|y\|=1$, tal que $y^{\prime} V_{j}^{0} y=\sigma^{*}\left(V_{j}^{0}\right)$, em que $\sigma^{*}\left(V_{j}^{0}\right)$ representa o maior valor singular de $V_{j}^{0}$. Definindo agora $\breve{\mathcal{X}}^{0}$ como $\breve{\mathcal{X}}_{j}^{0}=y y^{\prime}$ e $\breve{\mathcal{X}}_{i}^{0}=0_{n \times n}$ para $i \neq j$ obtemos $\left\|\breve{\mathcal{X}}^{0}\right\|=1$ e podemos escrever

$$
\left\langle V^{0}, \breve{\mathcal{X}}^{0}\right\rangle=\operatorname{tr}\left(V_{j}^{0} y y^{\prime}\right)=\sigma^{*}\left(V_{j}^{0}\right) \geq\left(\frac{\operatorname{tr}\left(V_{j}^{0} V_{j}^{0 \prime}\right)}{n}\right)^{\frac{1}{2}} \geq\left(\frac{\alpha}{\eta n}\right)^{\frac{1}{2}}\left\|\breve{\mathcal{X}}^{0}\right\| .
$$

Logo, para $\alpha \geq \eta n\left(\breve{\mathcal{W}}^{+}\right)^{2}$ obtemos $\left\langle V^{0}, \breve{\mathcal{X}}^{0}\right\rangle \geq \breve{\mathcal{W}}^{+}\left\|\breve{\mathcal{X}}^{0}\right\|$, o que contradiz a hipótese de que o custo $\breve{\mathcal{W}}$ é uniformemente limitado. Então, podemos afirmar que $\left\|V^{0}\right\|$ possui um limitante superior independente de $T$. Com isto, é possível concluir que

$$
\lim \sup _{T \rightarrow \infty} \frac{1}{T+1}\left[x(0)^{\prime} V_{\theta(0)}^{0} x(0)\right]=0, \quad \forall \theta(0) \in \mathcal{O} \text { e } x(0) \in \mathbb{R}^{n}
$$

Com relação ao termo seguinte, note que definindo $g(t) \triangleq 2\left(A(t)+B(t) K^{t}\right)^{\prime} \mathcal{E}^{t}\left(V^{t}\right) e(t)-$ $2\left(K^{t}\right)^{\prime} R(t) \bar{u}(t)-2 Q(t) \bar{x}(t)$, com $g(T)=-2 F(T) \bar{x}(T)$, é possível reescrever (2.14) como $v^{t}=\left(A(t)+B(t) K^{t}\right)^{\prime} \mathcal{E}^{t}\left(v^{t+1}\right)+g(t)$. Observe então que os termos que compõem $g(t)$ são uniformemente limitados, logo, podemos utilizar o Lema 2.4.4 para concluir

$$
\lim \sup _{T \rightarrow \infty} \frac{1}{T+1}\left[x(0)^{\prime} v_{\theta(0)}^{0}\right]=0, \quad \forall \theta(0) \in \mathcal{O} \text { e } x(0) \in \mathbb{R}^{n}
$$

Finalmente, para avaliar o último termo, observe que podemos definir $f(t) \triangleq \bar{x}(t)^{\prime} Q(t) \bar{x}(t)+$ $\bar{u}^{\prime}(t) R(t) \bar{u}(t)+e^{\prime}(t) \mathcal{E}^{t}\left(V^{t+1}\right) e(t)+\mathcal{E}^{t}\left(v^{t+1}\right)^{\prime} e(t)-\left(r^{t}\right)^{\prime} \Lambda^{t+1} r^{t}+\operatorname{tr}\left[H(t) H(t)^{\prime} \mathcal{E}^{t}\left(V^{t+1}\right)\right]$, com $f(T)=\bar{x}^{\prime}(T) F(T) \bar{x}(T)$, e reescrever (2.15) como $w^{t}=\mathcal{E}^{t}\left(w^{t+1}\right)+f(t)$. Mais uma vez, note que todos os termos que compõem $f(t)$ são uniformemente limitados, então, aplicando o Lema (2.4.5), podemos concluir que,

$$
\lim \sup _{T \rightarrow \infty} \frac{1}{T+1}\left[w_{\theta(0)}^{0}\right]<\infty, \quad \forall \theta(0) \in \mathcal{O}
$$

Portanto, o custo (2.18) existe e é dado por,

$$
\overline{\mathcal{W}}(\theta(0), x(0))=\lim \sup _{T \rightarrow \infty} \frac{1}{T+1}\left[w_{\theta(0)}^{0}\right]<\infty, \quad \forall \theta(0) \in \mathcal{O} \text { e } x(0) \in \mathbb{R}^{n}
$$




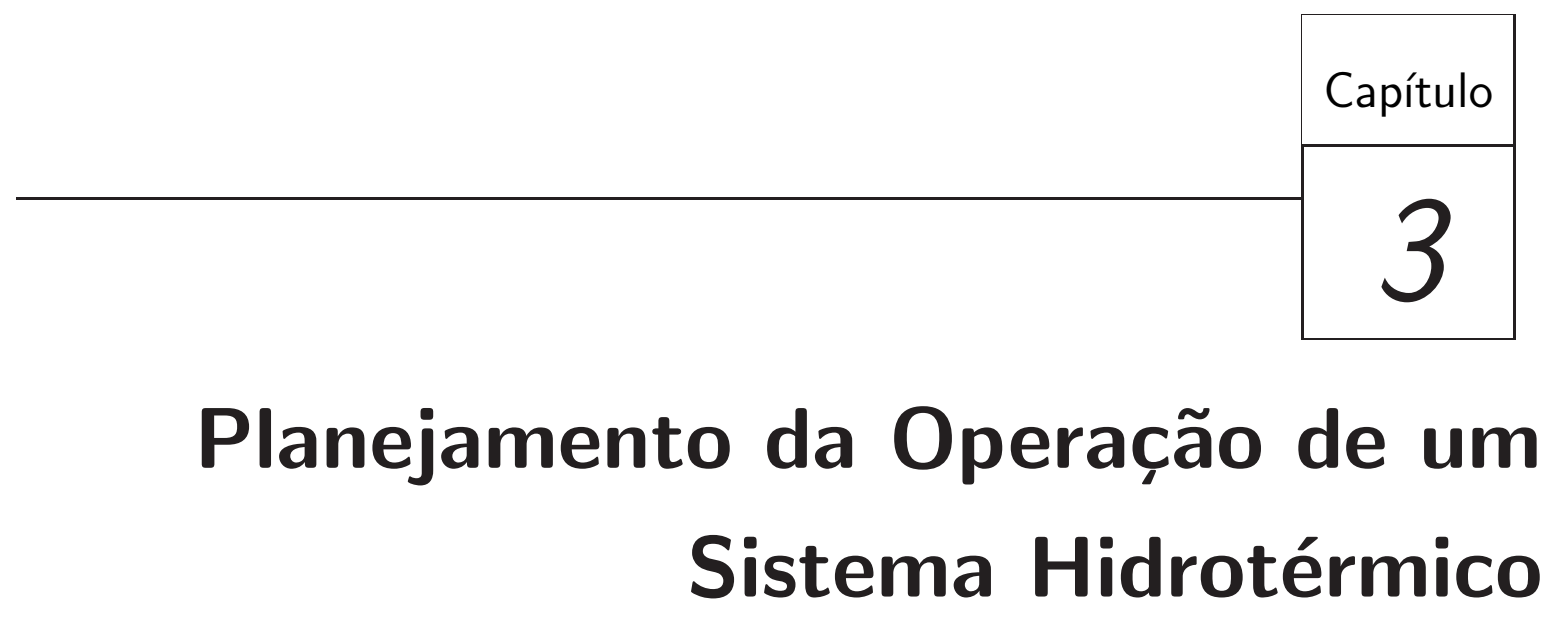

Neste capítulo apresentaremos os detalhes da implementação do RLQ com CMLP no planejamento da operação de um sistema hidrotérmico. Na Seção 3.1 apresentaremos os elementos fundamentais que definem este problema, como também os dois casos de estudo considerados. Nesta mesma seção, definiremos o problema solucionado pela PDE e as simplificações necessárias para a aplicação da PDD e do RLQ no planejamento hidrotérmico. Já na Seção 3.2 apresentaremos as principais características das usinas do rio São Francisco que compõem os casos de estudos. Além disto, revelaremos os detalhes da implementação dos controladores e das simulações realizadas. Na Seção 3.3 discutiremos e apresentaremos os resultados obtidos pelo RLQ em comparação com as técnicas PDE e PDD.

\subsection{Definição do Problema}

Para alcançar um modelo representativo de um conjunto de usinas é necessário compreender as principais características de um reservatório hidroelétrico, como podemos observar na Figura 3.1. Nesta figura, as setas azuis indicam as rotas do fluxo d'água. A água que entra no reservatório, denominada vazão afluente, é proveniente 
de defluências das usinas a montante e de uma certa vazão oriunda, principalmente, de afluentes que desembocam entre o reservatório e as usinas imediatamente à montante, denominada vazão incremental. A vazão incremental é a principal fonte de incerteza a ser considerada no gerenciamento de um reservatório. Uma vez armazenada, a água pode ser consumida, turbinada, vertida ou evaporar-se. Nesta figura, também estão apresentados os níveis máximo e mínimo, que definem as restrições operativas quanto aos volumes máximo e mínimo que podem ser armazenados e também o denominado volume útil. Uma parcela deste volume, denominada volume de espera, deve ser mantida vazia durante a época de chuva para promover o controle de cheias na bacia. Como resultado, é possível acomodar as afluências excessivas que porventura ocorram neste período e manter as defluências em níveis seguros. Na figura, podemos observar ainda os elementos que definem a potência gerada, são eles, a quantidade de água turbinada, a altura de queda bruta e o coeficiente de produtividade que é um índice relativo à eficiência dos geradores, turbinas e circuito hidráulico da barragem.

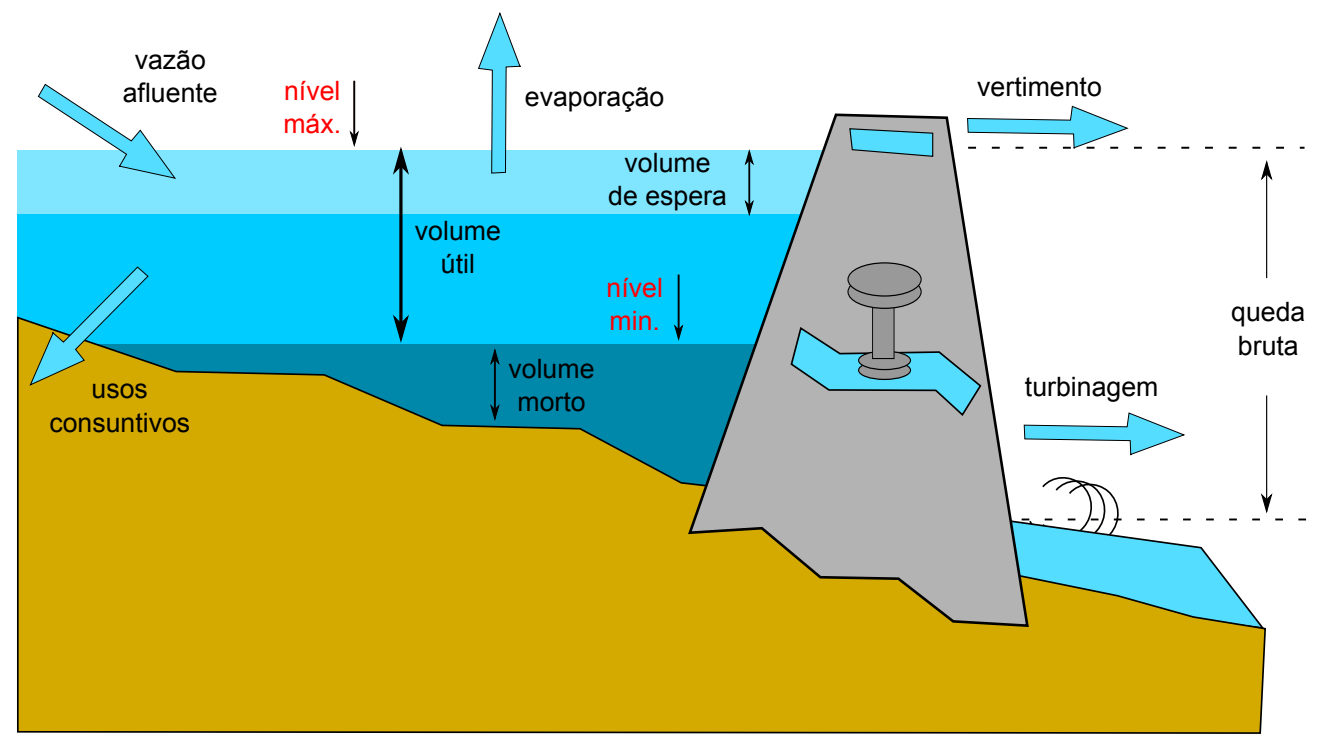

Figura 3.1: Principais características de uma usina hidroelétrica.

Diante do exposto, desprezando os usos consuntivos, podemos representar o balanço hídrico do $i$-ésimo reservatório de um sistema de usinas, pela equação a seguir,

$$
[x(t+1)]_{i}=[x(t)]_{i}-[r(t)]_{i}+\sum_{j \in \Omega_{i}}[r(t)]_{j}+[v(t)]_{i}-[l(t)]_{i}
$$

com $[x(0)]_{i}=x_{0, i}$; sendo $[x(t)]_{i}$ o volume armazenado de água no reservatório $i$ no início do $t$-ésimo mês, $[r(t)]_{i}$ o volume deplecionado, isto é, a soma do volume vertido 
e turbinado durante o intervalo $t, \Omega_{i}$ o conjunto de usinas imediatamente à montante do reservatório $i$, ou seja, cujos volumes deplecionados seguem diretamente para o reservatório $i,[v(t)]_{i}$ o volume incremental de água em cada reservatório e, por fim, $[l(t)]_{i}$ o volume de água perdido por evaporação.

O vertimento ocorre para manter os níveis dos reservatórios abaixo do nível máximo de operação, ou, equivalentemente, $[x(t)]_{i} \leq\left[x^{\max }(t)\right]_{i}$, de modo que pode ser dado por

$$
[s(t)]_{i}=\max \left(0,[x(t)]_{i}-c_{v}[u(t)]_{i}+\sum_{j \in \Omega_{i}}[r(t)]_{j}+[v(t)]_{i}-[l(t)]_{i}-\left[x^{\max }(t)\right]_{i}\right)
$$

em que $[u(t)]_{i}$ é a vazão turbinada pela usina $i$ e $c_{v}$ é uma constante necessária para converter unidade de vazão em volume. Assim, o deplecionamento desta usina pode ser obtido fazendo $[r(t)]_{i}=c_{v}[u(t)]_{i}+[s(t)]_{i}$.

O volume evaporado pode ser calculado por,

$$
[l(t)]_{i}=c_{e} h_{i}(t)\left[\frac{a_{i}\left([x(t)]_{i}\right)+a_{i}\left([x(t+1)]_{i}\right)}{2}\right]
$$

cujo o parâmetro $h_{i}(t)$ refere-se à taxa média mensal de evaporação líquida correspondente ao mês $t$, a função $a_{i}($.$) fornece a área do espelho d'água em função do volume do$ reservatório e a constante $c_{e}$ converte valores expressos em $\mathrm{mm}$ para volume. No Brasil, a área do espelho d'água é calculada pela combinação de dois polinômios de quarto grau que fornecem a cota do reservatório em relação ao seu volume, função $\varphi_{i}($.$) apre-$ sentada abaixo, e a área pela cota, dada pela função $\rho_{i}($.$) . Em geral, assim como neste$ trabalho, assume-se que a área de cada reservatório relaciona-se linearmente com o seu volume, de modo que é possível representar $a_{i}($.) como um polinômio de primeiro grau.

Para um sistema com $N$ reservatórios, utilizando uma cadeia de Markov para descrever as vazões incrementais, é possível reescrever a equação (3.1) como o seguinte,

$$
\Psi_{v}:\left\{\begin{array}{l}
x(t+1)=A(t) x(t)+B(t) u(t)+e_{\theta(t)}(t)+C(t) s(t) \\
x(0)=x_{0} \in \mathbb{R}^{N}
\end{array}\right.
$$

sendo $x(t) \in \mathbb{R}^{N}$, o vetor de estados que representa o volume armazenado de água em cada reservatório no início do intervalo $t, u(t) \in \mathbb{R}^{M}$, o vetor controle, que indica a vazão turbinada para os mesmos reservatórios durante o período $t, e_{\theta(t)}(t)$, o vetor de entradas exógenas relacionado com o vazão incremental e a evaporação, $A(t)$, a matriz ligada à evaporação, e, $B(t)$ e $C(t)$, matrizes relacionadas, principalmente, com a rota do fluxo de água dentro do sistema. A variável $\theta(t)$ representa os valores 
obtidos pela realização de uma cadeia de Markov previamente conhecida, representada por uma matriz de probabilidade de transição, $\mathbb{P}_{v}(t)$. Os detalhes para a construção deste modelo estão apresentados no Apêndice B.1. Para os propósitos deste trabalho, consideramos ainda duas versões simplificadas do sistema $\Psi_{v}$, definidas por $\Psi_{d}$ e $\Psi_{R L Q}$. Definimos $\Psi_{d}$ assumindo que as vazões incrementais de cada reservatório são dadas por suas médias mensais históricas. Para definir $\Psi_{R L Q}$, desconsideramos os vertimentos, assim, adotamos $s(t)=0$ para todo $t \geq 0$ em $\Psi_{v}$.

Como mencionamos, utilizamos a função objetivo abaixo, relacionada com os custos hidrotérmicos [4], definida por,

$$
\mathcal{H}_{u}^{t^{\prime}, T}\left(x_{0}\right)=\sum_{t=t^{\prime}}^{T-1}\left[D(t)-\sum_{i=1}^{N} P_{i}(t)\right]^{2}
$$

para $0 \leq t^{\prime} \leq T-1$, sendo $D(t)$ a demanda de potência a ser atendida no período $t$ e $P_{i}(t)$ a potência gerada pela usina $i$ no mesmo período de tempo. A potência gerada por cada usina pode ser calculada pela seguinte expressão

$$
P_{i}(t)=\kappa_{i}\left(\varphi_{i}\left([x(t)]_{i}\right)-\mu_{i}\left([r(t)]_{i}\right)\right)[u(t)]_{i}
$$

em que $\kappa_{i}$ é o coeficiente de produtibilidade da $i$-ésima usina, $\mu_{i}($.) é um polinômio de quarto grau que representa o nível do canal de fuga versus depleção da usina $i$. Note que a diferença entre os polinômios entre parênteses definem a queda bruta, indicada na Figura 3.1.

Considerando os elementos acima, uma forma de tratar o planejamento da operação hidrotérmica é solucionando o seguinte problema de otimização,

$$
\begin{aligned}
\mathbf{P}_{\mathbf{v}}^{\mathcal{H}}: \quad \mathcal{H}^{T}= & \inf _{u} E\left\{\mathcal{H}_{u}^{0, T}\left(x_{0}\right)\right\} \\
\text { s.a. } \quad & \Psi_{v} e(3.2) \\
& {[x(t)]_{i} \geq\left[x^{\min }(t)\right]_{i} } \\
& {\left[u^{\min }(t)\right]_{i} \leq[u(t)]_{i} \leq\left[u^{\max }(t)\right]_{i} } \\
& i=1, \ldots, N,
\end{aligned}
$$

em que $\mathcal{H}^{T}$ é definido como o custo ótimo para o problema e os termos $\left[x^{\min }(t)\right]_{i}$, $\left[u^{\min }(t)\right]_{i}$ e $\left[u^{\max }(t)\right]_{i}$ são grandezas que refletem os limites de operação dos reservatórios com relação aos volumes e turbinagens. Um segundo modo de tratar o problema, é considerar um cenário mais simples em que as vazões são determinísticas. Assim, definimos também $\mathbf{P}_{\mathbf{d}}^{\mathcal{H}}$ igual a $\mathbf{P}_{\mathbf{v}}^{\mathcal{H}}$, porém, sujeito à dinâmica $\Psi_{d}$ em lugar de $\Psi_{v}$. 
Ainda, desconsiderando as restrições para as variáveis de estado e controle, definimos um terceiro problema de otimização, denominado $\mathbf{P}_{\mathbf{v}}^{\mathcal{W}}$ e definido como segue

$$
\begin{gathered}
\mathbf{P}_{\mathbf{v}}^{\mathcal{W}}: \overline{\mathcal{W}}(\theta(0), x(0))=\inf _{u_{0}}\left[\lim \sup _{T \rightarrow \infty}\left\{\frac{1}{T+1} \mathcal{W}_{0}^{T}\left(\theta(0), x(0), u_{0}^{T-1}\right)\right\}\right] \\
\text { s.a. } \\
\Psi_{R L Q} .
\end{gathered}
$$

Lembrando que, para que a solução de $\mathbf{P}_{\mathbf{v}}^{\mathcal{W}}$ seja interessante para o planejamento hidrotérmico, devemos definir alvos e matrizes de peso que favoreçam a obtenção de baixos valores para $\mathcal{H}_{u}^{0, T}\left(x_{0}\right)$.

Para avaliar os controladores em um cenário distinto, criamos as dinâmicas $\check{\Psi}_{v}$,

$\check{\Psi}_{d}$ e $\check{\Psi}_{R L Q}$ semelhantemente a $\Psi_{v}, \Psi_{d}$ e $\Psi_{R L Q}$, porém, considerando nulo o efeito da evaporação. Valendo-se destas novas dinâmicas definimos os problemas $\check{\mathbf{P}}_{\mathbf{v}}^{\mathcal{H}}, \check{\mathbf{P}}_{\mathbf{d}}^{\mathcal{H}}$ e $\check{\mathbf{P}}_{\mathbf{v}}^{\mathcal{W}}$, de modo semelhante aos problemas recém apresentados. Definimos como cenário 1, os modelos apresentados em que se considera o efeito da evaporação, e cenário 2, os problemas recém apresentados, ou seja, que desprezam o efeito da evaporação no balanço hídrico. Destacamos ainda, que os problemas $\mathbf{P}_{\mathbf{v}}^{\mathcal{H}}$ e $\check{\mathbf{P}}_{\mathbf{v}}^{\mathcal{H}}$ foram solucionados via PDE, já as soluções para $\mathbf{P}_{\mathbf{d}}^{\mathcal{H}}$ e $\check{\mathbf{P}}_{\mathbf{d}}^{\mathcal{H}}$ foram obtidas com o uso da PDD e, por fim, utilizamos os resultados definidos pelo Teorema 2.2.1 para obter as soluções de $\mathbf{P}_{\mathbf{v}}^{\mathcal{W}}$ e $\check{\mathbf{P}}_{\mathbf{v}}^{\mathcal{W}}$.

\subsection{Métodos e Casos de Estudos}

A águas do rio São Francisco nascem na Serra da Canastra em Minas Gerais, percorrem boa parte dos estados da Bahia e Pernambuco e desembocam no Oceano Atlântico, na divisa dos Estados de Alagoas e Sergipe. Em seus 2700Km de extensão, o rio abriga seis usinas hidroelétricas que somam uma potência instalada de 10.330 GW [13]. Devido ao seu grande potencial hídrico e por atravessar uma região de baixa precipitação, o rio São Francisco tem sido alvo de fervorosos debates entre ambientalistas, comunidades ribeirinhas, políticos e até o capital internacional em torno da sua manutenção e utilização. Recentemente, com a implantação do Projeto de Integração do Rio São Francisco com Bacias Hidrográficas do Nordeste Setentrional (PISF), que visa construir dois canais de grande porte para a adução e transporte de parte de suas águas, este cenário se tornou ainda mais dramático, motivando novos estudos que dêem suporte a uma exploração cada vez mais sustentável e otimizada dos recursos deste rio.

O método proposto foi aplicado às suas seis usinas mais próximas da foz: Sobradinho, Itaparica, Moxotó, Paulo Afonso I-II-III, Paulo Afonso IV e Xingó. Estes 
reservatórios se encontram em áreas com altas temperatura e baixíssimos índices pluviométricos, de modo que o fenômeno da evaporação é uma variável importante a ser considerada em suas operações. Em consonância com o órgão gestor do sistema elétrico brasileiro, ONS (Operador Nacional do Sistema Elétrico), consideramos os reservatórios de Moxotó, Paulo Afonso 123 e Paulo Afonso 4, como um complexo único, que denominamos Complexo Moxotó [21]. A disposição dos reservatórios ao longo do rio e suas principais características estão apresentas na Figura 3.2 e Tabela 3.1.

Dos reservatórios citados, apenas Sobradinho e Itaparica possuem capacidade de regularização, ou seja, podem armazenar a vazão afluente para ser turbinada posteriormente, assim como a usina representada pela Figura 3.1. Os demais são operados à fio d'água, isto é, o volume de água que entra em seus reservatórios em um certo período é, necessariamente, turbinado ou vertido no mesmo intervalo de tempo. Logo, para estes reservatórios não cabe ação de controle em suas operações, de modo que suas turbinagens são determinadas diretamente pela defluência de Itaparica. Com isto, para facilitar a exposição, não apresentaremos detalhes sobre a operação destes reservatórios. Deste modo, representamos o sistema estudado com $N=2$ e $M=2$, na equação (3.4), e associamos os índices $i=1$ para Sobradinho e $i=2$ para Itaparica.

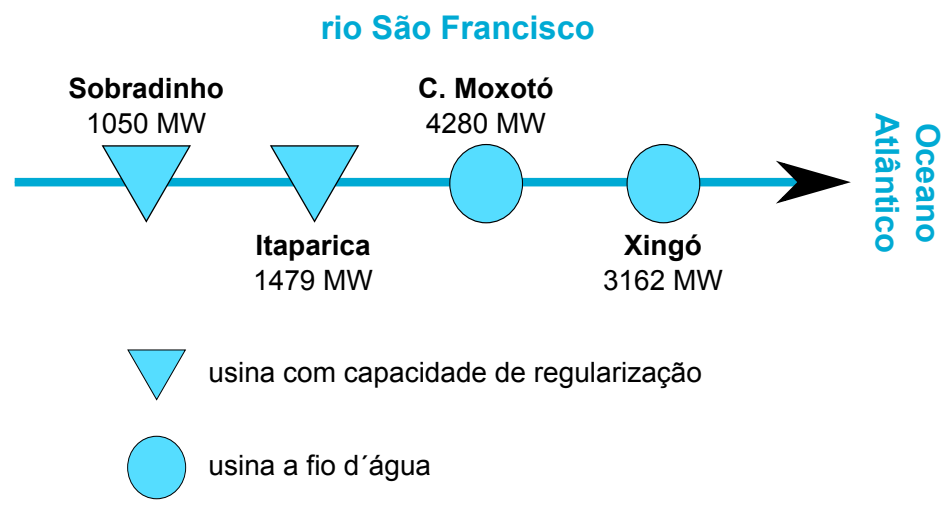

Figura 3.2: Esquema topológico das usinas do rio São Francisco.

As restrições de volume e turbinagem para estes reservatórios estão apresentadas na Tabela B.2 e podem ser obtidas diretamente do sítio eletrônico da companhia responsável pelos reservatórios, denominada Eletrobras Furnas. As restrições para o volume estão relacionadas às limitações de seus níveis operacionais (colunas 3 e 4 da Tabela 3.1) e foram utilizadas sem modificações neste trabalho. Da mesma maneira, utilizamos o engolimento máximo como restrição superior para a turbinagem dos reservatórios. Por outro lado, para garantir a obtenção da solução ótima para os problemas $\mathbf{P}_{\mathbf{v}}^{\mathcal{H}}$ e $\check{\mathbf{P}}_{\mathbf{v}}^{\mathcal{H}}$, diminuímos a restrição inferior das vazões defluentes de Sobradinho para o valor de 450 
$\mathrm{m}^{3} / \mathrm{s}$. Na prática, para garantir a navegabilidade a jusante deste reservatório, deve-se manter a vazão acima de $1300 \mathrm{~m}^{3} / \mathrm{s}$ [12]. Entretanto, este valor é maior que o menor valor registrado para as vazões afluentes de Sobradinho, como pode ser observado pela Figura B.1. Esta situação pode provocar soluções infactíveis e violação quanto aos limites mínimos de volume e/ou turbinagem, o que dificulta a garantia quanto a obtenção da solução ótima pela PDE. Pelos mesmo motivos ignoramos as restrições de defluência máxima e os volumes de espera.

Tabela 3.1: Principais características dos reservatórios estudados.

$\begin{array}{lccc} & \begin{array}{c}\text { Capacidade } \\ \text { Acumulação } \\ \left(\mathrm{hm}^{3}\right)\end{array} & \begin{array}{c}\text { Nível Máximo } \\ \text { Operacional } \\ (\mathrm{m})\end{array} & \begin{array}{c}\text { Nível Mínimo } \\ \text { Operacional }\end{array} \\ \text { Sobradinho } & 28,669 & 392,50 & (\mathrm{~m}) \\ \text { Itaparica } & 3,548 & 304,00 & 380.50 \\ \text { C. Moxotó } & - & 251.50 & 299.00 \\ \text { Xingó } & - & 138.00 & 251.50 \\ & & & 138.00\end{array}$

Como as médias das vazões incrementais de Itaparica, C. Moxotó e Xingó são muito pequenas, inferiores a 3\% da vazão afluente de Sobradinho, consideramos estas variáveis como dadas por suas médias históricas, pois, incluir novos modos para representálas no cenário estocástico inviabilizaria a utilização da PDE devido ao agravamento do "mal da dimensionalidade". Logo, as vazões afluentes de Sobradinho são a única fonte de incerteza considerada neste trabalho. Para obter $\mathbb{P}_{v}(t)$ e definir os valores referentes a cada modo da vazão de Sobradinho, empregamos os passos sugeridos por [17] considerando $\eta=10$. A proposta destes autores é dividir o intervalo entre as observações mínimas e máximas de cada mês em segmentos de tamanho variável, de modo que cada intervalo contenha o mesmo número de observações. Define-se então o valor característico de cada modo de acordo com a distribuição dos pontos dentro de cada intervalo. Utilizando uma quantidade suficiente de modos, os autores afirmaram que este método conserva a média e variância da série original, o que não é verificado no método tradicional, no qual se divide o intervalo de observações em segmentos de mesmo tamanho. A série de vazão utilizada foi obtida no sítio do próprio ONS [21] e se estende de Janeiro de 1931 a Dezembro de 2007, como nos mostra a Figura B.1 da Seção B.3 do Apêndice. Nesta mesma seção, apresentamos ainda os valores característicos e algumas das matrizes de probabilidade de transição obtidas.

Consideramos que a demanda de potência do sistema, necessária para o cálculo do custo (3.5), era $D(t)=10.1 \mathrm{GW}, \forall t \in[0, \ldots T-1]$, que se refere a um valor ligeiramente 
superior à potência máxima que pode ser produzida pelo conjunto de usinas neste caso de estudo. Os coeficientes dos polinômios e de produtibilidade das usinas, necessários ao cálculo das equações (3.3) e (3.6), estão apresentados no Apêndice pelas Tabelas B.2, B.4, B.5 e B.6. Os valores utilizados para $h_{i}(t)$ estão apresentados na Tabela B.3. Todos estes parâmetros foram obtidos diretamente do sítio eletrônico da Câmara de Comercialização de Energia Elétrica (CCEE).

\subsubsection{Simulações}

Para comparar o desempenho dos controladores, empregamos um conjunto de realizações para as vazões afluentes de Sobradinho. Para compreender este processo, considere $w$ como um elemento do espaço de probabilidade de $\mathbb{P}_{v}(t)$ de modo que $\theta(0, w), \ldots, \theta(T, w)$ é uma realização desta cadeia. Empregando simulação de Monte Carlo, encontramos $w_{1}^{1}, w_{1}^{2}, \ldots, w_{1}^{S_{1}}$, em que $S_{1}=3000$ é o número de realizações obtidas. Para cada, simulamos os controladores utilizando as restrições de $\mathbf{P}_{\mathbf{v}}^{\mathcal{H}}$, para o cenário 1, e $\check{\mathbf{P}}_{\mathbf{v}}^{\mathcal{H}}$, para o cenário 2. Para todas as variáveis aleatórias de interesse, definimos como seu valor esperado a média obtida considerando todas estas realizações, como exemplo, definimos $E\left\{\mathcal{H}_{u}^{0, T}\left(x_{0}\right)\right\}$ como o custo hidrotérmico esperado, dado pela média de $\left(S_{1}\right)^{-1} \sum_{j=1}^{S_{1}} \mathcal{H}_{u}^{0, T}\left(x_{0}, w_{1}^{j}\right)$. Além disto, para avaliar as características de longo prazo do RLQ e minimizar o impacto das condições iniciais e finais dos reservatórios, promovemos também uma segunda rodada de simulações, em que empregamos a série de vazão afluente real de Sobradinho, que compreende 924 meses (77 anos).

É importante mencionar que durante as simulações, a turbinagem dos controladores foi corrigida para que estes turbinassem o máximo possível antes de verter. Isto ocorre quando é necessário o vertimento e a turbinagem sugerida pelo controlador é menor que a turbinagem máxima. Do mesmo modo, restringimos os controles que determinavam valores para a turbinagem que levariam à violação das restrições de limite mínimo. Lembrando que o valor adotado para a restrição inferior da turbinagem de Sobradinho, $450 \mathrm{~m}^{3} / \mathrm{s}$, permite que esta estratégia seja sempre possível.

Em todas as simulações definimos um horizonte de tempo de 5 anos, ou seja, adotamos $T=60$, como sugerido para o planejamento de longo prazo [24]. Em todo o trabalho, definimos o mês de Maio para iniciar o horizonte de tempo, pois, neste mês se inicia a época de seca na região das usinas [5]. Esta é uma prática comum tendo em vista que as vazões futuras podem ser melhor previstas no final da época de chuvas [29]. Os volumes iniciais dos reservatórios de Sobradinho e Itaparica foram definidos como o valor esperado para o mês de Maio quando as soluções de $\mathbf{P}_{\mathbf{d}}^{\mathcal{H}}$ e $\check{\mathbf{P}}_{\mathbf{d}}^{\mathcal{H}}$ são simuladas utilizando as restrições de $\mathbf{P}_{\mathbf{v}}^{\mathcal{H}}$. Assim, utilizamos $[x(0)]_{1}=24000 \mathrm{hm}^{3}$ e $[x(0)]_{2}=10660$ 
$h \mathrm{~m}^{3}$ no cenário 1 , e $[x(0)]_{1}=25500 \mathrm{hm}^{3}$ e $[x(0)]_{2}=10782 \mathrm{hm}^{3}$, para o segundo cenário. Para o sorteio de $\theta(0)$, utilizamos a distribuição de probabilidade inicial dada por $\mu(0)=\left[\prod_{z=0}^{11} \mathbb{P}_{v}(z)\right]^{\prime}[1 / \eta 1 / \eta \cdots 1 / \eta]^{\prime}$.

\subsubsection{Soluções para $\mathbf{P}_{\mathrm{d}}^{\mathcal{H}}, \check{\mathbf{P}}_{\mathrm{d}}^{\mathcal{H}}, \mathbf{P}_{\mathrm{v}}^{\mathcal{H}} \mathbf{e} \check{\mathbf{P}}_{\mathrm{v}}^{\mathcal{H}}$}

Basicamente, a proposta da programação dinâmica é fragmentar o problema original em sub-problemas cujas soluções podem ser obtidas mais facilmente. Entretanto, para os problemas em questão, não é possível obter soluções analíticas como a apresentada no Teorema 2.2.1. Assim, utilizamos a equação de Bellman, reversamente no tempo, considerando os elementos dos conjuntos $X_{i}(t)$ e $U_{i}(t)$, para $i=1$, 2 e $0 \leq t \leq T-1$, obtidos pela discretização uniforme dos intervalos definidos pelos limites superiores e inferiores das restrições de volume e turbinagem. Para cada elemento de $X_{i}(t)$ avaliamos o custo de continuação incorrido por todas as ações pertencentes a $U_{i}(t)$. Contudo, em geral, ações de controle aplicadas em elementos de $X_{i}(t)$ levam o sistema a pontos não pertencentes a $X_{i}(t+1)$, de modo que utilizamos interpolação linear para calcular os custos futuros de cada ação. O controle que promovia o menor custo de continuação era definido como ótimo para o elemento de $X(t)$ considerado. Para detalhes sobre o método e discussão sobre diferentes formas de implementação da programação dinâmica no gerenciamento de reservatórios, consulte [20], e, para excelente revisão sobre o tema, consulte [36].

Para garantir a convergência da PDE e PDD, aplicamos o método acima para um horizonte de 108 meses e descartamos os últimos 48 meses. Estes valores foram definidos pela observação das trajetórias do estado e controle para $\mathbf{P}_{\mathbf{d}}^{\mathcal{H}}$ e $\check{\mathbf{P}}_{\mathbf{d}}^{\mathcal{H}}$, que se mostraram claramente convergentes para horizontes maiores que 24 meses. É interessante ressaltar que estes resultados estão de acordo com [3], em que os autores obtiveram convergência da Programação Dinâmica após 30 meses.

Quanto mais pontos adicionamos aos conjuntos $X(t)$ e $U(t)$, obtemos uma solução mais próxima da solução ótima, porém aumentamos também os custos computacionais do método, de modo que se torna interessante encontrar uma quantidade de pontos que promova boa solução aliada a baixos custos computacionais. Assim, considere $n_{p}$ como o número de pontos utilizados para compor $X(t)$ e $U(t)$. Para definir este valor, avaliamos o custo ótimo de $\mathbf{P}_{\mathbf{d}}^{\mathcal{H}}$ para $n_{p}$ crescente. Dado o enorme volume do reservatório de Sobradinho, utilizamos $2 n_{p}$ pontos para discretizar esta variável. Os resultados obtidos estão apresentados na Figura 3.3. Note que, a partir de 30 pontos, os custos se apresentam semelhantes. A partir desta análise, definimos $n_{p}=50$ para obter a 
solução dos problemas $\mathbf{P}_{\mathbf{d}}^{\mathcal{H}}, \mathbf{P}_{\mathbf{v}}^{\mathcal{H}}, \check{\mathbf{P}}_{\mathbf{d}}^{\mathcal{H}}$ e $\check{\mathbf{P}}_{\mathbf{v}}^{\mathcal{H}}$. Vale observar que esta quantidade de pontos está muito acima dos 11 pontos utilizados por [2] para a programação dinâmica.

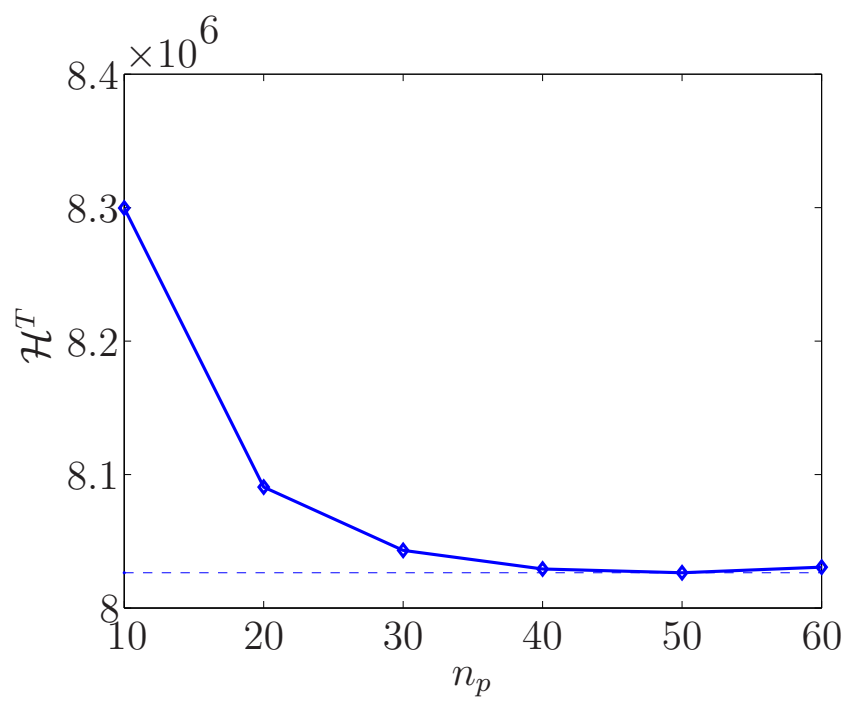

Figura 3.3: Custo ótimo de $\mathbf{P}_{\mathbf{d}}^{\mathcal{H}}$ para diferentes $n_{p}$.

\subsubsection{Soluções para $\mathbf{P}_{\mathrm{v}}^{\mathcal{W}}$ e $\check{\mathbf{P}}_{\mathrm{v}}^{\mathcal{W}}$}

Para obter o RLQ aplicado no planejamento da operação do sistema hidrotérmico, utilizamos o Teorema 2.2.1 com o auxílio das soluções de $\mathbf{P}_{\mathbf{d}}^{\mathcal{H}}$ e $\check{\mathbf{P}}_{\mathbf{d}}^{\mathcal{H}}$, que denominamos $u^{d}(.,$.$) e \check{u}^{d}(.,$.$) , nesta ordem. Para definir \bar{u}_{i}(t)$ em ambos os cenários, utilizamos os valores obtidos por $u^{d}(.,$.$) e \check{u}^{d}(.,$.$) , para 0 \leq t \leq T-1$, quando estes foram simulados com as mesmas restrições de $\mathbf{P}_{\mathbf{d}}^{\mathcal{H}}$ e $\check{\mathbf{P}}_{\mathbf{d}}^{\mathcal{H}}$. A trajetória de estado associada foi definida como os alvos para o estado. As curvas guia obtidas para o volume de Sobradinho e Itaparica podem ser observadas pelas curvas tracejadas nas figuras 3.8 e B.6, já os alvos para a turbinagem destes reservatórios estão apresentados pelas figuras B.4 e B.4.

Assim como os trabalhos [34, 23], consideramos $Q_{i}(t)$ e $R_{i}(t)$ como matrizes diagonais. Para obter tais matrizes, empregamos simulação de Monte Carlo para obter as realizações $w_{2}^{1}, w_{2}^{2}, \ldots, w_{2}^{S_{2}}$, em que $S_{2}=1000$ é o número de realizações obtidas. Após, simulamos $u^{d}(.,$.$) e \check{u}^{d}(.,$.$) utilizando as restrições de \mathbf{P}_{\mathbf{v}}^{\mathcal{H}}$ e $\check{\mathbf{P}}_{\mathbf{v}}^{\mathcal{H}}$, respectivamente. O objetivo deste método é fazer com que o RLQ mimetize o comportamento destes controladores em relação às curvas guia de cada cenário. Assim, para cada $w_{2}^{j}$, com $1 \leq j \leq S_{2}$, obtivemos as trajetórias de estado $\left[x^{d}\left(t, w_{2}^{j}\right)\right]_{i}$ e $\left[\check{x}^{d}\left(t, w_{2}^{j}\right)\right]_{i}$, e os valores de controle $\left[u^{d}\left(t, w_{2}^{j}\right)\right]_{i}$ e $\left[\check{u}^{d}\left(t, w_{2}^{j}\right)\right]_{i}$, referentes à usina $i$. Para que o RLQ ofereça soluções similares à PDD, pretendemos, para o cenário 1 por exemplo, atribuir valores relativamente 
grandes para $[Q(t)]_{i i}$ ou $[R(t)]_{i i}$ quando os valores de $\left[x^{d}\left(t, w_{2}^{j}\right)\right]_{i}$ ou $\left[u^{d}\left(t, x^{d}\left(t, w_{2}^{j}\right)\right)\right]_{i}$, aparecem próximos à $\bar{x}_{i}(t)$ ou $\bar{u}_{i}(t)$. Entretanto, empregamos de fato as realizações $\left[\underline{x}^{d}\left(t, w_{2}^{j}\right)\right]_{i}$ dadas por $\underline{x}^{d}\left(t+1, w_{2}^{j}\right)=A(t) x^{d}\left(t, w_{2}^{j}\right)+B(t) u^{d}\left(t, x^{d}\left(t, w_{2}^{j}\right)\right)+e_{\theta\left(t, w_{2}^{j}\right)}(t)$. Somente após, encontramos $\underline{x}^{d}\left(t+1, w_{2}^{j}\right)+C(t) s\left(t, \underline{x}^{d}\left(t+1, w_{2}^{j}\right)\right)$ e utilizamos as demais restrições para obter o estado real do sistema durante a simulação, ou seja, $x^{d}\left(t+1, w_{2}^{j}\right)$. Esta estratégia se fez necessária pois as curvas guia obtidas para o volume se aproximam dos limites inferiores (cenário 1) e superiores (cenário 2) dos reservatórios em determinados meses, levando a uma diminuição do desvio padrão de $x^{d}\left(t+1\right.$, w $\left.w_{2}^{j}\right)$ nestes pontos e, consequentemente, à matrizes de ponderação com valores muito elevados. Assim, definimos

$$
[Q(t)]_{i i}=\sum_{t=0}^{T}(T+1)^{-1} \sum_{j=1}^{S_{2}} S_{2}^{-1}\left(\left[\underline{x}^{d}\left(t, w_{2}^{j}\right)\right]_{i}-\bar{x}(t)\right)^{-2}
$$

e

$$
[R(t)]_{i i}=\sum_{t=0}^{T-1} T^{-1} \sum_{j=1}^{S_{2}} S_{2}^{-1}\left(\left[u^{d}\left(t, x^{d}\left(t, w_{2}^{j}\right)\right]_{i}\right)-\bar{u}(t)\right)^{-2}
$$

para $0 \leq t \leq T$ e $i=1,2$. Como atribuímos valores idênticos para $\bar{x}_{i}(t)$ e $\bar{u}_{i}(t)$, para todo $i \in \mathcal{O}$, abusamos da notação nas equações acima ao omitirmos o índice $i$. Note que utilizando estas equações, tornamos adimensionais os funcionais $p(t)$ e $q(t)$, definidos na Seção 2.2, e estabelecemos, de certa forma, pesos condizentes com a magnitude de cada variável. Os pesos obtidos para ambos os cenários estão apresentados na Tabela 3.2 .

Tabela 3.2: Pesos utilizados neste trabalho $(\forall i \in \mathcal{O}$ e $t \geq 0)$.

$\begin{array}{lrr} & \text { Cenário 1 } & \text { Cenário 2 } \\ {\left[Q_{i}(t)\right]_{11}} & 1.9338 \times 10^{-8} & 1.2391 \times 10^{-8} \\ {\left[Q_{i}(t)\right]_{22}} & 1.5749 \times 10^{-6} & 3.1811 \times 10^{-6} \\ {\left[R_{i}(t)\right]_{11}} & 4.0444 \times 10^{-6} & 4.0381 \times 10^{-6} \\ {\left[R_{i}(t)\right]_{22}} & 9.1746 \times 10^{-6} & 7.0130 \times 10^{-6}\end{array}$

Com o intuito de verificar a possibilidade de obtermos menores custos hidrotérmicos para o RLQ, investigamos também o desempenho deste controlador para diferentes pesos. Para isto, a partir dos valores dispostos na Tabela 3.2, criamos de forma semelhante 4 conjuntos com 7 elementos cada referentes a $\left[Q_{i}(t)\right]_{11},\left[Q_{i}(t)\right]_{22},\left[R_{i}(t)\right]_{11}$ e $\left[R_{i}(t)\right]_{22}$. Por exemplo, para ponderar o desvio do volume de Sobradinho, avaliamos 7 pontos linearmente espaçados no intervalo $0.50 \times\left[Q_{i}(t)\right]_{11}$ e $1.50 \times\left[Q_{i}(t)\right]_{11}$, incluindo 
os extremos. Após, simulamos o RLQ com as restrições de $\mathbf{P}_{\mathbf{v}}^{\mathcal{H}}$ e $\check{\mathbf{P}}_{\mathbf{v}}^{\mathcal{H}}$ e com as realizações $w_{2}$, considerando todas as combinações dos elementos destes 4 conjuntos. Para o cenário 1 , o menor custo foi obtido para a combinação $1.50 \times\left[Q_{i}(t)\right]_{11}, 1.50 \times\left[Q_{i}(t)\right]_{22}$, $0.50 \times\left[R_{i}(t)\right]_{11}$ e $1.33 \times\left[R_{i}(t)\right]_{22}$, que promoveu redução de $0.3 \%$ em relação ao custo obtido com pesos dispostos na Tabela 3.2. Já no cenário 2, obtivemos melhora de $0.70 \%$ com o uso dos pesos $0.83 \times\left[Q_{i}(t)\right]_{11}, 0.66 \times\left[Q_{i}(t)\right]_{22}, 0.50 \times\left[R_{i}(t)\right]_{11}$ e $1.50 \times\left[R_{i}(t)\right]_{22}$, em relação aos pesos originais. Esta análise foi realizada apenas para motivar melhorias no método de obtenção do pesos. Cabe destacar que os resultados apresentados na seção seguinte foram obtidos considerando os valores da tabela acima.

Para aproximar a solução do RLQ com CHF para o RLQ com CMLP, utilizamos $T=1000$ no Teorema 2.2.1. Para ilustrar, apresentamos na Figura 3.4, em azul, o custo do RLQ versus horizonte $T$, para o cenário 1 . As demais curvas se referem aos valores obtidos para os termos que compõem a expressão do custo. Observe o sinal negativo de $v_{\theta(0)}^{0}$ e, principalmente, que o valor do CMLP converge para o termo $\left[w_{\theta(0)}^{k}\right] /(T+1)$, o que condiz com o resultado dado pelo Teorema 2.4.6. Podemos utilizar este teorema, uma vez que o conjunto $\left(\Psi_{R L Q}, \mathcal{W}_{0}^{T}, \mathbb{P}_{v}\right)$ é detetável e o custo para o regulador linear quadrático associado é uniformemente limitado. De fato, como a matriz $Q$ é definida positiva (Tabela 3.2), podemos afirmar que este conjunto é detetável e, até mesmo, observável. Por outro lado, como podemos observar na Seção B.1, a matriz $B(t)$ não é singular, de modo que o regulador quadrático com CMLP associado tem custo uniformemente limitado.

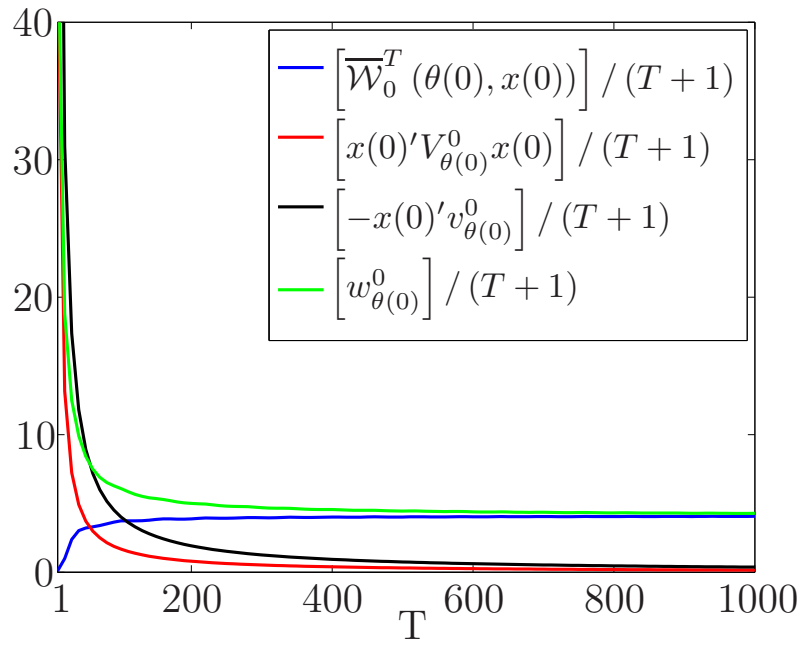

Figura 3.4: Convergência de $\mathcal{W}^{T}$ versus $T$ para o cenário 1.

Por fim, é importante apresentar outra importante simplificação necessária à aplicação do RLQ com SLSM no planejamento hidrotérmico. Como apresentamos no capítulo 
anterior, a variável $u(t)$ depende do valor observado para $\theta(t)$. Entretanto, no problema em questão, não é possível utilizar este valor como entrada para o controle uma vez que as vazões afluentes do estágio $t$ poderão ser mensuradas somente no início do intervalo $t+1$. Assim, o controle de fato implementado foi dado por $u(t)=E\left\{u^{*}(t) \mid \theta(t-1)\right\}$, em que $u^{*}(t)$ é fornecido pelo Teorema 2.2.1.

\subsection{Resultados e Discussão}

Iniciamos apresentando um resultado preliminar que visa avaliar o número de realizações empregado nas simulações e na obtenção das matrizes de peso. Na Figura 3.5, apresentamos o custo esperado e a covariância obtidos pelo RLQ em relação à PDD para o cenário 1. Estes valores estão apresentados de acordo $S$, definido como o número de realizações obtido com a simulação de Monte Carlo. Observe que o valor $S_{2}=1000$ utilizado para obter e investigar os pesos, oferece uma boa estimativa para o custo relativo esperado. Já o número de realizações adotado para as comparações dos controladores, $S_{1}=3000$, garante boa estimativa para a avaliação do custo esperado e também da covariância. A figura condizente ao cenário 2 apresentou convergência similar e está apresentada na Seção B.4 no Apêndice.

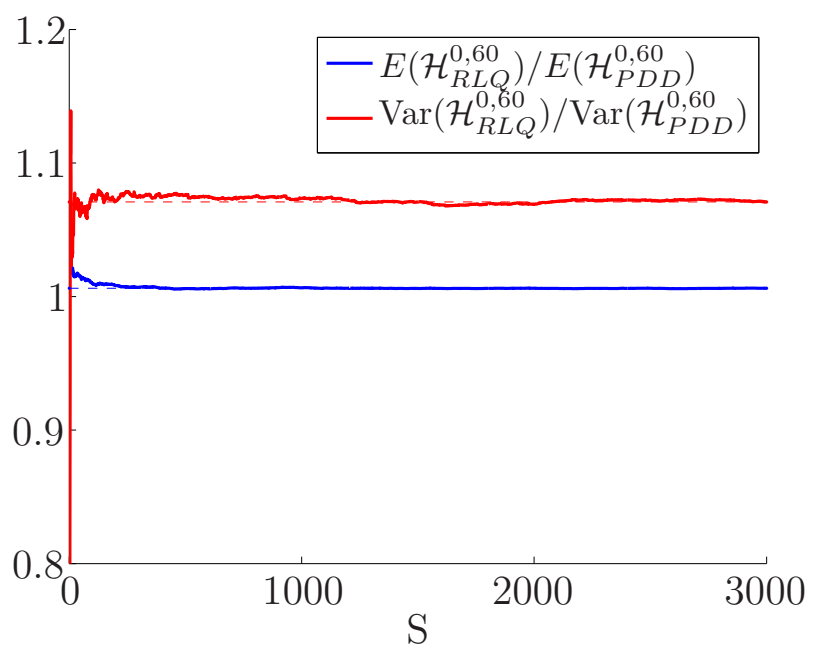

Figura 3.5: Convergência do valor esperado e covariância do custo para o cenário 1.

Os principais resultados gerados nesta aplicação estão dispostos na Tabela 3.3, em que $\bar{s}_{a}\left(w_{1}^{j}\right)$ é definido como o vertimento total de todos os reservatórios durante todo o horizonte de tempo para a realização $w_{1}^{j}$ e controlador $a$. Observe que os dois primeiros valores apresentados pela terceira coluna definem as linhas tracejadas da Figura 3.5. 
Tabela 3.3: Principais resultados obtidos via simulação de Monte Carlo.

\begin{tabular}{rcccc} 
& \multicolumn{2}{c}{ Cenário 1} & \multicolumn{2}{c}{ Cenário 2} \\
& $a=\mathrm{PDE}$ & $a=\mathrm{PDD}$ & $a=\mathrm{PDE}$ & $a=\mathrm{PDD}$ \\
$E\left(\mathcal{H}_{R L Q}^{0,60}\right) / E\left(\mathcal{H}_{a}^{0,60}\right)$ & 1.0263 & 1.0074 & 1.0234 & 0.9989 \\
$\operatorname{Var}\left(\mathcal{H}_{R L Q}^{0,60}\right) / \operatorname{Var}\left(\mathcal{H}_{a}^{0,60}\right)$ & 1.1743 & 1.0709 & 0.9805 & 0.8996 \\
$\mathrm{E}\left\{\bar{s}_{R L Q}\right\} / \mathrm{E}\left\{\bar{s}_{a}\right\}$ & 0.9117 & 0.8826 & 1.0556 & 1.0139 \\
$\min _{j}\left[\mathcal{H}_{R L Q}^{0,60}\left(w_{1}^{j}\right) / \mathcal{H}_{a}^{0,60}\left(w_{1}^{j}\right)\right]-1$ & -0.1938 & -0.3202 & -0.0222 & -0.1446 \\
$\max _{j}\left[\mathcal{H}_{R L Q}^{0,60}\left(w_{1}^{j}\right) / \mathcal{H}_{a}^{0,60}\left(w_{1}^{j}\right)\right]-1$ & 0.1087 & 0.1181 & 0.1134 & 0.1652
\end{tabular}

A partir desta tabela, podemos observar que no cenário 1, o RLQ obteve resultados piores que os controladores obtidos via programação dinâmica com relação ao custo esperado e covariância. Por outro lado, no cenário 2, o RLQ apresentou um custo similar à PDD e uma variância aproximadamente $10 \%$ menor. Variâncias menores são mais interessantes pois significam maior probabilidade de que o custo obtido em cada realização se aproxime do custo esperado. Com relação à PDE, o RLQ obteve resultado melhor para a covariância, porém um custo esperado $2.34 \%$ superior. As melhores e piores realizações do RLQ em termos de custo estão apresentadas pelas duas últimas linhas da tabela. Note que os menores custos do RLQ para o cenário 1, (penúltima linha), são muito menores que os menores custos das programações dinâmicas (última linha). Já para o cenário 2, estes valores se apresentam mais equilibrados principalmente com relação à PDD. Considerando o vertimento, o RLQ apresentou valores relativos maiores no cenário 2 e consideravelmente menores no cenário 1 . O vertimento é indesejável pois aumenta o nível do canal de fuga e diminui a queda bruta do reservatório, prejudicando a potência gerada, como explícito na equação (3.6). Entretanto, como podemos observar pela Tabela 3.3, os valores obtidos para o vertimento não explicam os custos obtidos em ambos os cenários.

Em resumo, considerando principalmente o valor esperado e a covariância do custo hidrotérmico, podemos observar que o RLQ obteve seus melhores resultados no cenário 2. É importante relatar que o volume esperado de água que permanece armazenado nos reservatórios de Sobradinho e Itaparica no final do horizonte também concordou com esta afirmação. No cenário 1, o RLQ obteve um acúmulo de $3.4902 \times 10^{4} \mathrm{hm}^{3}$ de água nestas usinas, o que representa $0.93 \%$ superior ao observado para a PDD e $3.44 \%$ inferior ao obtido para a PDE. Por outro lado, no cenário 2, observamos aproximadamente $3.8341 \times 10^{4} \mathrm{hm}^{3}$ armazenados pelo RLQ nestes reservatórios, o que 
representa $6.22 \%$ a mais que o observado para a PDD e $1.84 \%$ superior ao valor obtido com o uso da PDE.

Na Tabela 3.4 apresentamos a relação entre as variâncias das turbinagens e dos volumes armazenados para os reservatórios de Sobradinho e Itaparica. Variâncias menores para estas variáveis são interessantes pois estão intimamente relacionadas com a confiabilidade do controlador, por exemplo, refletem menor probabilidade dos reservatórios se manterem vazios. No cenário 1, com excessão da variância para a turbinagem de Itaparica, o RLQ obteve valores similares à PDE e, consideravelmente, melhores que a PDD. Já no cenário 2, o RLQ obteve, em geral, valores sensivelmente melhores que a PDE e muito menores que os obtidos via PDD. Somado à estes resultados, é valido também relatar que, no cenário 1, o RLQ utilizou a restrição de volume mínimo de Sobradinho e Itaparica, respectivamente, $11.86 \%$ e $45.11 \%$ a mais que a solução obtida via $\mathrm{PDD}$. Em relação à PDE, os valores foram 26.45\% e 108.54\% superiores, na mesma ordem. Já no cenário 2, os valores obtidos são favoráveis ao RLQ, pois este controlador utilizou as restrições de volume mínimo para Sobradinho e Itaparica, respectivamente, $33.99 \%$ e $48.33 \%$ menos que a PDD e $22.33 \%$ e $9.99 \%$ menos que a PDE.

Tabela 3.4: Relação entre as variâncias do estado e controle.

\begin{tabular}{lcccc} 
& \multicolumn{2}{c}{ Cenário 1} & \multicolumn{2}{c}{ Cenário 2} \\
& $a=\mathrm{PDE}$ & $a=\mathrm{PDD}$ & $a=\mathrm{PDE}$ & $a=\mathrm{PDD}$ \\
$\operatorname{Var}\left(\left[x_{R L Q}(t)\right]_{1}\right) / \operatorname{Var}\left(\left[x_{a}(t)\right]_{1}\right)$ & 1.0137 & 0.8016 & 0.9055 & 0.7290 \\
$\operatorname{Var}\left(\left[x_{R L Q}(t)\right]_{2}\right) / \operatorname{Var}\left(\left[x_{a}(t)\right]_{2}\right)$ & 1.0674 & 0.7483 & 0.9342 & 0.5613 \\
$\operatorname{Var}\left(\left[u_{R L Q}(t)\right]_{1}\right) / \operatorname{Var}\left(\left[u_{a}(t)\right]_{1}\right)$ & 0.9906 & 0.9910 & 0.9532 & 0.9281 \\
$\operatorname{Var}\left(\left[u_{R L Q}(t)\right]_{2}\right) / \operatorname{Var}\left(\left[u_{a}(t)\right]_{2}\right)$ & 1.2976 & 1.2750 & 1.0469 & 1.0103
\end{tabular}

Nas figuras 3.6 e 3.7 apresentamos as razões dos custos versus volume de água relativo observado para Sobradinho em cada realização. O volume de água $\bar{v}\left(w_{1}^{j}\right)$ é definido como a relação entre o volume total afluente observado para a realização $w_{1}^{j}$ e o volume histórico médio para um horizonte de 5 anos. Para melhor visualizar o resultado, apresentamos também a média estimada para a relação dos custos, expressa pela linha vermelha. Podemos notar que, no cenário 1, a média de custos do RLQ foi menor que a PDD em realizações cujo volume afluente foi $5 \%$ superior ao volume histórico médio. Por outro lado, para realizações com baixas vazões afluentes o RLQ apresentou um custo sensivelmente superior. No cenário 2 os resultados seguem uma tendência oposta, já que o RLQ apresentou resultados melhores que a PDD para realizações com menor volume afluente e resultados piores quando estes volumes foram acima da média. 
Já para vazões afluentes extremamente altas, podemos observar notável vantagem para o RLQ.

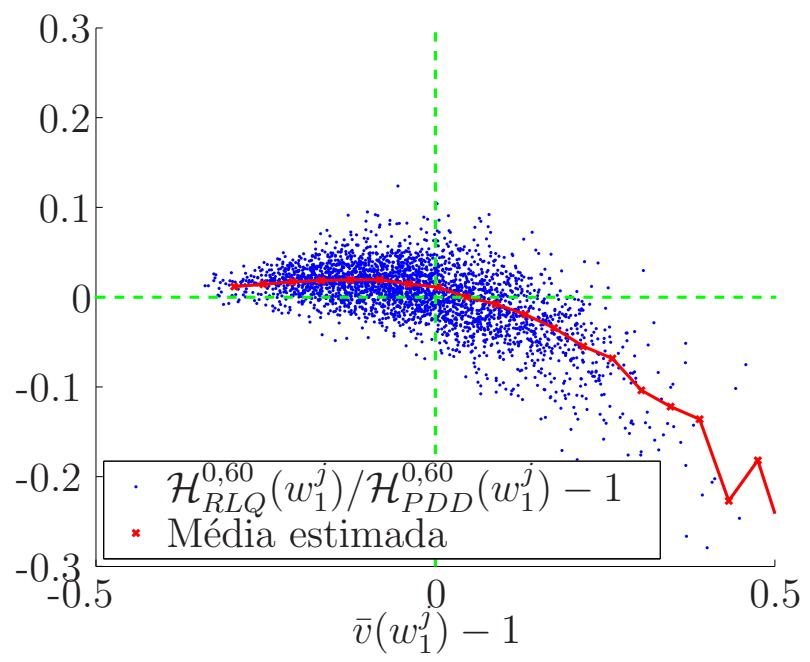

Figura 3.6: Custo hidrotérmico relativo versus volume afluente relativo para o cenário 1.

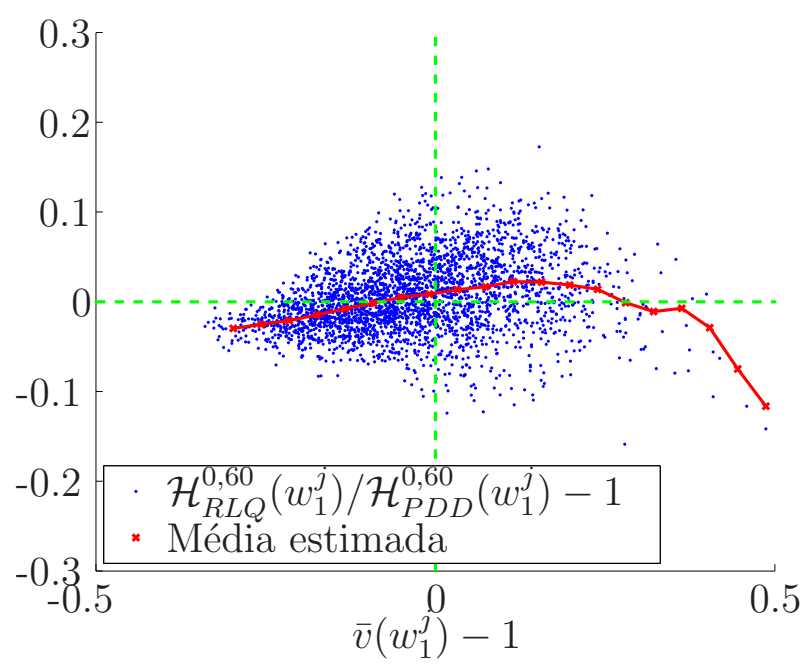

Figura 3.7: Custo hidrotérmico relativo versus volume afluente relativo para o cenário 2.

A diferença entre os resultados de ambos os cenários pode ser compreendida avaliandose o efeito da evaporação no problema. Como já mencionamos, para as usinas estudadas este é um fenômeno importante a ser considerado. De fato, no caso de Sobradinho, a evaporação média estimada é de $132 \mathrm{~m}^{3} / \mathrm{s}$ [25], o que representa uma perda de aproximadamente $5 \%$ de sua vazão afluente média anual. Este efeito pode ser claramente 
observado a partir das diferentes curvas guia obtidas para cada cenário, dispostas nas figuras 3.8 e 3.9. Nestas figuras apresentamos também as trajetórias esperadas para o volume do reservatório de Sobradinho para cada um dos controladores no horizonte de 5 anos. As curvas verdes indicam os limites inferiores e superiores de armazenagem do reservatório e a linha tracejada corresponde à curva guia obtida para o RLQ.

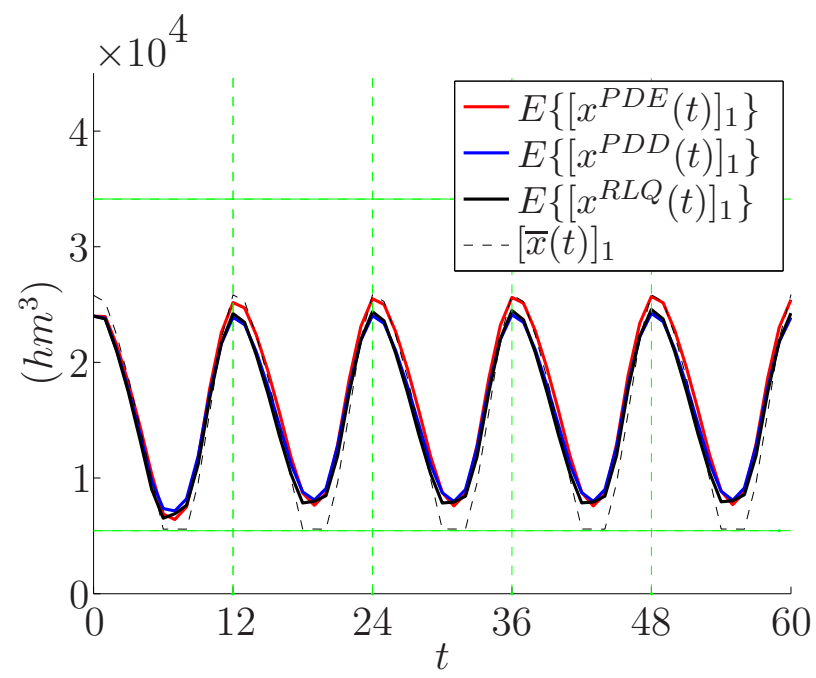

Figura 3.8: Volume esperado para Sobradinho no cenário 1

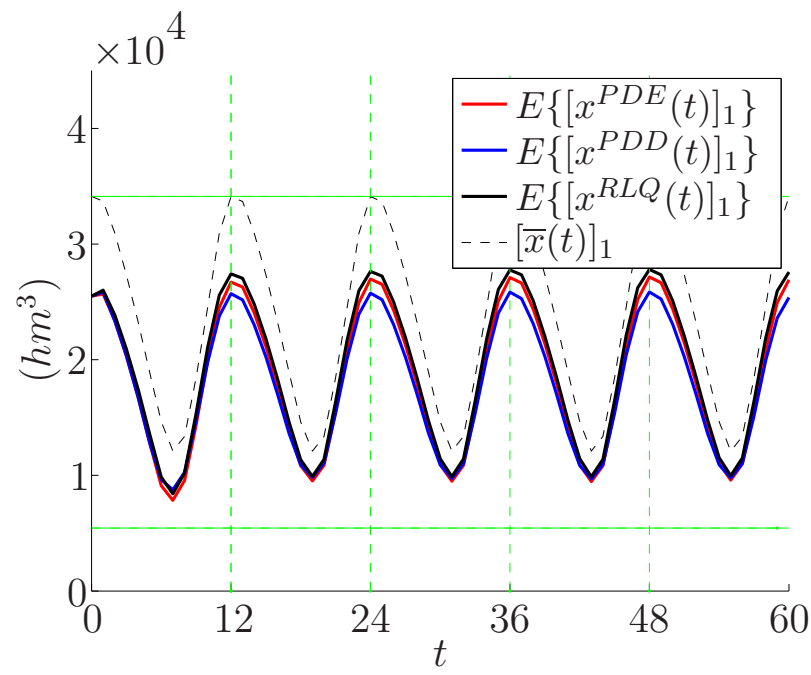

Figura 3.9: Volume esperado para Sobradinho no cenário 2

Níveis mais altos para os reservatórios significam maior área de espelho d'água e, por decorrência, maior perda por evaporação no cenário 1. Em particular, no caso de Sobradinho, a variação da queda bruta com o nível do reservatório é muito pequena, 
como nos mostra a Tabela 3.1, e a área do espelho d'água apresenta uma enorme variação com o nível do reservatório. Estes fatos, somados às altas taxas de evaporação, levam a curva guia para o extremo inferior deste reservatório. Por outro lado, quando o efeito da evaporação é desprezível, a solução para o problema $\check{\mathbf{P}}_{\mathbf{d}}^{\mathcal{H}}$ produz uma trajetória de volume que toca o limite superior do reservatório. Esta estratégia maximiza a queda bruta e, consequentemente, a quantidade de potência gerada, como podemos observar por (3.6). Efeito semelhante, porém menos evidente, pode ser observado para o reservatório de Itaparica, como nos mostram as figuras B.6 e B.7 da Seção B.4 do Apêndice. Nesta seção também estão apresentadas as figuras referentes às trajetórias esperadas para as turbinagens dos reservatórios de Itaparica e Sobradinho.

As curvas guia obtidas para o cenário 1 induzem o RLQ a manter os reservatórios em níveis mais baixos, de modo que este controlador apresentou menor vertimento e menores custos para realizações com alto volume afluente. Por outro lado, para vazões típicas ou baixas, estas curvas guia levam este controlador a utilizar mais vezes as restrições de volume mínimo dos reservatórios, o que explica os piores resultados obtidos pelo RLQ neste cenário. A ativação desta restrição prejudica drasticamente a turbinagem para manter os reservatórios dentro dos limites operacionais, o que provocou maior variância para as turbinagens, principalmente para a usina de Itaparica, como podemos observar pela Tabela 3.4. A turbinagem desta usina tem uma estreita relação com custo, uma vez que sua capacidade de produção é superior à de Sobradinho e sua defluência é ainda o fator preponderante para a definição das potências geradas pelas usinas à fio d'água à jusante, como apresentado pela Figura 3.2. Esta relação com o custo pode também ser observada contrapondo-se os resultados das figuras B.8 e B.9, como também B.10 e B.11.

Note nas figuras 3.8 e 3.9 recém apresentadas que as trajetórias são dependentes da condição inicial em cada cenário. Isto pode ser observado pela mudança do padrão das curvas esperadas para o volume e turbinagem a partir do segundo ano. Lembrando que o RLQ, seguido da PDE, apresentam um maior volume armazenado de água no final do horizonte, podemos esperar que estes controladores apresentam um caráter mais conservador que a PDD no primeiro ano, o que afeta consideravelmente seus desempenhos no horizonte de 5 anos. Para avaliar esta característica, apresentamos nas figuras 3.10 e 3.11 os custos hidrotérmicos para cada ano ao longo do horizonte. Na Figura 3.10 podemos observar que o RLQ mantém o custo ligeiramente maior que a PDD para todos os anos do horizonte. Já na Figura 3.11 é possível notar que o RLQ e PDE apresentaram custos maiores que a PDD no primeiro ano, porém, menores custos nos anos seguintes. 


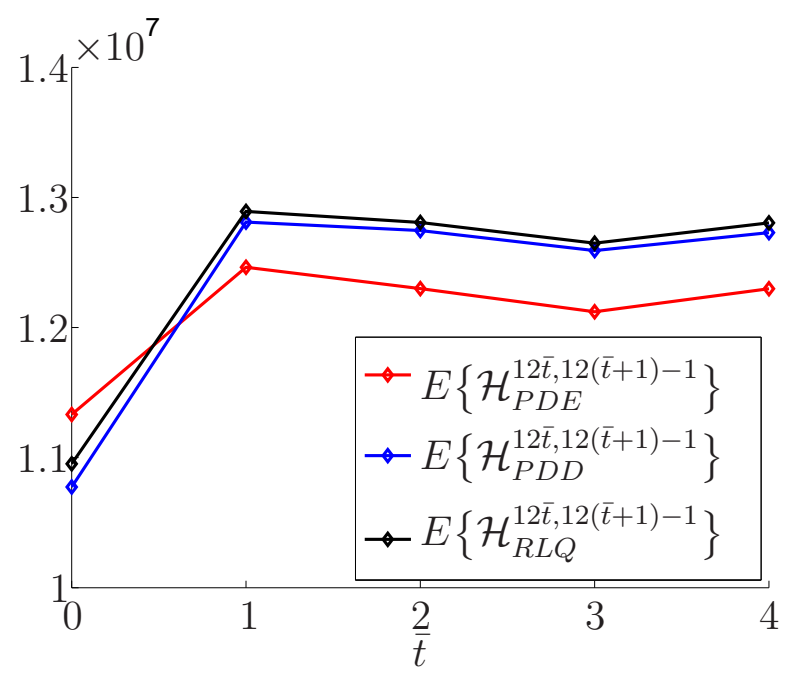

Figura 3.10: Custos esperados em cada ano para o cenário 1.

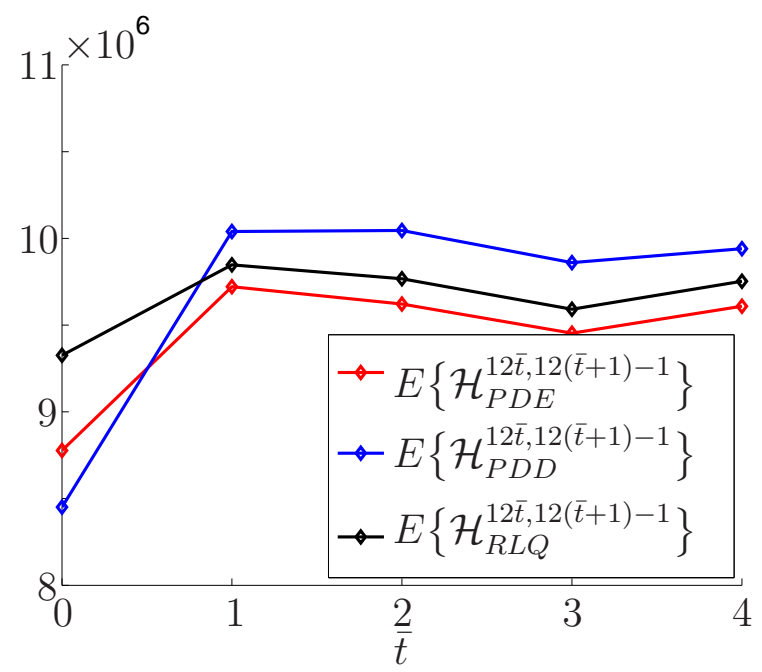

Figura 3.11: Custos esperados em cada ano para o cenário 2.

Diante disto, como mencionamos na Seção 3.2.1, para avaliar o custo dos controladores minimizando o impacto do estado inicial do sistema e o benefício do volume armazenado de água no final do intervalo, simulamos o gerenciamento hidrotérmico utilizando a série histórica da vazão afluente de Sobradinho. Os resultados encontrados estão apresentados na Tabela 3.5. De modo geral, o desempenho do RLQ para o cenário 1 foi similar ao obtido via simulação com séries sintéticas. Vale ressaltar que neste cenário o custo do RLQ foi menor que o da PDD e que a variância destes controladores se apresentou muito maior que a obtida através da PDE. Já no cenário 
2, o custo esperado e variância do RLQ foram muito menores que os valores observados para a PDD. Com relação à PDE, os valores de custo esperado e a variância do RLQ foram ligeiramente maiores. Já os valores relativos à ativação da restrição de volume mínimo foram similares aos encontrados com o uso das séries sintéticas. No cenário 1, o RLQ utilizou esta restrição em Sobradinho $13.84 \%$ a mais que a PDD e a restrição de Itaparica foi utilizada $45.71 \%$ a mais que este mesmo controlador. Em relação à PDE, os valores obtidos foram, na mesma ordem, $27.46 \%$ e $142.86 \%$ maiores. Já para o cenário 2, os valores obtidos são favoráveis ao RLQ, pois, este controlador utilizou as restrições de volume mínimo para Sobradinho e Itaparica, respectivamente, $42.86 \%$ e $57.45 \%$ menos que a PDD, e $29.67 \%$ e $13.04 \%$ menos que a PDE.

Tabela 3.5: Resultados obtidos para a série de vazão real de Sobradinho.

\begin{tabular}{rcccc} 
& \multicolumn{2}{c}{ Cenário 1} & \multicolumn{2}{c}{ Cenário 2} \\
& $a=$ PDE & $a=$ PDD & $a=$ PDE & $a=$ PDD \\
$E\left\{\mathcal{H}_{R L Q}^{0,924}\right\} / E\left\{\mathcal{H}_{a}^{0,924}\right\}$ & 1.0396 & 0.9962 & 1.0071 & 0.9616 \\
$\operatorname{Var}\left(\mathcal{H}_{R L Q}^{0,924}\right) / \operatorname{Var}\left(\mathcal{H}_{a}^{0,924}\right)$ & 1.7219 & 1.1171 & 1.0431 & 0.6818 \\
$\mathrm{E}\left\{\bar{s}_{R L Q}\right\} / \mathrm{E}\left\{\bar{s}_{a}\right\}$ & 0.9004 & 0.8865 & 1.0239 & 1.0002
\end{tabular}

Os resultados obtidos para o cenário 1 sugerem cautela ao encontrar as curvas guia para reservatórios submetidos a altas taxas de evaporação. Nestes casos, acreditamos que a elevação da curva guia para o volume, principalmente para Sobradinho, deve minimizar o uso das restrições de volume mínimo pelo RLQ e, consequentemente, diminuir o custo esperado para este controlador no cenário 1. Isto pode ser alcançado, por exemplo, aumentando as restrições de volume mínimo na definição do problema $\mathbf{P}_{\mathbf{d}}^{\mathcal{H}}$. Porém, esta modificação deve ser feita com cuidado, uma vez que elevar a curva guia para o volume também favorece a perda de água por evaporação, o que, por sua vez, prejudica o desempenho do sistema.

Por outro lado, o desempenho obtido no cenário 2, sugere que o RLQ pode representar uma alternativa interessante para tratar do problema de planejamento da operação de um sistema hidrotérmico. Vale ressaltar que a possibilidade de obtenção de pesos que produzam menores custos hidrotérmicos, como discutido da Seção 3.2.3, também aponta na mesma direção. Os resultados deste cenário mostraram que uma solução previamente obtida pela PDD pode ser beneficiada, com baixo custo computacional, com o uso do RLQ, e que este controlador, além de apresentar custos esperados menores, pode trazer benefícios secundários como menor covariância para o custo e para as variáveis de estado e controle, além de melhores resultados em realizações com 
baixa vazão afluente. Estes resultados também indicam que o desconhecimento da restrição de limite superior para o volume dos reservatórios pouco prejudicou a solução do RLQ, já que este controlador apresentou resultados de vertimento semelhantes em relação à PDD, mesmo mantendo os reservatórios em níveis mais elevados. Além disto, acreditamos que o bom desempenho do RLQ neste cenário mostrou que o conhecimento do modelo de vazões por parte deste controlador compensou suas desvantagens em relação às simplificações assumidas, tais como, aproximação de soluções infactíveis, atendimento a outra função de custo e desconhecimento das restrições do problema. Esta observação corrobora com a afirmação de [19] sobre a vantagem obtida pelo RLQ devido à possibilidade de considerar modelos mais complexos para as vazões.

Apesar deste trabalho ter considerado o desempenho dos controladores sob a ótica da geração energética, é importante ressaltar que o RLQ pode seguir curvas guia que refletem múltiplas preocupações quanto ao uso da água, tais como, recreação, aquicultura, irrigação e abastecimento urbano, e quanto às demais funções de um reservatório, como fornecer proteção contra cheias e manter a qualidade da água. Neste caso, o RLQ deve manter os níveis de volume e turbinagem próximo aos valores pré-estabelecidos enquanto mantém baixo os custos hidrotérmicos pela escolha adequada das matrizes de ponderação. Para isto, acreditamos que o método proposto para encontrar os pesos, com base na solução da PDD, pode ser utilizado sem modificações. 



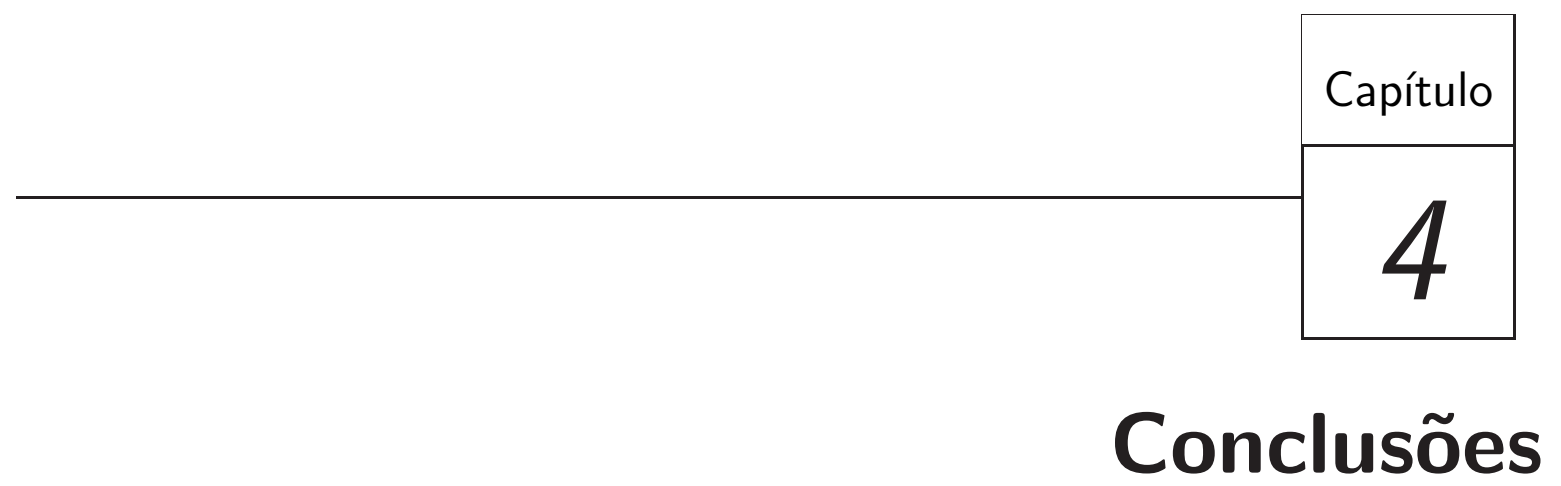

Com relação aos estudos teóricos, nosso principal objetivo foi apresentar condições suficientes para a existência do CMLP associado ao RLQ. Considerando os resultados apresentados no capítulo 2, concluímos que o conceito de detetabilidade uniforme juntamente com a hipótese de que o regulador linear quadrático associado ao problema tenha custo uniformemente limitado, são suficientes para a existência do CMLP e para que o controle obtido seja estabilizante. Estes resultados podem ser compreendidos como generalizações dos estudos de [10] e [26] desde que consideramos os parâmetros variantes no tempo e a cadeia de Markov com dimensão finita, não necessariamente ergódica, e também variante no tempo.

No aspecto prático, utilizamos o sistema de usinas do rio São Francisco em dois casos de estudo para avaliar o desempenho do RLQ em relação à solução ótima, obtida por PDE, e em relação à solução obtida via PDD. A partir dos resultados obtidos para o cenário 2, concluímos que o RLQ pode representar uma alternativa interessante para tratar do problema de planejamento da operação de um sistema hidrotérmico, pois, além de apresentar um custo esperado, covariância do custo e custos em cenário de seca menores que a PDD, este controlador pode trazer ainda benefícios secundários, mesmo em relação à PDE, tais como, menor número de vezes que os reservatórios se mantêm vazios e menor covariância para as variáveis de estado e controle. Além disto, vale mencionar que o método apresentado tem demanda computacional menor que a 
PDE, pois, combina a solução da PDD, que é mais leve que a PDE, com a solução analítica do RLQ. Por outro lado, é importante lembrar que os resultados obtidos para o cenário 1 sugerem cautela ao encontrar as curvas guia para cenários com altas taxas de evaporação. Acreditamos que para diminuir os custos hidrotérmicos do RLQ para este cenário é preciso elevar as curvas guias para o volume, procurando minimizar o uso das restrições de volume mínimo dos reservatórios. Entretanto, esta elevação deve ser feita cuidadosamente, pois forçar as trajetórias de volume a permanecerem em níveis mais elevados também favorece a perda de água por evaporação, o que, por sua vez, prejudica o desempenho do sistema.

Para estender os estudos teóricos, acreditamos que é interessante considerar a cadeia de Markov com dimensão infinita. Com relação à aplicação, trabalhos futuros consistem em avaliar o desempenho do RLQ em outros casos de estudo. Além disto, é importante utilizar outras técnicas para obtenção das curvas guia e matrizes de pesos que não sofram do "mal da dimensionalidade", tais como a programação não-linear e a aproximação empregada em [19], para a obtenção das matrizes de ponderação. 


\section{Referências Bibliográficas}

[1] B. D. O. Anderson and J. B. Moore. Detectability and stabilizability of timevarying discrete-time linear systems. SIAM Journal on Control and Optimization, 19(1):20-32, 1981.

[2] T. W. Archibald, C. S. Buchanan, K. I. M. McKinnon, and L. C. Thomas. Nested Benders decomposition and dynamic programming for reservoir optimisation. Journal of the Operational Research Society, 50(5):468-479, 1999.

[3] T.W. Archibald, K.I.M. McKinnon, and L.C. Thomas. An aggregate stochastic dynamic programming model of multireservoir systems. Water Resources Research, 33(2):333-340, 1997.

[4] M. T. L. Barros, F. T-C. Tsai, S. Yang, J. E. G. Lopes, and W. W-G. Yeh. Optimization of large-scale hydropower system operations. Journal of Water Resources Planning and Management, 129(3):178-188, 2003.

[5] J. L. B. Brandao. Performance of the Equivalent Reservoir Modelling Technique for Multi-Reservoir Hydropower Systems. Water Resources Management, 24(12):3101-3114, SEP 2010.

[6] A. Castelletti, F. Pianosi, and R. Soncini-Sessa. Water reservoir control under economic, social and environmental constraints. Automatica, 44(6):1595 - 1607, 2008. Stochastic Modelling, Control, and Robust Optimization at the Crossroads of Engineering, Environmental Economics, and Finance.

[7] E. F. Costa and J. B. R. do Val. On the detectability and observability of discretetime Markov jump linear systems. System and Control Letters, 44:135-145, 2001. 
[8] E. F. Costa and J. B. R. do Val. Optimal Cost Convergence with Respect to the Time Horizon. In in Proc. Eur. Control Conf. (ECC'03), Cambridge, U.K., 2003.

[9] E.F. Costa and J.B.R. do Val. Uniform approximation of infinite horizon control problems for nonlinear systems and stability of the approximating controls. Automatic Control, IEEE Transactions on, 54(4):881 -886, 2009.

[10] O. L. V. Costa, M. D. Fragoso, and R. P. Marques. Discrete-Time Markovian Jump Linear Systems. Springer-Verlag, 2005.

[11] Oswaldo L.V. Costa and Wanderlei L. de Paulo. Indefinite quadratic with linear costs optimal control of Markov jump with multiplicative noise systems. Automatica, 43(4):587 - 597, 2007.

[12] Operador Nacional do Sistema Elétrico (ONS). Inventário das Restrições Operativas Hidráulicas dos Aproveitamentos Hidrelétricos. Rio de Janeiro RJ, 2009.

[13] Operador Nacional do Sistema Elétrico (ONS). Diretrizes para as Regras de Operação de Controle de Cheias - Bacia do rio São Francisco (Ciclo 2009-2010). Rio de Janeiro RJ, 2010.

[14] J. B. R. do Val and T. Basar. Receding horizon control of jump linear systems and a macroeconomic policy problem. Journal of Economic Dynamics and Control, 23:1099-1131, 1999.

[15] A. Doucet, N. J. Gordon, and V. Krishnamurthy. Particle filters for state estimation of jump Markov linear systems. IEEE Transactions on Signal Processing, 49(3):613-624, 2001.

[16] M. Karamouz and M.H. Houck. Comparison of Stochastic and Deterministic Dynamic-Programming for Reservoir Operating Rule Generation. Water Resources Bulletin, 23(1):1-9, 1987.

[17] M. Karamouz and H. V. Vasiliadis. Bayesian stochastic optimization of reservoir operation using uncertain forecasts. Water Resour. Res., 28(5):1221-1232, 1992.

[18] J. W. Labadie. Optimal operation of multireservoir systems: State-of-the-art review. Journal of Water Resources Planning and Management, 130(2):93-111, 2004.

[19] D. McLaughlin and H. L. Velasco. Real-time control of a system of large hydropower reservoirs. Water Resour. Res., 26(4):623-635, 1990. 
[20] K. D. W. Nandalal and J. J. Bogardi. Dynamic programming based operation of reservoirs: applicability and limits. Cambridge University Press, 2007.

[21] Operador Nacional do Sistema Elétrico. Atualização De Séries Históricas De Vazões - Período 1931 a 200\%. (ONS), Rio de Janeiro RJ, Novembro 2008.

[22] T.B.M.J. Ouarda and J.W. Labadie. Chance-constrained optimal control for multireservoir system optimization and risk analysis. Stochastic Environmental Research and Risk Assessment, 15(3):185-204, 2001.

[23] E.C. Özelkan, Á. Galambosi, E. Fernández-Gaucherand, and L. Duckstein. Linear quadratic dynamic programming for water reservoir management. Applied Mathematical Modelling, 21(9):591 - 598, 1997.

[24] M.V.F. Pereira. Optimal Scheduling of Hydrothermal Systems - An Overview. IFAC - Symposium on Planning and Operation of Electric Energy System, pages $1-9,1985$.

[25] S. B. Pereira, F. F. Pruski, D. D. da Silva, and M. M. Ramos. Evaporação líquida no lago de sobradinho e impactos no escoamento devido à construção do reservatório. Revista Brasileira de Engenharia Agrícola e Ambiental, 13(3):346$352,2009$.

[26] K. D. Running and N. C. Martins. Optimal preview control of markovian jump linear systems. IEEE Transactions on Automatic Control, 54(9):2260-2266, 2009.

[27] G. N. Saridis. Intelligent robotic control. IEEE Transactionson Automatic Control, 28:547-557, 1983.

[28] O.T. Sigvaldason. Simulation-Model for Operating a Multipurpose MultiReservoir System. Water Resources Research, 12(2):263-278, 1976.

[29] S. Soares. Planejamento da Operação de Sistemas Hidrotérmicos. SBA: Controle de Automação, 1(2):122-131, 1987.

[30] S. Soares and A.A.F.M. Carneiro. Optimal Operation of Reservoirs for Electric Generation. Power Delivery, IEEE Transactions on, 6(3):1101 -1107, 1991.

[31] D. D. Sworder and R. O. Rogers. An LQ-solution to a control problem associated with a solar thermal central receiver. IEEE Transactions on Automatic Control, 28(10):971-978, 1983. 
[32] V Ugrinovskii and HR Pota. Decentralized control of power systems via robust control of uncertain Markov jump parameter systems. International Journal of Control, 78(9):662-677, 2005.

[33] D. Wang and B.J. Adams. Optimization of Real-Time Reservoir Operations with Markov Decision-Processes. Water Resources Research, 22(3):345-352, 1986.

[34] S. A. Wasimi and P. K. Kitanidis. Real-time forecasting and daily operation of a multireservoir system during floods by linear quadratic gaussian control. Water Resour. Res., 19(6):1511-1522, 1983.

[35] R. Wurbs. Modeling and Analysis of Reservoir System Operations. Prentice-Hall, 1996

[36] S. Yakowitz. Dynamic-Programming Applications in Water-Resources. Water Resources Research, 18(4):673-696, 1982.

[37] W. W. G. Yeh. Reservoir Management and Operations Models: A State-of-the-Art Review. Water Resources Research, 21(12):1797-1818, 1985. 


\section{A.1 Prova para o RLQ com CHF para SLSM}

Apresentamos abaixo uma prova alternativa para o controlador que soluciona o RLQ com CHF sujeito a um SLSM. Este teorema foi adaptado de [14] para incluir a variação temporal nos parâmetros.

Teorema A.1.1. A solução ótima para o problema intermediário associado ao sistema $\Psi$ pode ser dada por $u_{k}^{* T-1}=\left(u^{*}(k), \ldots, u^{*}(T-1)\right)$, em que $u^{*}(t)=r_{i}^{t}+K_{i}^{t} x$, para $0 \leq k \leq t \leq T-1$, sempre que $i=\theta(t)$ e $x=x(t)$, cujos parâmetros $r_{i}^{t}$ e $K_{i}^{t}$ são dados por

$$
\begin{gathered}
r_{i}^{t}=-\left(\Lambda_{i}^{t+1}\right)^{-1}\left[B_{i}(t)^{\prime}\left(\mathcal{E}_{i}^{t}\left(V^{t+1}\right) e_{i}(t)+\frac{1}{2} \mathcal{E}_{i}^{t}\left(v^{t+1}\right)\right)-R_{i}(t) \bar{u}_{i}(t)\right] e \\
K_{i}^{t}=-\left(\Lambda_{i}^{t+1}\right)^{-1} B_{i}(t)^{\prime} \mathcal{E}_{i}^{t}\left(V^{t+1}\right) A_{i}(t),
\end{gathered}
$$

com

$$
\Lambda_{i}^{t+1}=R_{i}(t)+B_{i}(t)^{\prime} \mathcal{E}_{i}^{t}\left(V^{t+1}\right) B_{i}(t) .
$$


O custo de continuação incorrido por esta sequência de controle pode ser expresso pela função valor que segue,

$$
\overline{\mathcal{W}}_{k}^{T}(i, x)=x^{\prime} V_{i}^{k} x+x^{\prime} v_{i}^{k}+w_{i}^{k}
$$

com funções $V_{i}^{t}, v_{i}^{t}$ e $w_{i}^{t}$ atendendo à seguinte formação

$$
\begin{aligned}
V_{i}^{t}=Q_{i}(t) & +\left(K_{i}^{t}\right)^{\prime} R_{i}(t) K_{i}^{t}+\left(A_{i}(t)+B_{i}(t) K_{i}^{t}\right)^{\prime} \mathcal{E}_{i}^{t}\left(V^{t+1}\right)\left(A_{i}(t)+B_{i}(t) K_{i}^{t}\right), \\
v_{i}^{t}= & \left(A_{i}(t)+B_{i}(t) K_{i}^{t}\right)^{\prime} \mathcal{E}_{i}^{t}\left(v^{t}\right)+2\left(A_{i}(t)+B_{i}(t) K_{i}^{t}\right)^{\prime} \mathcal{E}_{i}^{t}\left(V^{t}\right) e_{i}(t) \\
& -2\left(K_{i}^{t}\right)^{\prime} R(t) \bar{u}_{i}(t)-2 Q_{i}(t) \bar{x}_{i}(t), \quad e \\
w_{i}^{t}= & \mathcal{E}_{i}^{t}\left(w^{t+1}\right)+\bar{x}_{i}(t)^{\prime} Q_{i}(t) \bar{x}_{i}(t)+\bar{u}_{i}^{\prime}(t) R_{i}(t) \bar{u}_{i}(t)+e_{i}^{\prime}(t) \mathcal{E}_{i}^{t}\left(V^{t+1}\right) e_{i}(t) \\
+ & \mathcal{E}_{i}^{t}\left(v^{t+1}\right)^{\prime} e_{i}(t)-\left(r_{i}^{t}\right)^{\prime} \Lambda_{i}^{t+1} r_{i}^{t}+\operatorname{tr}\left[H_{i}(t) H_{i}(t)^{\prime} \mathcal{E}_{i}^{t}\left(V^{t+1}\right)\right]
\end{aligned}
$$

com $V_{i}^{T}=F_{i}(T), v_{i}^{T}=-2 F_{i}(T) \bar{x}_{i}(T)$ e $w_{i}^{T}=\bar{x}_{i}^{\prime}(T) F_{i}(T) \bar{x}_{i}(T)$.

Demonstração: A prova segue por indução em $t$. Para compactar as equações, faremos $\theta(T-1)=i$, e $\bar{t}=T-1$. Para o último instante da sequência podemos utilizar as equações $(2.7)$ e (2.8) para escrever a função valor como

$$
\begin{aligned}
& \overline{\mathcal{W}}_{\bar{t}}^{T}(i, x(\bar{t})) \\
& =\inf _{u_{\bar{t}}^{\bar{t}}}\left\{\mathcal{W}_{\bar{t}}^{T}\left(i, x(\bar{t}), u_{\bar{t}}^{\bar{t}}\right)\right\} \\
& =\inf _{u_{\bar{t}}}\left\{E\left[p(\bar{t})+q(T) \mid \mathfrak{G}_{\bar{t}}\right]\right\} \\
& =\inf _{u_{\bar{t}}}\left\{E \left[\left(x(\bar{t})-\bar{x}_{i}(\bar{t})\right)^{\prime} Q_{i}(\bar{t})\left(x(\bar{t})-\bar{x}_{i}(\bar{t})\right)+\left(u(\bar{t})-\bar{u}_{i}(\bar{t})\right)^{\prime} R_{i}(\bar{t})\left(u(\bar{t})-\bar{u}_{i}(\bar{t})\right)\right.\right. \\
& \left.\left.\quad+\left(x(T)-\bar{x}_{\theta(T)}(T)\right)^{\prime} F_{\theta(T)}\left(x(T)-\bar{x}_{\theta(T)}(T)\right) \mid \mathfrak{G}_{\bar{t}}\right]\right\} \\
& =\inf _{u_{\bar{t}}}\left\{\left(x(\bar{t})-\bar{x}_{i}(\bar{t})\right)^{\prime} Q_{i}(\bar{t})\left(x(\bar{t})-\bar{x}_{i}(\bar{t})\right)+\left(u(\bar{t})-\bar{u}_{i}(\bar{t})\right)^{\prime} R_{i}(\bar{t})\left(u(\bar{t})-\bar{u}_{i}(\bar{t})\right)\right. \\
& \left.\quad+E\left[x(T)^{\prime} F_{\theta(T)} x(T)-2 x(T)^{\prime} F_{\theta(T)} \bar{x}_{\theta(T)}(T)+\bar{x}_{\theta(T)}(T)^{\prime} F_{\theta(T)} \bar{x}_{\theta(T)}(T) \mid \mathfrak{G}_{\bar{t}}\right]\right\}
\end{aligned}
$$

Considerando as definições de $V_{i}^{T}, v_{i}^{T}$ e $w_{i}^{T}$, é ainda verdade que

$$
\begin{aligned}
& \overline{\mathcal{W}}_{\bar{t}}^{T}(i, x(\bar{t}))= \\
& \quad \inf _{u_{\bar{t}}}\left\{\left(x(\bar{t})-\bar{x}_{i}(\bar{t})\right)^{\prime} Q_{i}(\bar{t})\left(x(\bar{t})-\bar{x}_{i}(\bar{t})\right)+\left(u(\bar{t})-\bar{u}_{i}(\bar{t})\right)^{\prime} R_{i}(\bar{t})\left(u(\bar{t})-\bar{u}_{i}(\bar{t})\right)\right. \\
& \left.\quad+E\left[x(T)^{\prime} V_{\theta(T)}^{T} x(T)+x(T)^{\prime} v_{\theta(T)}^{T}+w_{\theta(T)}^{T} \mid \mathfrak{G}_{\bar{t}}\right]\right\} .
\end{aligned}
$$


Utilizando (2.6) podemos escrever

$$
\begin{aligned}
& \overline{\mathcal{W}}_{\bar{t}}^{T}(i, x(\bar{t})) \\
&=\inf _{u_{\bar{t}}}\left\{\left(x(\bar{t})-\bar{x}_{i}(\bar{t})\right)^{\prime} Q_{i}(\bar{t})\left(x(\bar{t})-\bar{x}_{i}(\bar{t})\right)+\left(u(\bar{t})-\bar{u}_{i}(\bar{t})\right)^{\prime} R_{i}(\bar{t})\left(u(\bar{t})-\bar{u}_{i}(\bar{t})\right)\right. \\
&+E\left[\left(A_{i}(\bar{t}) x(\bar{t})+B_{i}(\bar{t}) u(\bar{t})+e_{i}(\bar{t})+H_{i}(\bar{t}) \nu(\bar{t})\right)^{\prime} V_{\theta(T)}^{T}\right. \\
&\left(A_{i}(\bar{t}) x(\bar{t})+B_{i}(\bar{t}) u(\bar{t})+e_{i}(\bar{t})+H_{i}(\bar{t}) \nu(\bar{t})\right) \\
&+\left.\left.\left(A_{i}(\bar{t}) x(\bar{t})+B_{i}(\bar{t}) u(\bar{t})+e_{i}(\bar{t})+H_{i}(\bar{t}) \nu(\bar{t})\right)^{\prime} v_{\theta(T)}^{T}+w_{\theta(T)}^{T} \mid \mathfrak{G}_{\bar{t}}\right]\right\} \\
&=\inf _{u_{\bar{t}}}\{\left(x(\bar{t})-\bar{x}_{i}(\bar{t})\right)^{\prime} Q_{i}(\bar{t})\left(x(\bar{t})-\bar{x}_{i}(\bar{t})\right)+\left(u(\bar{t})-\bar{u}_{i}(\bar{t})\right)^{\prime} R_{i}(\bar{t})\left(u(\bar{t})-\bar{u}_{i}(\bar{t})\right) \\
&+ E\left[\left(A_{i}(\bar{t}) x(\bar{t})+B_{i}(\bar{t}) u(\bar{t})+e_{i}(\bar{t})\right)^{\prime} V_{\theta(T)}^{T}\left(A_{i}(\bar{t}) x(\bar{t})+B_{i}(\bar{t}) u(\bar{t})+e_{i}(\bar{t})\right)\right. \\
&+\left.\left(A_{i}(\bar{t}) x(\bar{t})+B_{i}(t) u(\bar{t})+e_{i}(\bar{t})\right)^{\prime} v_{\theta(T)}^{T}+w_{\theta(T)}^{T} \mid \mathfrak{G}_{\bar{t}}\right] \\
&+ {\left[\left(A_{i}(\bar{t}) x(\bar{t})\right)^{\prime} V_{\theta(T)}^{T} H_{i}(\bar{t}) \nu(\bar{t})+\left(B_{i}(\bar{t}) u(\bar{t})\right)^{\prime} V_{\theta(T)}^{T} H_{i}(\bar{t}) \nu(\bar{t})\right.} \\
&+e_{i}(\bar{t})^{\prime} V_{\theta(T)}^{T} H_{i}(\bar{t}) \nu(\bar{t})+\left(H_{i}(\bar{t}) \nu(\bar{t})\right)^{\prime} V_{\theta(T)}^{T} A_{i}(\bar{t}) x(\bar{t}) \\
&+\left(H_{i}(\bar{t}) \nu(\bar{t})\right)^{\prime} V_{\theta(T)}^{T} B_{i}(\bar{t}) u(\bar{t})+\left(H_{i}(\bar{t}) \nu(\bar{t})\right)^{\prime} V_{\theta(T)}^{T} e_{i}(\bar{t}) \\
&\left.\left.+\left(H_{i}(\bar{t}) \nu(\bar{t})\right)^{\prime} V_{\theta(T)}^{T} H_{i}(\bar{t}) \nu(\bar{t})+\left(H_{i}(\bar{t}) \nu(\bar{t})\right)^{\prime} v_{\theta(T)}^{T} \mid \mathfrak{G}_{\bar{t}}\right]\right\} .
\end{aligned}
$$

Mas sabemos de [10] que para duas funções $\mathfrak{G}_{t}$-mensuráveis $f$ e $g$ independentes de $\nu(t)$ é válido que

$$
E\left[f(\nu(t)) g(\theta(t+1)) \mid \mathfrak{G}_{t}\right]=E\left(f\left(\nu(t) \mid \mathfrak{G}_{t}\right) \mathcal{E}_{\theta(t)}^{t}(g)\right.
$$

Então, utilizando ainda a independência do ruido em relação a $x(\bar{t}), u(\bar{t})$ e $e(\bar{t})$, e a propriedade de invariância do traço em relação a uma permutação cíclica, obtemos

$$
\begin{aligned}
& \overline{\mathcal{W}}_{\bar{t}}^{T}(i, x(\bar{t})) \\
&=\inf _{u_{\bar{t}}}\left\{\left(x(\bar{t})-\bar{x}_{i}(\bar{t})\right)^{\prime} Q_{i}(\bar{t})\left(x(\bar{t})-\bar{x}_{i}(\bar{t})\right)+\left(u(\bar{t})-\bar{u}_{i}(\bar{t})\right)^{\prime} R_{i}(\bar{t})\left(u(\bar{t})-\bar{u}_{i}(\bar{t})\right)\right. \\
&+E\left[\left(A_{i}(\bar{t}) x(\bar{t})+B_{i}(\bar{t}) u(\bar{t})+e_{i}(\bar{t})\right)^{\prime} V_{\theta(T)}^{T}\left(A_{i}(\bar{t}) x(\bar{t})+B_{i}(\bar{t}) u(\bar{t})+e_{i}(\bar{t})\right)\right. \\
&\left.+\left(A_{i}(\bar{t}) x(\bar{t})+B_{i}(\bar{t}) u(\bar{t})+e_{i}(\bar{t})\right)^{\prime} v_{\theta(T)}^{T}+w_{\theta(T)}^{T} \mid \mathfrak{G}_{\bar{t}}\right] \\
&\left.+E\left[\nu(\bar{t})^{\prime} H_{i}(\bar{t})^{\prime} V_{\theta(T)}^{T} H_{i}(\bar{t}) \nu(\bar{t}) \mid \mathfrak{G}_{\bar{t}}\right]\right\},
\end{aligned}
$$


ou ainda,

$$
\begin{aligned}
\overline{\mathcal{W}}_{\bar{t}}^{T}(i, x(\bar{t})) & \\
= & \inf _{u_{\bar{t}}}\left\{\left(x(\bar{t})-\bar{x}_{i}(\bar{t})\right)^{\prime} Q_{i}(\bar{t})\left(x(\bar{t})-\bar{x}_{i}(\bar{t})\right)+\left(u(\bar{t})-\bar{u}_{i}(\bar{t})\right)^{\prime} R_{i}(\bar{t})\left(u(\bar{t})-\bar{u}_{i}(\bar{t})\right)\right. \\
& +\left(A_{i}(\bar{t}) x(\bar{t})+B_{i}(\bar{t}) u(\bar{t})+e_{i}(\bar{t})\right)^{\prime} \mathcal{E}_{i}^{\bar{t}}\left(V^{T}\right)\left(A_{i}(\bar{t}) x(\bar{t})+B_{i}(\bar{t}) u(\bar{t})+e_{i}(\bar{t})\right) \\
& \left.+\left(A_{i}(\bar{t}) x(\bar{t})+B_{i}(\bar{t}) u(\bar{t})+e_{i}(\bar{t})\right)^{\prime} \mathcal{E}_{i}^{\bar{t}}\left(v^{T}\right)+\mathcal{E}_{i}^{\bar{t}}\left(w^{T}\right)+\operatorname{tr}\left[H_{i}(\bar{t}) \Sigma H_{i}(\bar{t})^{\prime} \mathcal{E}_{i}^{\bar{t}}\left(V^{T}\right)\right]\right\} .
\end{aligned}
$$

A equação anterior é quadrática em relação $u(\bar{t})$ de modo que o mínimo pode ser obtido utilizando a condição de derivada primeira igual a zero, o que nos conduz à seguinte condição

$$
\begin{aligned}
u_{i}(\bar{t})=- & \left(R_{i}(\bar{t})+B_{i}(\bar{t})^{\prime} \mathcal{E}_{i}^{\bar{t}}\left(V^{T}\right) B_{i}(\bar{t})\right)^{-1} \\
& {\left[B_{i}(\bar{t})^{\prime}\left(\mathcal{E}_{i}^{\bar{t}}\left(V^{T}\right) e_{i}(\bar{t})+\frac{1}{2} \mathcal{E}_{i}^{\bar{t}}\left(v^{T}\right)-R_{i}(\bar{t}) \bar{u}_{i}\right)+B_{i}(\bar{t})^{\prime} \mathcal{E}_{i}^{\bar{t}}\left(V^{T}\right) A_{i}(\bar{t}) x(\bar{t})\right], }
\end{aligned}
$$

ou ainda, simplesmente, $u_{i}(\bar{t})=r_{i}^{\bar{t}}+K_{i}^{\bar{t}} x(\bar{t})$. Utilizando esta última expressão, podemos desenvolver (A.9) e obter

$$
\begin{gathered}
\overline{\mathcal{W}}_{\bar{t}}^{T}(i, x(\bar{t})) \\
=x(\bar{t})^{\prime}\left[Q_{i}(\bar{t})+\left(K_{i}^{\bar{t}}\right)^{\prime} R_{i}(\bar{t}) K_{i}^{\bar{t}}+A_{i}(\bar{t})^{\prime} \mathcal{E}_{i}^{\bar{t}}\left(V^{T}\right) A_{i}(\bar{t})+\left(K_{i}^{\bar{t}}\right)^{\prime} B_{i}(\bar{t})^{\prime} \mathcal{E}_{i}^{\bar{t}}\left(V^{T}\right) A_{i}(\bar{t})\right. \\
\left.+A_{i}(\bar{t})^{\prime} \mathcal{E}_{i}^{\bar{t}}\left(V^{T}\right) B_{i}(\bar{t}) K_{i}^{\bar{t}}+\left(K_{i}^{\bar{t}}\right)^{\prime} B_{i}(\bar{t})^{\prime} \mathcal{E}_{i}^{\bar{t}}\left(V^{T}\right) B_{i}(\bar{t}) K_{i}^{\bar{t}}\right] x(\bar{t}) \\
+x(\bar{t})^{\prime}\left[-2 Q_{i}(\bar{t}) \bar{x}_{i}(\bar{t})+2\left(K_{i}^{\bar{t}}\right)^{\prime} R_{i}(\bar{t}) r_{i}^{\bar{t}}-2\left(K_{i}^{\bar{t}}\right)^{\prime} R_{i}(\bar{t}) \bar{u}_{i}(\bar{t})\right. \\
+2 A_{i}(\bar{t})^{\prime} \mathcal{E}_{i}^{\bar{t}}\left(V^{T}\right) B_{i}(\bar{t}) r_{i}^{\bar{t}}+2 A_{i}(\bar{t})^{\prime} \mathcal{E}_{i}^{\bar{t}}\left(V^{T}\right) e_{i}(\bar{t}) \\
+2\left(K_{i}^{\bar{t}}\right)^{\prime} B_{i}(\bar{t})^{\prime} \mathcal{E}_{i}^{\bar{t}}\left(V^{T}\right) B_{i}(\bar{t})_{i}^{\bar{t}}+2\left(K_{i}^{\bar{t}}\right)^{\prime} B_{i}(\bar{t})^{\prime} \mathcal{E}_{i}^{\bar{t}}\left(V^{T}\right) e_{i}(\bar{t}) \\
\left.+A_{i}(\bar{t})^{\prime} \mathcal{E}_{i}^{\bar{t}}\left(v^{T}\right)+\left(K_{i}^{\bar{t}}\right)^{\prime} B_{i}(\bar{t})^{\prime} \mathcal{E}_{i}^{\bar{t}}\left(v^{T}\right)\right] \\
+\bar{x}_{i}(\bar{t})^{\prime} Q_{i}(\bar{t}) \bar{x}_{i}(\bar{t})+\left(r_{i}^{\bar{t}}\right)^{\prime} R_{i}(\bar{t}) r_{i}^{\bar{t}}-2\left(r_{i}^{\bar{t}}\right)^{\prime} R_{i}\left(\bar{t} \bar{u}_{i}(\bar{t})+\bar{u}_{i}(\bar{t})^{\prime} R_{i}(\bar{t}) \bar{u}_{i}(\bar{t})\right. \\
+\left(r_{i}^{\bar{t}}\right)^{\prime} B_{i}(\bar{t})^{\prime} \mathcal{E}_{i}^{\bar{t}}\left(V^{T}\right) B_{i}(\bar{t}) r_{i}^{\bar{t}}+2\left(r_{i}^{\bar{t}}\right)^{\prime} B_{i}(\bar{t})^{\prime} \mathcal{E}_{i}^{\bar{t}}\left(V^{T}\right) e_{i}(\bar{t})+e_{i}(\bar{t})^{\prime} \mathcal{E}_{i}^{\bar{t}}\left(V^{T}\right) e_{i}(\bar{t}) \\
+\left(r_{i}^{\bar{t}}\right)^{\prime} B_{i}(\bar{t})^{\prime} \mathcal{E}_{i}^{\bar{t}}\left(v^{T}\right)+e_{i}(\bar{t})^{\prime} \mathcal{E}_{i}^{\bar{t}}\left(v^{T}\right)+\mathcal{E}_{i}^{\bar{t}}\left(w^{T}\right)+\operatorname{tr}\left[H_{i}(\bar{t}) \Sigma H_{i}(\bar{t})^{\prime} \mathcal{E}_{i}^{\bar{t}}\left(V^{T}\right)\right]
\end{gathered}
$$


Observe que os termos dentro do segundo colchete podem ser simplificados colocando-se $\mathcal{E}_{i}^{\bar{t}}\left(v^{T}\right)$ e $\mathcal{E}_{i}^{\bar{t}}\left(V^{T}\right) e_{i}(\bar{t})$ em evidência e considerando as seguinte igualdades

$$
2\left(K_{i}^{\bar{t}}\right)^{\prime} R_{i}(\bar{t}) r_{i}^{\bar{t}}+2\left(K_{i}^{\bar{t}}\right)^{\prime} B_{i}(\bar{t})^{\prime} \mathcal{E}_{i}^{\bar{t}}\left(V^{T}\right) B_{i}(\bar{t}) r_{i}^{\bar{t}}=2\left(K_{i}^{\bar{t}}\right)^{\prime} \Lambda_{i}^{T} r_{i}^{\bar{t}}
$$

e

$$
2 A_{i}(\bar{t})^{\prime} \mathcal{E}_{i}^{t}\left(V^{T}\right) B_{i}(\bar{t}) r_{i}^{\bar{t}}=2 A_{i}(\bar{t})^{\prime} \mathcal{E}_{i}^{t}\left(V^{T}\right) B_{i}(\bar{t})\left(\Lambda_{i}^{T}\right)^{-1} \Lambda_{i}^{T} r_{i}^{\bar{t}}=-2\left(K_{i}^{\bar{t}}\right)^{\prime} \Lambda_{i}^{T} r_{i}^{\bar{t}}
$$

Os termos subsequentes podem também ser reapresentados de forma mais simples, considerando o seguinte

$$
\begin{aligned}
& -2\left(r_{i}^{\bar{t}}\right)^{\prime} R_{i}(\bar{t}) \bar{u}_{i}(\bar{t})+2\left(r_{i}^{\bar{t}}\right)^{\prime} B_{i}(\bar{t})^{\prime} \mathcal{E}_{i}^{\bar{t}}\left(V^{T}\right) e_{i}(\bar{t})+\left(r_{i}^{\bar{t}}\right)^{\prime} B_{i}(\bar{t})^{\prime} \mathcal{E}_{i}^{\bar{t}}\left(v^{T}\right) \\
& =2\left(r_{i}^{\bar{t}}\right)^{\prime} \Lambda_{i}^{T}\left(\Lambda_{i}^{T}\right)^{-1}\left[B_{i}(\bar{t})^{\prime}\left(\mathcal{E}_{i}^{\bar{t}}\left(V^{T}\right) e_{i}(\bar{t})+\frac{1}{2} \mathcal{E}_{i}^{\bar{t}}\left(v^{T}\right)\right)-R_{i}(\bar{t}) \bar{u}_{i}(\bar{t})\right] \\
& =-2\left(r_{i}^{\bar{t}}\right)^{\prime} \Lambda_{i}^{T} r_{i}^{\bar{t}}
\end{aligned}
$$

Logo, lembrando a definição $A_{i}^{t} \triangleq A_{i}(t)+B_{i}(t) K_{i}^{t}$, o custo para $t=\bar{t}$ pode ser dado por

$$
\begin{aligned}
\overline{\mathcal{W}}_{\bar{t}}^{T}(i, x(\bar{t})) \\
=x(\bar{t})^{\prime}\left[Q_{i}(\bar{t})+\left(K_{i}^{\bar{t}}\right)^{\prime} R_{i}(\bar{t}) K_{i}^{\bar{t}}+\left(A_{i}^{\bar{t}}\right)^{\prime} \mathcal{E}_{i}^{\bar{t}}\left(V^{T}\right) A_{i}^{\bar{t}}\right] x(\bar{t}) \\
\quad+x(\bar{t})^{\prime}\left[\left(A_{i}^{\bar{t}}\right)^{\prime} \mathcal{E}_{i}^{\bar{t}}\left(v^{T}\right)+2\left(A_{i}^{\bar{t}}\right)^{\prime} \mathcal{E}_{i}^{\bar{t}}\left(V^{T}\right) e_{i}(\bar{t})-2\left(K_{i}^{\bar{t}}\right)^{\prime} R(\bar{t}) \bar{u}_{i}(\bar{t})-2 Q_{i}(\bar{t}) \bar{x}_{i}(\bar{t})\right] \\
\quad+\mathcal{E}_{i}^{\bar{t}}\left(w^{T}\right)+\bar{x}_{i}(\bar{t})^{\prime} Q_{i}(\bar{t}) \bar{x}_{i}(\bar{t})+\bar{u}_{i}(\bar{t})^{\prime} R_{i}(\bar{t}) \bar{u}_{i}(\bar{t})+e_{i}(\bar{t})^{\prime} \mathcal{E}_{i}^{\bar{t}}\left(V^{T}\right) e_{i}(\bar{t}) \\
\quad+e_{i}(\bar{t})^{\prime} \mathcal{E}_{i}^{\bar{t}}\left(v^{T}\right)-\left(r_{i}^{\bar{t}}\right)^{\prime} \Lambda_{i}^{T} r_{i}^{\bar{t}}+\operatorname{tr}\left[H_{i}(\bar{t}) \Sigma H_{i}(\bar{t})^{\prime} \mathcal{E}_{i}^{\bar{t}}\left(V^{T}\right)\right]
\end{aligned}
$$

ou ainda,

$$
\overline{\mathcal{W}}_{T-1}^{T}(i, x(T-1))=x(T-1)^{\prime} V_{i}^{T-1} x(T-1)+x(T-1)^{\prime} v_{i}^{T-1}+w_{i}^{T-1},
$$

o que está de acordo com a expressão (2.12).

Continuando com a prova, considere que seja válida a seguinte hipótese de indução para $t=k+1$,

$$
\overline{\mathcal{W}}_{k+1}^{T}(\theta(k+1), x(k+1))=x(k+1)^{\prime} V_{\theta(k+1)}^{k+1} x(k+1)+x(k+1)^{\prime} v_{\theta(k+1)}^{k+1}+w_{\theta(k+1)}^{k+1}
$$


Para $t=k$ temos, por definição,

$$
\begin{aligned}
\overline{\mathcal{W}}_{k}^{T}(\theta(k), x(k)) & =\inf _{u_{k}^{T-1}}\left\{E\left[\sum_{t=k}^{T-1} p(t)+q(T) \mid \mathfrak{G}_{k}\right]\right\} \\
& =\inf _{u_{k}}\left\{p(k)+\inf _{u_{k+1}^{T}} E\left[\sum_{t=k+1}^{T-1} p(t)+q(T) \mid \mathfrak{G}_{k}\right]\right\}
\end{aligned}
$$

Utilizando a hipótese de indução, note ainda que são válidas as seguintes igualdades

$$
\begin{aligned}
\overline{\mathcal{W}}_{k}^{T}(\theta(k), x(k))=\inf _{u_{k}}\left\{p(k)+E\left[\inf _{u_{k+1}^{T}} E\left[\sum_{t=k+1}^{T-1} p(t)+q(T) \mid \mathfrak{G}_{k+1}\right] \mid \mathfrak{G}_{k}\right]\right\} \\
=\inf _{u_{k}}\left\{p(k)+E\left[\overline{\mathcal{W}}_{k+1}^{T}(\theta(k+1), x(k+1)) \mid \mathfrak{G}_{k}\right]\right\} \\
=\inf _{u_{k}}\left\{\left(x(k)-\bar{x}_{\theta(k)}(k)\right)^{\prime} Q_{\theta(k)}(k)\left(x(k)-\bar{x}_{\theta(k)}(k)\right)\right. \\
+\left(u(k)-\bar{u}_{\theta(k)}(k)\right)^{\prime} R_{\theta(k)}(k)\left(u(k)-\bar{u}_{\theta(k)}(k)\right) \\
\left.+E\left[x(k+1)^{\prime} V_{\theta(k+1)}^{k+1} x(k+1)+x(k+1)^{\prime} v_{\theta(k+1)}^{k+1}+w_{\theta(k+1)}^{k+1} \mid \mathfrak{G}_{k}\right]\right\}
\end{aligned}
$$

Note que a equação anterior é semelhante à (A.7), assim, aplicando os mesmos procedimentos, é possível obter o controle ótimo dado por $u_{k}^{*}=r_{i}^{k}+K_{i}^{k}$ e a função valor $\overline{\mathcal{W}}_{k}^{T}(i, x(k))=x(k)^{\prime} V_{i}^{k} x(k)+x(k)^{\prime} v_{i}^{k}+w_{i}^{k}$, sempre que $i=\theta(k)$, o que completa a prova. 


\section{A.2 Expressões Alternativas para o CHF}

Proposição A.2.1. Seja uma sequência $L^{t} \in \mathcal{M}^{n 0}$, definida para $0 \leq t \leq T$ e dada por $L^{t}=Q^{t}+\mathcal{L}^{t}\left(L^{t+1}\right)$, com $Q^{t} \triangleq Q(t)+\left(K^{t}\right)^{\prime} R(t) K^{t}$ e $L^{T}=Q^{T}=F(T)$. Então, podemos reapresentar o custo (2.17) como $\breve{\mathcal{W}}_{k}^{T}(i, x)=\sum_{t=k}^{T}\left\langle Q^{t}, \breve{\mathcal{X}}^{t}\right\rangle=\left\langle L^{k}, \breve{\mathcal{X}}^{k}\right\rangle$.

Demonstração: Utilizando a definição (2.17) é válido que

$$
\begin{aligned}
\breve{\mathcal{W}}_{k}^{T}(i, x) & \\
& =E\left(\sum_{t=k}^{T-1} x(t)^{\prime} Q_{\theta(t)}(t) x(t)+u(t)^{\prime} R_{\theta(t)}(t) u(t)+x(T)^{\prime} F_{\theta(T)}(T) x(T)\right) \\
& =E\left(\sum_{t=k}^{T-1} x(t)^{\prime}\left(Q_{\theta(t)}(t)+\left(K^{t}\right)^{\prime} R_{\theta(t)}(t) K^{t}\right) x(t)+x(T)^{\prime} F_{\theta(T)}(T) x(T)\right) \\
& =E\left(\sum_{t=k}^{T} \operatorname{tr}\left[Q_{\theta(t)}^{t} x(t) x(t)^{\prime}\right]\right) \\
& =E\left(\sum_{i \in \mathcal{O}}\left\{\sum_{t=k}^{T} \operatorname{tr}\left[Q_{i}^{t} x(t) x(t)^{\prime}\right] \mathbb{1}_{\theta(t)=i}\right\}\right) \\
& =\sum_{t=k}^{T} \sum_{i \in \mathcal{O}}\left\{\operatorname{tr}\left[Q_{i}^{t} E\left(x(t) x(t)^{\prime} \mathbb{1}_{\theta(t)=i}\right)\right]\right\} .
\end{aligned}
$$

Agora, lembrando as definições (2.5) e (2.2), temos que

$$
\breve{\mathcal{W}}_{k}^{T}(i, x)=\sum_{t=k}^{T} \sum_{i \in \mathcal{O}}\left\{\operatorname{tr}\left[Q_{i}^{t} \breve{\mathcal{X}}_{i}^{t}\right]\right\}=\sum_{t=k}^{T}\left\langle Q^{t}, \breve{\mathcal{X}}^{t}\right\rangle
$$

o que verifica a parte i). Prosseguindo, utilizando o resultado (ii) da Proposição (2.3.1), podemos escrever

$$
\breve{\mathcal{W}}_{k}^{T}(i, x)=\left\langle Q^{k}, \breve{\mathcal{X}}^{k}\right\rangle+\sum_{t=k+1}^{T}\left\langle Q^{t}, \mathcal{T}^{t-1, k}\left(\breve{\mathcal{X}}^{k}\right)\right\rangle
$$

Empregando a dualidade entre os operadores $\mathcal{T}^{t}$ e $\mathcal{L}^{t}[10$, Proposição 3.2] à equação anterior é possível obter

$$
\breve{\mathcal{W}}_{k}^{T}(i, x)=\left\langle Q^{k}, \breve{\mathcal{X}}^{k}\right\rangle+\sum_{t=k+1}^{T}\left\langle\mathcal{L}^{k, t-1}\left(Q^{t}\right), \breve{\mathcal{X}}^{k}\right\rangle=\left\langle Q^{k}+\sum_{t=k+1}^{T} \mathcal{L}^{k, t-1}\left(Q^{t}\right), \breve{\mathcal{X}}^{k}\right\rangle
$$


Aplicando recursivamente a definição de $L^{t}$ é possível verificar que, para qualquer $k \leq \bar{k} \leq T-1$, é válido que $L^{\bar{k}}=Q^{\bar{k}}+\sum_{t=\bar{k}+1}^{T} \mathcal{L}^{\bar{k}, t-1}\left(Q^{t}\right)$. Portanto, particularizando a equação anterior para $\bar{k}=k$ e observando (A.10), é evidente que $\breve{\mathcal{W}}_{k}^{T}(i, x)=\left\langle L^{k}, \breve{\mathcal{X}}^{k}\right\rangle$. 
Apêndice

$B$

\section{Planejamento Hidrotérmico}

\section{B.1 Construção do Modelo}

Considere a equação de balanço hídrico para um reservatório,

$$
[x(t+1)]_{i}=[x(t)]_{i}-[r(t)]_{i}+\sum_{j \in \Omega_{i}}[r(t)]_{j}+[v(t)]_{i}-[l(t)]_{i}
$$

com $x_{i}(0)=x_{0, i}$; sendo $x_{i}(t)$ o volume armazenado de água no reservatório $i$ no início do $t$-ésimo intervalo de tempo, $r_{i}(t)$ o volume deplecionado (soma do volume vertido e turbinado) para o mesmo reservatório durante o intervalo $t, \Omega_{i}$ o conjunto de usinas imediatamente à montante do reservatório $i$, ou seja, cujos volumes deplecionados seguem diretamente para o reservatório $i, v_{i}(t)$ o volume incremental de água em cada reservatório e, por fim, $l_{i}(t)$ o volume de água perdido por evaporação. Com isto, para a usina de Sobradinho podemos escrever

$$
[x(t+1)]_{1}=[x(t)]_{1}-[r(t)]_{1}+[v(t)]_{1}-c_{e} h_{1}(t)\left[\frac{a_{1}\left([x(t)]_{1}\right)+a_{1}\left([x(t+1)]_{1}\right)}{2}\right] .
$$

Assumindo um modelo markoviano para a vazão afluente e uma relação linear entre a área e o volume do reservatório, expressa por $a_{1}\left([x(t)]_{1}\right)=a_{1}^{1}[x(t)]_{1}+a_{1}^{0}$, é possível 
obter

$$
\left(1+\frac{c_{e} h_{1}(t) a_{1}^{1}}{2}\right)[x(t+1)]_{1}=\left(1-\frac{c_{e} h_{1}(t) a_{1}^{1}}{2}\right)[x(t)]_{1}-[r(t)]_{1}+\left[v_{\theta(t)}(t)\right]_{1}-c_{e} h_{1}(t) a_{1}^{0} .
$$

Definindo $c_{m}^{1}(t)=\left(1+\left(c_{e} h_{1}(t) a_{1}^{1}\right) / 2\right)$ e $c_{m}^{2}(t)=\left(1-\left(c_{e} h_{1}(t) a_{1}^{1}\right) / 2\right)$ e utilizando $r(t)=$ $c_{v} u(t)+s(t)$ obtemos

$$
[x(t+1)]_{1}=\left(c_{m}^{1}(t)\right)^{-1}\left(c_{m}^{2}(t)[x(t)]_{1}-c_{v}[u(t)]_{1}-[s(t)]_{1}+\left(\left[v_{\theta(t)}(t)\right]_{1}-c_{e} h_{1}(t) a_{1}^{0}\right)\right) .
$$

Para Itaparica, podemos partir de B.1 e obter

$$
\begin{aligned}
{[x(t+1)]_{2}=\left(d_{m}^{1}(t)\right)^{-1} } & \left(d_{m}^{2}(t)[x(t)]_{2}-c_{v}[u(t)]_{2}-[s(t)]_{2}\right. \\
+ & \left.c_{v}[u(t)]_{1}+[s(t)]_{1}+\left([v(t)]_{2}-c_{e} h_{2}(t) a_{2}^{0}\right)\right)
\end{aligned}
$$

em que $d_{m}^{1}(t)=\left(1+\left(c_{e} h_{2}(t) a_{2}^{1}\right) / 2\right)$ e $d_{m}^{2}(t)=\left(1-\left(c_{e} h_{2}(t) a_{2}^{1}\right) / 2\right)$. Assim, definindo agora

$$
\begin{gathered}
A(t)=\left[\begin{array}{cc}
\left(c_{m}^{1}(t)\right)^{-1} c_{m}^{2}(t) & 0 \\
0 & \left(d_{m}^{1}(t)\right)^{-1} d_{m}^{2}(t)
\end{array}\right], \\
B(t)=\left[\begin{array}{cc}
-c_{v}\left(c_{m}^{1}(t)\right)^{-1} & 0 \\
c_{v}\left(d_{m}^{1}(t)\right)^{-1} & -c_{v}\left(d_{m}^{1}(t)\right)^{-1}
\end{array}\right], \\
e_{\theta(t)}(t)=\left[\begin{array}{c}
\left(c_{m}^{1}(t)\right)^{-1}\left(\left[v_{\theta(t)}(t)\right]_{1}-c_{e} h_{1}(t) a_{1}^{0}\right) \\
\left(d_{m}^{1}(t)\right)^{-1}\left([v(t)]_{2}-c_{e} h_{2}(t) a_{2}^{0}\right)
\end{array}\right] \mathrm{e} \\
C(t)=\left[\begin{array}{cc}
-\left(c_{m}^{1}(t)\right)^{-1} & 0 \\
\left(d_{m}^{1}(t)\right)^{-1} & -\left(d_{m}^{1}(t)\right)^{-1}
\end{array}\right],
\end{gathered}
$$

obtemos o modelo

$$
x(t+1)=A(t) x(t)+B(t) u(t)+e_{\theta(t)}(t)+C(t) s(t) .
$$

Utilizando os valores da Tabela B.3, $c_{v}=2.592$ e $c_{e}=10^{-3}$, obtivemos os seguintes valores para as matrizes $A(t)$ e $B(t)$ referentes ao cenário 1. É interessante observar que para o cenário 2 as matrizes $A(t)$ se reduzem à matriz identidade, e $B(t)$ e $C(t)$ passam a ser invariantes no tempo. 
Tabela B.1: Elementos correspondentes às matrizes $A(t)$ e $B(t)$.

$\begin{array}{lcccccc} & \text { Jan } & \text { Mar } & \text { Mai } & \text { Jul } & \text { Set } & \text { Nov } \\ {[A(t)]_{1,1}=} & 0.9821 & 0.9936 & 0.9887 & 0.9828 & 0.9756 & 0.9745 \\ {[A(t)]_{2,2}=} & 0.9905 & 0.9972 & 0.9968 & 0.9952 & 0.9889 & 0.9863 \\ {[B(t)]_{1,1}=} & -2.5689 & -2.5837 & -2.5773 & -2.5697 & -2.5604 & -2.5590 \\ {[B(t)]_{2,2}=} & 2.5796 & 2.5884 & 2.5878 & 2.5858 & 2.5776 & 2.5742\end{array}$

\section{B.2 Dados dos Reservatórios}

Tabela B.2: Principais características dos reservatórios estudados.

$\begin{array}{lcccc} & \begin{array}{c}\text { Protutibilidade } \\ \text { Específica } \\ \left(M W / \mathrm{m}^{2} \mathrm{~s}^{-1}\right)\end{array} & \begin{array}{c}\text { Engolimento } \\ \text { máximo } \\ \left(\mathrm{m}^{3} / \mathrm{s}\right)\end{array} & \begin{array}{c}\text { Volume } \\ \text { Máximo } \\ \left(\mathrm{hm}^{3}\right)\end{array} & \begin{array}{c}\text { Volume } \\ \text { Mínimo } \\ \left(\mathrm{hm}^{3}\right)\end{array} \\ \text { Sobradinho } & 0.009023 & 4278 & 5447 & 34116 \\ \text { Itaparica } & 0.008931 & 3306 & 7234 & 10782 \\ \text { C. Moxotó } & 0.009035 & 4201 & 1226 & 1226 \\ \text { Xingó } & 0.009119 & 2976 & 3800 & 3800\end{array}$

Tabela B.3: Médias mensais da taxa de evaporação líquida $\left(h_{i}(t)\right)$ em $m m / m e ̂ s$.

$\begin{array}{lcccccccccccc} & \text { Jan } & \text { Fev } & \text { Mar } & \text { Abr } & \text { Mai } & \text { Jun } & \text { Jul } & \text { Ago } & \text { Set } & \text { Out } & \text { Nov } & \text { Dez } \\ \text { Sobradinho } & 171 & 109 & 61 & 56 & 108 & 104 & 165 & 203 & 234 & 267 & 245 & 223 \\ \text { Itaparica } & 163 & 88 & 47 & 35 & 55 & 41 & 81 & 138 & 190 & 227 & 235 & 202 \\ \text { C. Moxotó } & 163 & 88 & 47 & 35 & 55 & 41 & 81 & 138 & 190 & 227 & 235 & 202 \\ \text { Xingó } & 163 & 88 & 47 & 35 & 55 & 41 & 81 & 138 & 190 & 227 & 235 & 202\end{array}$

Tabela B.4: Coeficientes do polinômio cota vs volume $\left(\varphi_{i}().\right)$.

$\begin{array}{lccccc} & \varphi_{0} & \varphi_{1} & \varphi_{2} & \varphi_{3} & \varphi_{4} \\ \text { Sobradinho } & 3.741790 \mathrm{E} 2 & 1.396690 \mathrm{E}-3 & -5.351590 \mathrm{E}-8 & 1.155989 \mathrm{E}-12 & -9.545989 \mathrm{E}-18 \\ \text { Itaparica } & 2.758130 \mathrm{E} 2 & 6.764889 \mathrm{E}-3 & -8.868370 \mathrm{E}-7 & 7.067990 \mathrm{E}-11 & -2.239850 \mathrm{E}-15 \\ \text { C. Moxotó } & 2.515000 \mathrm{E} 2 & 0 & 0 & 0 & 0 \\ \text { Xingó } & 1.380000 \mathrm{E} 2 & 0 & 0 & 0 & 0\end{array}$


Tabela B.5: Coeficientes do polinômio cota vs vazão defluente $\left(\mu_{i}().\right)$.

$\begin{array}{lccccc} & \mu_{0} & \mu_{1} & \mu_{2} & \mu_{3} & \mu_{4} \\ \text { Sobradinho } & 3.596538 \mathrm{E} 2 & 1.964010 \mathrm{E}-3 & -2.968730 \mathrm{E}-7 & 2.508280 \mathrm{E}-11 & -7.702299 \mathrm{E}-16 \\ \text { Itaparica } & 2.515000 \mathrm{E} 2 & 0 & 0 & 0 & 0 \\ \text { C. Moxotó } & 1.380000 \mathrm{E} 2 & 0 & 0 & 0 & 0 \\ \text { Xingó } & 1.003861 \mathrm{E} 1 & 6.665461 \mathrm{E}-3 & -2.454770 \mathrm{E}-6 & 4.559482 \mathrm{E}-10 & -3.144850 \mathrm{E}-14\end{array}$

Tabela B.6: Coeficientes do polinômio área vs cota $\left(\rho_{i}().\right)$.

$\begin{array}{lccccc} & \rho_{0} & \rho_{1} & \rho_{2} & \rho_{3} & \rho_{4} \\ \text { Sobradinho } & -5.037100 \mathrm{E} 5 & 4.913789 \mathrm{E} 3 & -8.966889 \mathrm{E} 0 & -1.891690 \mathrm{E}-2 & 4.653790 \mathrm{E}-5 \\ \text { Itaparica } & -1.996950 \mathrm{E} 5 & 1.822240 \mathrm{E} 3 & -4.435699 \mathrm{E} 0 & -1.917610 \mathrm{E}-3 & 1.292100 \mathrm{E}-5 \\ \text { C. Moxotó } & 2.130000 \mathrm{E} 0 & 0 & 0 & 0 & 0 \\ \text { Xingó } & 6.000000 \mathrm{E} 1 & 0 & 0 & 0 & 0\end{array}$




\section{B.3 Modelo Markoviano para a Vazão Afluente de So- bradinho}

A Figura B.1 apresenta a série real de vazão afluente para o reservatório de Sobradinho. Já a Figura B.2, apresenta os valores característicos atribuídos a cada modo da cadeia $\mathbb{P}_{v}(t)$, com a utilizações do método disposto em [17]. Na sequência apresentamos ainda a cadeia $\mathbb{P}_{v}(t)$ obtida para alguns meses do ano.

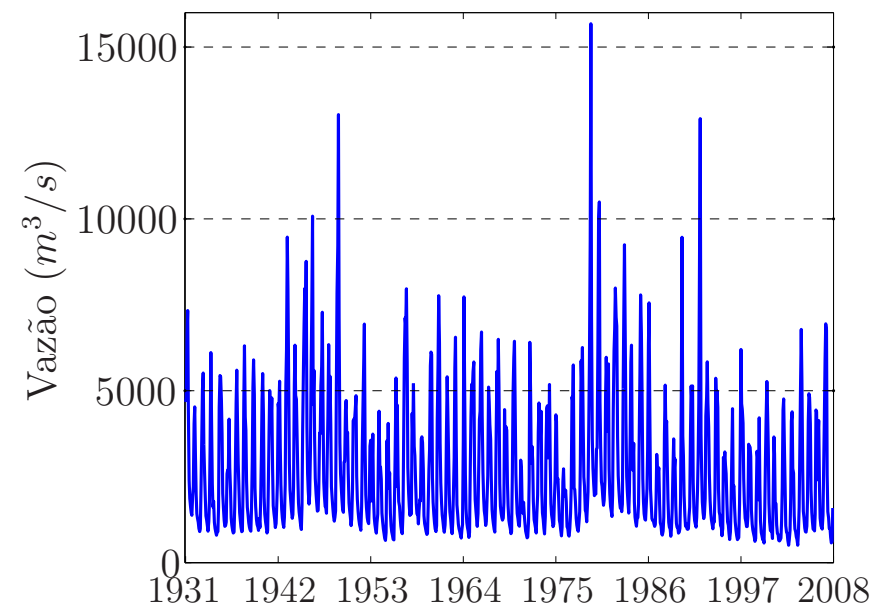

Figura B.1: Série histórica da vazão afluente de Sobradinho.

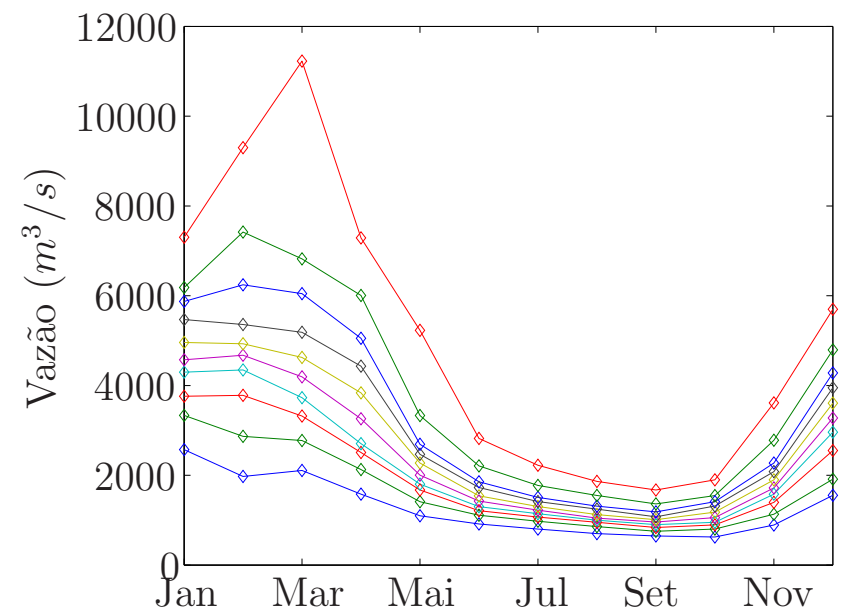

Figura B.2: Vazões representativas dos estados de $\mathbb{P}_{v}(t)$. 


\section{Janeiro}

$$
\mathbb{P}_{v}(9)=\left[\begin{array}{cccccccccc}
0.375 & 0.25 & 0.25 & 0 & 0.125 & 0 & 0 & 0 & 0 & 0 \\
0.25 & 0.125 & 0.125 & 0 & 0.125 & 0.125 & 0 & 0.125 & 0.125 & 0 \\
0 & 0.125 & 0.125 & 0.125 & 0.125 & 0.125 & 0.375 & 0 & 0 & 0 \\
0.125 & 0.125 & 0.125 & 0.125 & 0 & 0.25 & 0.125 & 0.125 & 0 & 0 \\
0.125 & 0.125 & 0.125 & 0.125 & 0.125 & 0 & 0.25 & 0 & 0 & 0.125 \\
0 & 0.125 & 0 & 0.125 & 0.125 & 0.125 & 0.125 & 0.25 & 0.125 & 0 \\
0.125 & 0 & 0.125 & 0.125 & 0.125 & 0.125 & 0 & 0.25 & 0.125 & 0 \\
0 & 0 & 0 & 0 & 0.143 & 0 & 0.143 & 0.143 & 0.429 & 0.143 \\
0 & 0.125 & 0.125 & 0.25 & 0.125 & 0.25 & 0 & 0 & 0 & 0.125 \\
0 & 0 & 0 & 0.167 & 0 & 0 & 0 & 0 & 0.167 & 0.667
\end{array}\right]
$$

Maio

$$
\mathbb{P}_{v}(1)=\left[\begin{array}{cccccccccc}
0.875 & 0.125 & 0 & 0 & 0 & 0 & 0 & 0 & 0 & 0 \\
0.125 & 0.5 & 0.125 & 0.25 & 0 & 0 & 0 & 0 & 0 & 0 \\
0 & 0.25 & 0.5 & 0.125 & 0.125 & 0 & 0 & 0 & 0 & 0 \\
0 & 0.125 & 0.25 & 0.25 & 0.25 & 0.125 & 0 & 0 & 0 & 0 \\
0 & 0 & 0.125 & 0.25 & 0.5 & 0.125 & 0 & 0 & 0 & 0 \\
0 & 0 & 0 & 0.125 & 0.125 & 0.5 & 0.125 & 0.125 & 0 & 0 \\
0 & 0 & 0 & 0 & 0 & 0.125 & 0.5 & 0.25 & 0.125 & 0 \\
0 & 0 & 0 & 0 & 0 & 0.143 & 0.286 & 0.429 & 0.143 & 0 \\
0 & 0 & 0 & 0 & 0 & 0 & 0.143 & 0.143 & 0.286 & 0.429 \\
0 & 0 & 0 & 0 & 0 & 0 & 0 & 0 & 0.429 & 0.571
\end{array}\right]
$$

Setembro

$$
\mathbb{P}_{v}(5)=\left[\begin{array}{cccccccccc}
0.625 & 0.25 & 0.125 & 0 & 0 & 0 & 0 & 0 & 0 & 0 \\
0.25 & 0.25 & 0 & 0.25 & 0.125 & 0.125 & 0 & 0 & 0 & 0 \\
0 & 0.25 & 0.25 & 0.125 & 0 & 0.25 & 0.125 & 0 & 0 & 0 \\
0 & 0.125 & 0.125 & 0.25 & 0.125 & 0.25 & 0 & 0.125 & 0 & 0 \\
0.125 & 0 & 0.375 & 0.125 & 0 & 0.125 & 0.125 & 0.125 & 0 & 0 \\
0 & 0.125 & 0.125 & 0.125 & 0.125 & 0.125 & 0.25 & 0.125 & 0 & 0 \\
0 & 0 & 0 & 0.125 & 0.375 & 0.125 & 0.125 & 0.125 & 0.125 & 0 \\
0 & 0 & 0 & 0 & 0.286 & 0 & 0.286 & 0 & 0.286 & 0.143 \\
0 & 0 & 0 & 0 & 0 & 0 & 0.143 & 0.286 & 0.286 & 0.286 \\
0 & 0 & 0 & 0 & 0 & 0 & 0 & 0.143 & 0.429 & 0.429
\end{array}\right]
$$




\section{B.4 Demais Resultados - Figuras}

Na Figura B.3 apresentamos o custo esperado e a covariância obtidos pelo RLQ em relação à PDD para o cenário 2. Estes valores estão apresentados de acordo $S$, definido como o número de realizações obtido com a simulação de Monte Carlo.

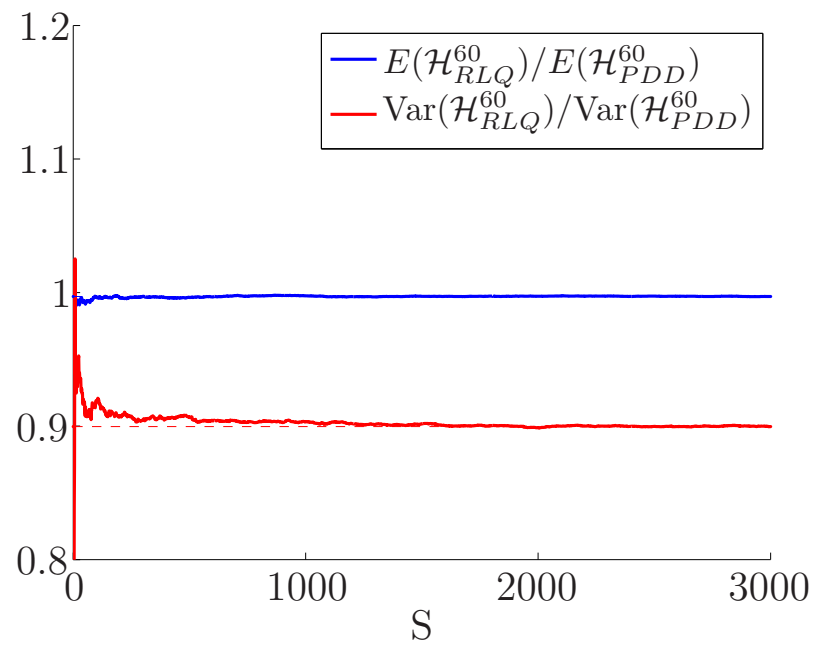

Figura B.3: Convergência do valor esperado e covariância do custo para o cenário 2.

Nas figuras B.4 e B.5 apresentamos as trajetórias esperadas para a turbinagem do reservatório de Sobradinho para o horizonte de 5 anos. Já nas figuras B.6 e B.7 apresentamos as trajetórias esperadas para o volume do reservatório de Itaparica. Na sequência, figuras B.8 e B.10, apresentamos os valores esperados para a turbinagem de Itaparica. Note que a turbinagem desta usina têm uma estreita relação com custo, figuras B.9 e B.11, respectivamente. As linhas verdes indicam as restrições inferiores e superiores para a variável considerada e a curva tracejada corresponde à curva guia obtida para o RLQ. Para os gráficos que expõem a turbinagem, as linhas na parte inferior indicam o vertimento esperado para cada mês. 


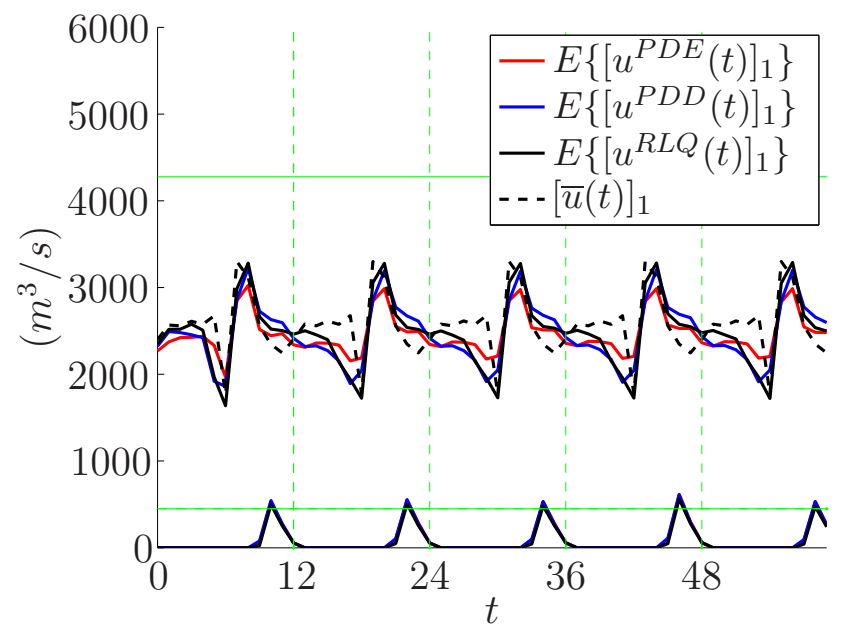

Figura B.4: Turbinagem esperada para Sobradinho no cenário 1.

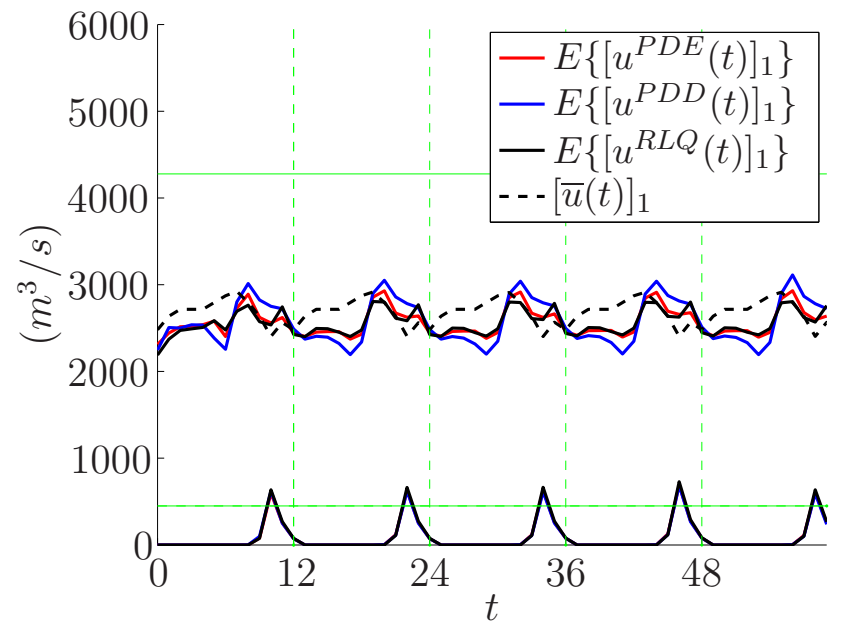

Figura B.5: Turbinagem esperada para Sobradinho no cenário 2. 


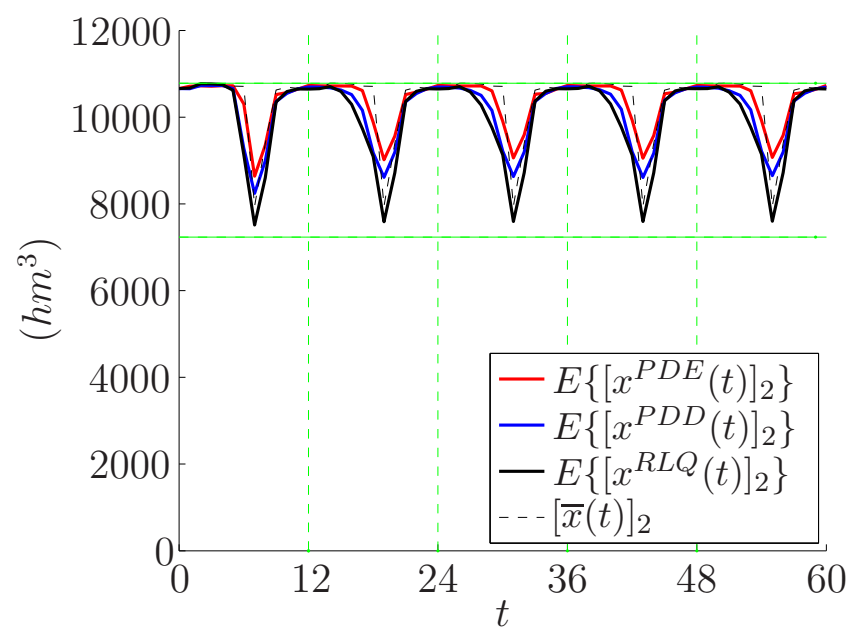

Figura B.6: Volume esperado para Itaparica no cenário 1.

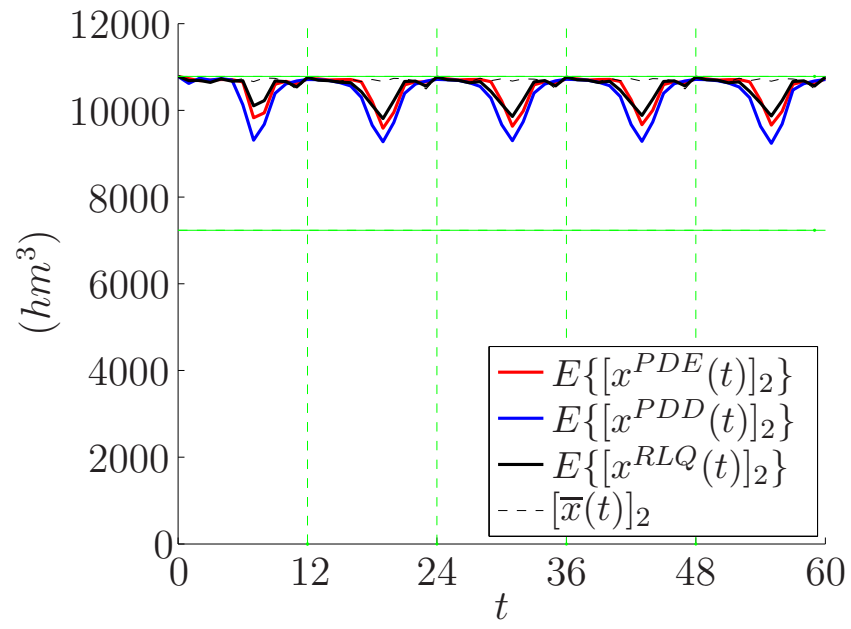

Figura B.7: Volume esperado para Itaparica no cenário 2. 


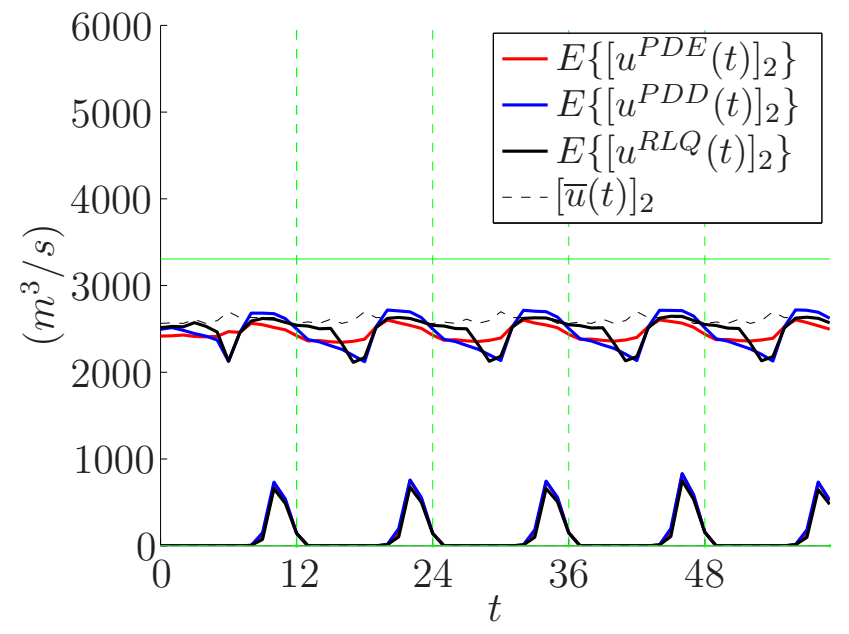

Figura B.8: Turbinagem esperada para Itaparica no cenário 1.

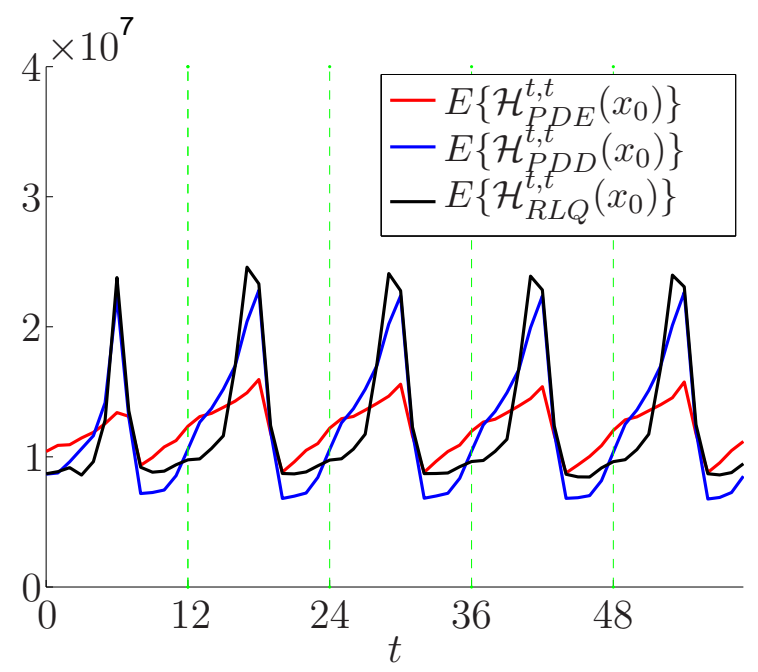

Figura B.9: Custo mensal esperado para o cenário 1. 


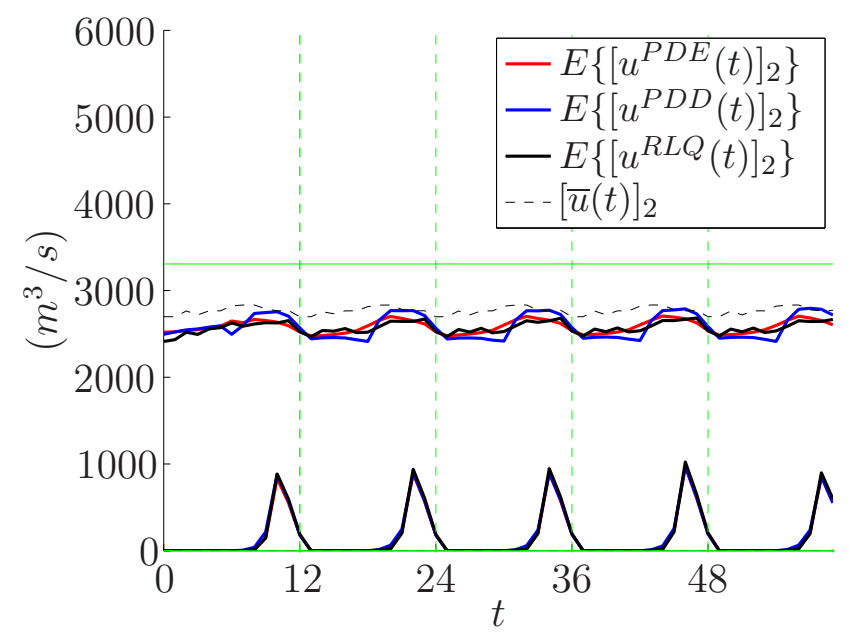

Figura B.10: Turbinagem esperada para Itaparica no cenário 2.

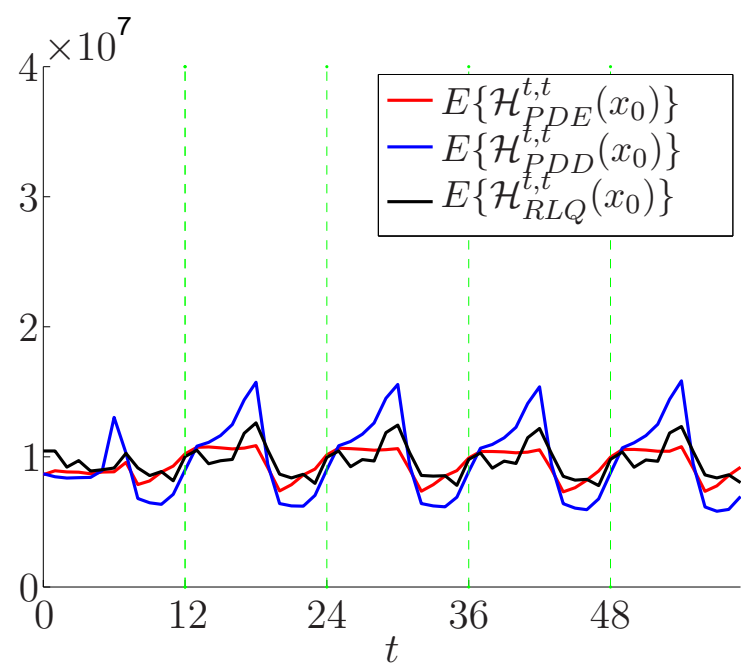

Figura B.11: Custo mensal esperado para o cenário 2. 